\title{
TRANSPORT \\ A COMPUTER PROGRAM FOR DESIGNING CHARGED PARTICLE BEAM TRANSPORT SYSTEMS
}

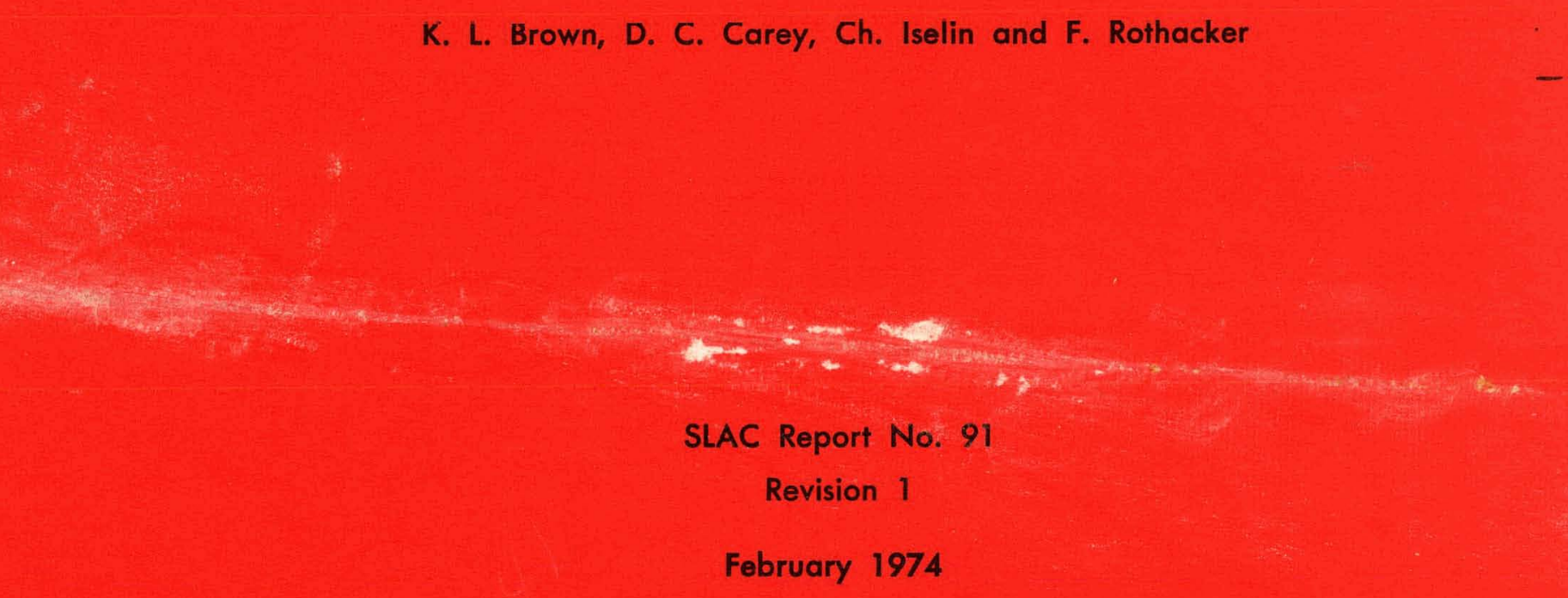

Under Contract with the U. S. Atomic Energy Commission Contract AT(04-3)-515

\section{MASTER}




\section{DISCLAIMER}

This report was prepared as an account of work sponsored by an agency of the United States Government. Neither the United States Government nor any agency Thereof, nor any of their employees, makes any warranty, express or implied, or assumes any legal liability or responsibility for the accuracy, completeness, or usefulness of any information, apparatus, product, or process disclosed, or represents that its use would not infringe privately owned rights. Reference herein to any specific commercial product, process, or service by trade name, trademark, manufacturer, or otherwise does not necessarily constitute or imply its endorsement, recommendation, or favoring by the United States Government or any agency thereof. The views and opinions of authors expressed herein do not necessarily state or reflect those of the United States Government or any agency thereof. 


\section{DISCLAIMER}

Portions of this document may be illegible in electronic image products. Images are produced from the best available original document. 
This report was prepared as an account of work sponsored by the United States Government. Neither the United States
nor the United States Atomic Energy Commission, nor any of their employees, nor any of their contractors, subcontractors, or their employees, makes any warranty, express or implied, or assumes any legal liability or responsibility for the accuracy, completeness or usefulness of any informa ion, apparatus, product or process disclosed, or represents that its use would not infringe privately-owned rights. 


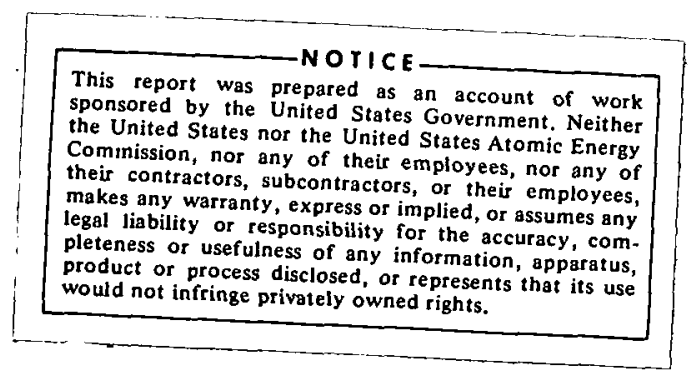

\section{TRANSPORT}

A COMPUTER PROGRAM FOR DESIGNING

CHARGED ṔARTICLE BEAM TRANSPORT SYSTEMS*

K. L. Brown and F. Rothacker

Stanford Linear Accelerator Center, Stanford, California 94305

D. C. Carey

National Accelerator Laboratory, Batavia, Mlinois 60510

Ch. Iselin

CERN, Geneva, Switzerland

PREPARED FOR THE U.S. ATOMIC ENERGY

COMMISSION UNDER CONTRACT AT(04-3) -515

February 1974

Printed in the United States of America. Available from National Technical Information Service, U.S. Department of Commerce, 5285 Port Royal Road, Springfield, Virginia 22151.

Price: $\$ 5.45$; Microfiche $\$ 0.95$.

* This report also is published as CERN-73-16 and NAL-91.

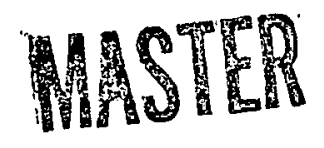


THIS PAGE

WAS INTENTIONALLY

LEFT BLANK 


\section{CONTENTS}

\section{Page}

INTRODUCTION

MATHEMATICAL FORMULATION OF "TRANSPORT"

General conventions 3

The transfer matrix $R \quad 4$

The beam matrix $\sigma \quad 6$

Fitting $\quad 8$

$\begin{array}{ll}\text { INPUT FORMAT FOR TRANSPORT } & 10\end{array}$

An Example of a TRANSPORT Input Deck 14

The use of labels $\quad 15$

$\begin{array}{lll}\text { Table 1: } & \text { Summary of TRANSPORT type codes } & 18\end{array}$

$\begin{array}{ll}\text { OUTPUT FORMAT } & 19\end{array}$

General appearance $\quad 19$

Initial listing 19

Listing during the calculation $\quad 20$

$\begin{array}{ll}\text { Calculated quantities } & 20\end{array}$

$\begin{array}{ll}\text { TITLE } \cdot \text { CARD } & 28\end{array}$

INDICATOR C.ARD $(0$ or \pm 1$) \quad . \quad 29$

$\begin{array}{ll}\text { COMMENT CARDS } & 30\end{array}$

Example of the use of comment cards in a data set 30

LISTING OF AVAILABLE TRANSPORT TYPE CODE ENTRIES _ 31

INPUT BEAM: Type code $1.0 \quad 31$

The phase ellipse beam matrix 33

r.m.s. addition to the BEAM 36

FRINGING FIELDS and POLE-FACE ROTATIONS for bending magnets:

Type code 2.0 
Pole-face rotation matrix

DRIFT: Type code 3.0

DRIET space matrix

WEDGE BENDING MAGNET: Type code 4.0

First-order wedge bending magnet matrix

QUADRUPOLE: Type code 5.0

First-order quadmuole matrix

TRANSFORM 1 update: Type code 6. 0. 1 .

AUXILIARY TRANSFORMATION MATRIX (R2): Type code 6. 0. 2 .

MAGNET ALIGNMENT TOLERANCES: Type code 8.0 .

Excomple No. 1: A misaligned bending magnet

Example No. 2: A misaligned bending magnet

Exomple No. 3: A misaligned quadmopolo triplat

REPETITION: Type code 9.0

Example of Nesting

VARY CODES and FITTING CONSTRAINTS: Type code 10.0

Vary codes

First-urder vary codes

Second-order vary codes

coupled vary codes 66

Poceiblo fitting conotrainto

First-order constraints

Second-order constraints 
R2 matrix fitting constraints 70

$\sigma$ (BEAM) matrix fitting constraints 71

Beam correlation matrix ( $r$ ) fitting constraints 72

First moment constraint : 73

$\begin{array}{ll}\text { System length constraint } & 74\end{array}$

AGS machine constraint $\quad 75$

$\begin{array}{ll}\text { T1 matrix fitting constraints } & 76\end{array}$

T2 matrix fitting constraints 77

Second-order $\sigma$ (BEAM) matrix fitting constraint 78

Sextupole strength constraints $\quad 79$

Internal constraints $\quad 80$

Corrections and covariance matrix 81

ACCELERATION: $\quad$ Type code $11.0 \quad 82$

Accelerator section matrix

BEAM (rotated ellipse): $\quad$ Type code $12.0 \quad-84$

Output PRINT CONTROL instructions: Type code $13.0 \quad 85$

Printed oitput controls 1., 2., 3., 4., 24. 85

Coordinate layout control 12

Punched output controls 29, 30, 31, 32, 33, 34, 35, 36. 86

ARBITRARY TRANSFORMATION input: Type code $14.0 \quad 88$

$\begin{array}{ll}\text { Input-output UNITS: } & 90\end{array}$

SPECIAL INPUT PARAMETERS: $\quad$ Type code $16.0 \quad 94$

Code digits for special parameters $\quad 94$

Tilt-to-focal plane (16. 15. a. ;) element 98

Initial heam line conodinates and direction 100

SECOND-ORDER CALCULATION: Type code $17.0 \quad 102$

IEXTUPOLE: Type code $18.0 \quad 103$ 
SOLENOID: Type code 19.0 105

First-order solenoid matrix 106

COORDINATE ROTATION: Type code 20.0 108

Examp Zes 109

STRAY MAGNETIC FIELD: Type code 21.0 111

SENTINEL

REFERENCES 


\section{INTRODUCTION}

TRANSPORT is a first- and second-order matrix multiplication computer program intended for the design of static-magnetic beam transport systems. It has been in existance in various evolutionary versions since 1963. The present version, described in this manual, includes both first- and secondorder fitting capabilities.

Many people from various laboratories around the world have contributed either directly or indirectly to the development of TRANSPORT. The first-order matrix methods were developed by the AGS machine theorists ${ }^{1}$ ) followed by a paper by Penner ${ }^{2}$. The extension of the first-order matrix methods to include second and higher orders was conceived and developed by Brown, Belbeoch and Bounin ${ }^{3)}$ in Orsay, France, in 1958-59. The original first-order TRANSPORT computer program was written in BALGOL by C.H. Moore at SLAC in collaboration with H.S. Butler and S.K. Howry in 1963. The second-order portion of the program was developed and debugged by Howry and Brown ${ }^{4}$, also in BALGOL. The resulting BALGOL version was translated into FORTRAN by $\mathrm{S}$. Kowalski at MIT and later debugged and improved by Kear, Howry and Brown at SLAC $^{5)}$. In 1971-72, D. Carey at NAL completely rewrote the program and developed an efficient secondorder fitting routine using the coupling coefficients (partial derivatives) of multipole components to the optics as derived by Brown ${ }^{6}$ ). This version was implemented at SLAC by F. Rothacker in the early spring of 1972 and subsequently carried to CERN in Apri1, 1972, by K.L. Brown. C. Iselin of CERN made further contributions to the program structure and improved the convergence capabilities of the first-order fitting routines.

A standard version of the resulting program has now been adopted at SLAC, NAL, and CERN. This manual describes the use of this standard version and is not necessarily applicable to other versions of TRANSPORT. Copico of thic manual may also be obtained from

1) The Reports Office, CERN, 23 Geneva, Switzerland (Ref. CERN 73-16).

2) The Reports Office, National Accelerator Laboratory, P.0. Box 500, Ba.tavia, ПI. 60510 (Ref. NAL-91). 
A standard version of TRANSPORT has been implemented on the IBM 360, using the FORTRAN $H$ compiler. It requires approximately 270,000 bytes. of memory for operation. It is normally distributed on 9 track magnetic tape at 800 BPI having a standard IBM label. This program may be obtained from

TRANSPORT Program Librarian

Stanford Linear Accelerator Center

P. O. Box 4349

Stanford, CA 94305

CDC 7600 users are encouraged to obtain the program from .

Ch. Iselin

$\mathrm{CH}-1211$

CERN

23 Geneva, Switzerland

PDP 10 users should contact

D. C. Carey

Research Services

National Accelerator Laboratory

P. 0. Box 500

Batavia, II 60510

The present authors assume responsibility for the contents of this manual, but in no way imply that they are solely responsible for the entire evolution of the program. 
MATHEMATICAL FORMULATION OF "TRANSPORT" *)

General conventions

A beam line is comprised of a set of magnetic elements placed sequentially at intervals along an assumed reference trajectory. The reference trajectory is here taken to be a path of a charged particle passing through idealized magnets (no fringing fields) and having the central design momentum of the beam line.

In TRANSPORT, a beam line is described as a sequence of elements. Such elements may consist not only of magnets and the intervals between them, but also of specifications of the input beam, calculations to be done, or special configurations of the magnets. A certain relation, described below, of the magnets and their fields to the assumed reference trajectory is considered normal. Alternative configurations can be described by means of elements provided for such purposes.

The two coordinates transverse to the initial reference trajectory are labelled as horizontal and vertical. A bending magnet will normally bend in the horizontal plane. To allow for other possibilities a coordinate rotation element is provided. Because of such other possibilities, when describing bending magnets we shall often speak of the bend and nonbend planes. The transverse coordinates will also often be labelled $x$ and $y$, while the longitudinal coordinate will be labelled $z$.

A11 magnets are normally considered "aligned" on the central trajectory. A particle following the central trajectory through a magnet experiences a uniform field which begins and ends abruptly at the entrance and exit faces of the idealized magnet. Therefore, through a bending magnet the reference trajectory is the arc of a circle, while through all other magnetic elements it is a straight line. To accommodate a more gradual variation of field at the ends of a bending magnet a fringing field element is provided. In order to represent an orientation with respect to the reference trajectory other than normal of a magnet or section of a beam line, a misalignment element also exists.

The magnetic field of any magnet, except a solenoid, is assumed to have midplane symmetry. This means that the scalar potential expanded

*) For a more complete description of the mathematical basis of TRANSPORT, refer to SLAC-75 4 ), and to other references listed at the end of this manual. 
in transverse coordinates about the reference trajectory is taken to be an odd function of the vertical coordinate. If a coordinate rotation is included, then the potential is odd in the coordinate to which the vertical has been rotated. For a bending magnet this will always be in the non-bend plane.

The program TRANSPORT will step through the beam line, element by element, calculating the properties of the beam or other quantities, described below, where requested. Therefore one of the first elements is a specification of the phase space region occupied by the beam entering the system. Magnets and intervening spaces and other elements then follow in the sequence in which they occur in the beam line. Specifications of calculations to be done or of configurations other than normal are placed in the same sequence, at the point where their effect is to be made.

The transfer matrix $R$

The following of a charged particle through a system of magnetic lenses may be reduced to a process of matrix multiplication. At any specified position in the system an arbitrary charged particle is represented by a vector (single column matrix) $\mathrm{X}$, whose components are the positions, angles, and momentum of the particle with respect to the reference trajectory, i.e.

$$
\mathrm{X}=\left[\begin{array}{c}
\mathrm{x} \\
\theta \\
\mathrm{y} \\
\phi \\
\ell \\
\delta
\end{array}\right]
$$

Definitions:

$x=$ the horizontal displacement of the arbitrary ray with respect to the assumed central trajectory.

$\theta=$ the angle this ray makes in the horizontal plane with respect to the assumed central trajectory.

$y=$ the vertical displacement of the ray with respect to the assumed central trajectory.

$\phi=$ the vertical angle of the ray with respect to the assumed central trajectory. 
$\ell=$ the path length difference between the arbitrary ray and the central trajectory.

$\delta=\Delta \mathrm{p} / \mathrm{p}$ is the fractional momentum deviation of the ray from the assumed central trajectory.

This vector, for a given particle, will henceforth be referred to as a ray. The magnetic lens is represented to first order by a square matrix $R$, which describes the action of the magnet on the particle coordinates. Thus the passage of a charged particle through the system may be represented by the equation

$$
X(1)=\operatorname{RX}(0),
$$

where $X(0)$ is the initial coordinate vector and $X(1)$ is the final coordinate vector of the particle under consideration. The same transformation matrix $R$ is used for all such particles traversing a given magnet [one particle differing from another only by its initial coordinate vector $x(0)]$.

The traversing of several magnets and interspersing drift spaces is described by the same basic equation, but with $R$ now being replaced by the product matrix $R(t)=R(n) \ldots R(3) R(2) R(1)$ of the individual matrices of the system elements. This cumulative transfer matrix is automatically calculated by the program and is called TRANSFORM 1. It may be printed where desired, as described in later sections.

This formalism may be extended to second order by the addition of another term ${ }^{4}$ ). The components of the final coordinate vector, in terms of the original, are now given as

$$
x_{i}(1)=\sum_{j} R_{i j} x_{j}(0)+\sum_{j k} T_{i j k} x_{j}(0) x_{k}(0),
$$

where $T$ is the second-nrder transfer matrix, It too is accumulated by the program as one traverses a series of elements. At each point the series is again truncated to second order. Normally the program will calculate only the first-order terms and their effect. If it is desired to include second-order effects in a beam line, an element is provided which specifies that a second-order calculation is to be done. For more information on the $T$ matrix, see the references at the end of the manual. 
The following of a charged particle via TRANSPORT through a system of magnets is thus analogous to tracing rays through a system of optical lenses. The difference is that TRANSPORT is a matrix calculation which truncates the problem to either first- or second-order in a Taylor's expansion about a central trajectory. For studying beam optics to greater precision than a second-order TRANSPORT calculation permits, ray-tracing programs which directly integrate the basic differential equation of motion are recommended ${ }^{7)}$.

The beam matrix $\sigma$

Tn accelerator and beam transport systems, the behaviour of an individual particle is often of less concern than is the behaviour of a bundle of particles (the beam), of which an individual particle is a member. An extension of the matrix algebra of $\mathrm{Eq}$. (1) provides a convenient means for defining and manipulating this beam. TRANSPORT assumes that the beam may be correctly represented in phase space by an ellipsoid in the sixdimensional coordinate system described above. Particles in a beam are assumed to occupy the volume enclosed by the ellipsoid, each point representing a possible ray. The sum total of all phase points, the phase space volume, is commonly referred to as the "phase space" occupied by the beam.

The validity and interpretation of this phase ellipse formalism must be ascertained for each system being designed. However, in general, for charged particle beams in, or emanating from accelerators, the firstorder phase ellipse formalism of TRANSPORT is a reasonable representation of physical reality. For other applications, such as charged particle spectrometers, caution is in order in its use and interpretation.

The equation of an n-dimensional ellipsoid may be written in matrix form as follows:

$$
X(0)^{T} \sigma(0)^{-1} X(0)=1
$$

where $X(0)^{T}$ is the transpose of the coordinate vector $X(0)$, and $\sigma(0)$ is a real, positive definite, symmetric matrix.

The volume of the n-dimensional ellipsoid defined by sigma is $\left[\pi^{n / 2} / \Gamma(n / 2+1)\right](\operatorname{det} \sigma)^{\frac{1}{2}}$. The area of the projection in one plane is 
$A=\pi\left(\operatorname{det} \sigma_{1}\right)^{\frac{1}{2}}$, where $\sigma_{1}$ is the submatrix corresponding to the given plane. This is the "phase space" occupied by the beam.

As a particle passes through a system of magnets, it undergoes the matrix transformation of Eq. (1). Combining this transformation with the equation of the initial ellipsoid, and using the identity $R^{-1}=I$ (the unity matrix), it follows that

$$
X(0)^{T}\left(R^{T} R^{T^{-1}}\right) \sigma(0)^{-1}\left(R^{-1} R\right) X(0)=1
$$

from which we derive

$$
[\mathrm{RX}(0)]^{\mathrm{T}}\left[\mathrm{R} \sigma(0) \mathrm{R}^{\mathrm{T}}\right]^{-1}[\mathrm{RX}(0)]=1
$$

The equation of the new ellipsoid after the transformation becomes

$$
X(1)^{\mathrm{T}} \sigma(1)^{-1} \mathrm{X}(1)=1
$$

where

$$
\sigma(1)=R \sigma(0) R^{T}
$$

It can readily be shown that the square roots of the diagonal terms of the sigma matrix are a measure of the "beam size" in each coordinate. The off-diagonal terms determine the orientation of the ellipsoid in $\mathrm{n}$-dimensional space (for TRANSPORT $\mathrm{n}=6)^{*}$ ). Thus, we may specify the beam at any point in the system via Eq. (5), given the initial "phase space" represented by the matrix elements of $\sigma(U)$.

The initial beam is specified by the user as one of the first elements of the beam line. Normally it is taken to be an upright ellipse centred on the reference trajectory; that is, there are no correlations between coordinates. Both correlations and centroid displacements may be introduced via additional elements.

*) See the Appendix of this report (to be published under a separate cover), or the Appendix of Ref. 5, for a derivation of these statements. 
The phase ellipse is normally printed after each physical element. For an interpretation of the parameters printed see the section under type code 1.0 .

When a second-order calculation is specified the second-order matrix elements are included in the beam matrix. For details on how this is done see the Appendix to this manual.

\section{Fitting}

Several types of physical elements have been incorporated in the program to facilitate the design of very general beam transport systems. Included are an arbitrary drift distance, bending magnets, quadrupoles, sextupoles, solenoids, and an accelerator section (to first-order only). Provision is made in the program to vary some of the physical parameters of the elements comprising the system and to impose various constraints on the beam design. In a first-order run one may fit either the TRANSFORM ( $R$ ) matrix representing the transformation of an arbitrary ray through the system and/or the phase ellipse (sigma) matrix representing a bundle of rays by the system as transformed. In a second-order run one may fit either the second-order TRANSFORM ( T) matrix or minimize the net contribution of second-order terms to the beam (sigma) matrix.

The program will normally make a run through the beam line using values for the physical parameters as specified by the user and printing the results. If constraints and parameters to be varied are indicated, it will attempt to fit. To do this it wili make an additional series of runs through the beam line. Each time it will calculate corrections to be made from the previous step to the varied parameters to try to satisfy the indicated constraints. When the constraints are satisfied (or the fitting procedure has failed) the program will make a final run through the beam line again printing the results. In this final run the values of the physical parameters used are those which are the result of the fitting procedure.

Thus, in principle, the program is capable of searching for and finding the first- or second-order solution to any physically realizable problem. In practice, life is not quite so simple. The user will find that an adequate knowledge of geometric magnetic optics principles is a 
necessary prerequisite to the successful use of TRANSPORT. He (or she) should possess a thorough understanding of the first-order matrix algebra of beam transport optics and of the physical interpretation of the various matrix elements.

In other words, the program is superb at doing the numerical calculations for the problem but not the physics. The user must provide a reasonable physical input if he (or she) expects complete satisfaction from the program. For this reason a list of pertinent reprints and references are included at the end of this manual. They should provide assistance to the inexperienced as well as the experienced user. 
INPUT FORMAT FOR TRANSPORT

By the TRANSPORT input DATA SET is meant the totality of data read by the program in a single job. A DATA SET may consist of one or more problems placed sequentially. A problem specifies a calculation or set of calculations to be done on a given beam line.

A problem, in turn, may consist of one or more problem steps. The data in the first step of a problem specify the beam line and the calculations to be made. The data in succeeding steps of the same problem specify only changes to the data given in the first step.

A common example of a problem with several steps is sequential fitting. In the first step one may specify that certain parameters are to be varied to satisfy certain constraints. Once the desired fit has been achieved the program will then proceed to the next step. The data in this step now need specify only which new parameters to vary, or old ones no longer to vary, or which constraints to add or delete. The values of the varied parameters that are passed from one step to the next one are those that are the result of the fitting procedure.

A problem step contains three kinds of DATA cards: the TITLE card, the INDICATOR card, and the ELEMENT cards.

The TITLE card contains a string of characters and blanks enclosed by single quotes. Whatever is between the quotes will be used as a heading in the output of a 'I'RANSPUK'l' run.

The second card of the input is the INDICATOR card. If the data which follow describe a new problem, a zero (0) is punched in any column on the card. If the data which follow describe changes to be made in the previous problem step, a one (1) or minus one $(-1)$ is punched in any column on the card. For further explanation read the Indicator Card section of this manual,

The remaining cards in the deck for a given problem step contain the DATA describing the beam line and the calculations to be done. The DATA concict of a cequenco of elementc whoco order ic the came ac encountored as one proceeds down the beam line. Each element specifies a magnet or 
portion thereof or other piece of equipment, a drift space, the initial beam phase space, a calculation to be done, or a print instruction. Calculation specifications, such as misalignments and constraints, are placed in sequence with the other beam line elements where their effect is to take place. The input format of the cards is "free-field", which is described below. The data for a given problem step are terminated by the word SENTINEL, which need not be punched on a separate card.

Each element, in turn, is given by a sequence of items (mostly numbers), separated by spaces and terminated by a semicolon. The items, in order, are a type code number, a vary field, the physical parameters, and an optional label.

The type code number identifies the element, indicating what sort of entity (such as a magnet, drift space, constraint, etc.) is represented. It is an integer (number) followed by a decimal point. The interpretation of the physical parameters which follow is therefore dependent on the type code number. The type code numbers and their meanings are summarized in Table 1. If the type code number is negative, the element will be ignored in the given problem step. However, storage for that element will be allocated by the program, so that the element may be introduced in a later step of the same problem. Storage space for any element in any problem step must be allocated in the first step of the problem.

The vary field indicates which physical parameters of the element are to be adjusted if there is to be any fitting. It is punched inmediately (no intervening blanks) to the right of the decimal point of the type code number. See the section under type code 10.0 for an explanation of the use of vary codes.

The physical parameters are the quantities which describe the physical element represented. Such parameters may be lengths, magnetic fields, apertures, rotation angles, beam dimensions, or other quantities, depending on the type code number. The meanings for the physical parameters for each type code are described thoroughly in the section for that type code. A summary, indicating the order in which the physical parameters should be punched, is given in Table 1. For any element the first physical parameter is the second entry in Table 1 or the second parameter 
in the section describing a given element. In some cases the parameters of an element do not really refer to physical quantities, but will nevertheless be referred to as such in this manual.

The label, if present, contains one to four characters and is enclosed by single quotes, slashes or equal signs. During the calculation the elements will be printed in sequence and the label for a given element will be printed with that element. Labels are useful in problems with many elements and/or when sequential fitting is used. They must be used to identify any element to be changed in succeeding steps of a given problem.

Provision has been made in the program to allow the user to introduce comments before any type code entry in the data deck. This is accomplished by enclosing the comments made on each card within single parentheses.

Each element must be terminated by a semicolon (;). Optionally a semicolon may be replaced by an asterisk (*) or a dollar sign (\$). Spaces before and after the semicolon are allowed but not required. If the program encounters a semicolon, dollar sign or asterisk before the expected number of parameters has been read in and if the indicator card was a zero (0), the remaining parameters are set to zero. If the indicator card was a one ( 1 ) or minus one $(-1)$, then the numbers indicated on the card are substituted for the numbers remaining from the previous solution; a11 other numbers are unchanged.

The "free-field" input format of the data cards makes it considerably easier to prepare input than the standard fixed-field formats of FORTRAN, Numbers may be punched anywhere on the card and must simply be in the proper order. They must be separated by one or more blanks. Several elements may be included on the same card and a single element may continue from one card to the next. A single number must be all on one card, it may not continue from one card to the next. The program storage is limited to a total of 2000 locations (including type codes and those parameters not punched but implied equal to zero) and 500 elements.

A decimal number (e.g. 2.47) may be represented in any of the following ways: 


\subsection{7 \\ $.00247+3$ \\ $.0247 \mathrm{E}+02$ \\ $247 \mathrm{E}-2$ \\ 247000-5}

The sample problem below contains two problem steps, each beginning with title and indicator cards and terminating with a SENTINEL. The first step causes TRANSPORT to do a first-order calculation with fitting. The second initiates a second-order calculation with the data that is a result of that fitting. Corresponding elements between the two steps are identified by having the same label.

The type ten element which specifies the fitting condition is labelled FIT1. It is active for the first-order calculation, but is turned off for the second-order calculation. The vary codes for elements DRI are set to zero for the second-order problem. The second-order element, SECl, is ineffective during the fitting, but causes the program to compute the second-order matrices in the second calculation. 
An Example of a TRANSPORT Input Deck

'FORTRAN H CHECK ON BETA FI'I'

0

$\begin{array}{lllllllll}1 & .5 & 1 & .5 & 1 & .5 & 1 & 1 & \text {; }\end{array}$

-17 'SEC1' ;

3.32 .745 'DR1';

$20 ; 49.87910 .5 ; 20$

3.32 .745 'DR1' ;

134 ;

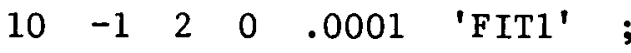

SENTINEL

'SECOND ORDER'

1

$\left.\begin{array}{lll}17 & \text { 'SECl' ; } \\ 3 & \text { 'DRl' ; } \\ -10 & \text { 'FITI' ; }\end{array}\right\}$

SENTTNEL

SENT INEL

Second sentinel signifies end of mon.
Title card

Indicator card

Elements

First problem step

Titie cara

Indicator card

Elements to be changed
Second probzem step

As many problems and problem steps as one wishes may be stacked in one job.

Note that in previous versions of TRANSPORT a decimal point was required with every numerical entry except the indicator card (which musl nut have a decimal point in any version of TRANSPORT). 


\section{The use of labels}

The use of labels is available for identification of individual elements. When inserted for the user's convenience, the association of a label with a given element is optional. If the parameters of an element are to be changed between steps of a given problem, a label is required. The label identifies the element in the earlier step to which the changes specified in the later step are to apply.

The label may be placed anywhere among the parameters of a given element. It should be enclosed in quotes, slashes, or equal signs. Blanks within a label are ignored. The maximum length of a label is four non-blank characters.

As an example, the following all denote the same drift space:

$$
\begin{aligned}
& \text { 'DRF' 3. } 1.5 \text {; } \\
& 3 / \mathrm{DRF} / 15-1 \text { * } \\
& \text { 3. } .15 E 1=\mathrm{DRF}=\$
\end{aligned}
$$
This is to avoid ambiguities with the unit name. Thus the following are not equivalent:

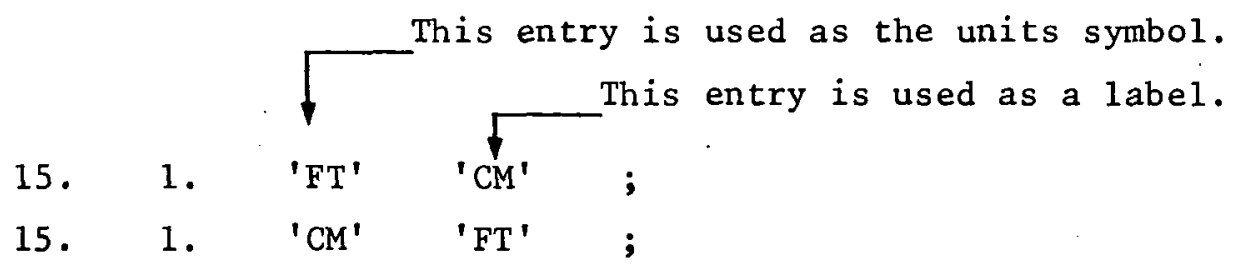

If the parameters describing an element are to differ in succeeding steps of a given problem the element must be included in both steps, having the same label each time. All elements which appear in a problem must be included in the first step (indicator card 0) of that problem. Only those to be changed in later steps need to be labelled. In later steps (indicator card 1) of a problem only those elements to be changed are specified. The elements to be changed are identified by their labels.

If the type code number of an element is negative in a given step of a problem, that element will be ignored when the calculation is performed. However, storage space in the computer will be allocated for the element for possible activation in later steps of the problem. In the later step, only those parameters to be changed need to be specified. 
The storage space allocated for the parameters of a given element is determined only by the type code. The sole exceptions are the continuation codes for type codes 1.0 and 14.0 .

For example, if a fitting constraint is to be ignored in the first step of a problem, but activated in a later step, it should be indicated in both steps. In the first step such an element might appear as

$$
\text { -10. 'FIT' ; }
$$

In the later step one would then insert

$$
\text { 10. 1. 2. } 0.0 \quad .001 \text { 'FII' ; }
$$

causing a waist constraint to be imposed on the beam. Alternatively one can specify the physical parameters in the first step and then, in the later step, merely indicate that the element is now to be activated. The above procedure is therefore equivalent to placing the element:

$$
\text { -10. 1. 2. } 0.0 \quad .001 \quad \text { 'FIT' ; }
$$

in the first problem step, and the element

$$
\text { 10. 'FIT' ; }
$$

in the later step.

Vary codes may also be inserted or removed in passing from one problem step to the next. For instance, one might wish to vary the field of a quadrupole in one step of a problem and then use the fitted value as data in the following step. The first step might then contain the element:

$$
5.01 \quad 5.0 \quad 10.0 \quad 5.0 \quad \text { 'QUAD' }
$$

and the following step would contain the element

$$
\text { 5. 'QUAD' ; }
$$

Since, in the second step, the first item on the card contains no vary code the vary code is deleted. All other parameters, not being respecified, are left unchanged.

Several elements may have the same label. If, as in the above example, one wished to vary the field of several quadrupoles in one step, then pass the final values to the next step, one could give all such 
elements the same label. There might be four quadrupoles, all labelled 'QUAD', being varied simultaneously. If the data for the next step contain the single element

\section{5. 'QUAD' ;}

the vary code on all elements labelled 'QUAD' will be deleted.

\section{Using the program}

All input data must be in the form of 80 byte records. Columns 1-72 are processed by the program, and columns 73-80 are treated as comments. Usually columns 73-80 are used as the line number identification field. The job control language deck setup for operating the program at SLAC is as follows:

// job card information

$/ /$ STEPI EXEC PCM=TRANS

$/ /$ STEPLIB DD DSN=WYL. CG. FXR . L, DISP=SHR

$/ /$ FTO4FOOI DD SYSOUT=B

$/ /$ FTO6FOOI DD SYSOUT $=A$

//FTO5FOOI DD *

transport data deck goes here 
Table 1: Summary of TRANSPORT type codes

\begin{tabular}{|c|c|c|c|c|c|c|c|c|c|}
\hline PHYSICAL ELEMENT & $\begin{array}{l}\text { TrPE } \\
\text { CDEE }\end{array}$ & $\begin{array}{l}\text { 2nd } \\
\text { ENTRY }\end{array}$ & $\begin{array}{l}\text { 3rd } \\
\text { ENTRY }\end{array}$ & $\begin{array}{l}\text { 4th } \\
\text { ENTRY }\end{array}$ & $\begin{array}{l}\text { Sth } \\
\text { ENIRY }\end{array}$ & $\begin{array}{l}\text { 6th } \\
\text { ENIRY }\end{array}$ & $\begin{array}{l}\text { 7th } \\
\text { ENTRY }\end{array}$ & $\begin{array}{l}\text { 8th } \\
\text { ENTRY }\end{array}$ & $\begin{array}{l}\text { 9th } \\
\text { ENIKY }\end{array}$ \\
\hline DEAM & 1.vvovvro & $x(c)$ & $\theta(m r)$ & $y(\mathrm{~cm})$ & $\phi(m r)$ & 2 (an) & $\delta$ (percent) & $\mathrm{P}_{0}$ & \\
\hline $\begin{array}{l}\text { r.m.s. ADDITION TO } \\
\text { BEAM ENELLOPE }\end{array}$ & $1 . v 6 v v v 00$ & $\Delta x(\mathrm{~cm})$ & $\Delta \theta(m x)$ & $\Delta y(c m)$ & $\Delta \phi(m r)$ & $\Delta \ell(\mathrm{cm})$ & $\Delta \delta$ (percent) & $\frac{\Delta P}{(\mathrm{CeV} / \mathrm{c})}$ & 0 \\
\hline POLE FACE ROTATION & $2 . v$ & $\begin{array}{l}\text { ANGLE OF } \\
\text { ROTATION (degrecs) }\end{array}$ & & & & & & & \\
\hline DRIFT & $3 . v$ & LENGTH (metres) & & & & & & & \\
\hline BENDING MAGNET & $4 . \mathrm{vw}$ & LENGTH (metres) & FIELD (kG) & $\begin{array}{l}\text { FIELD GRADIENT } \\
\text { ( } \mathrm{n} \text {-value) }\end{array}$ & & & & & \\
\hline QUADRUPOLE & 5. vv0 & LENGTH (metres) & FIELD (kG) & HALF-APERTURE (Gn) & & & & & \\
\hline TRANSPORM 1 UPDATE & 6.0 & 0.0 & 1.0 & & & & & & \\
\hline TRANSPORM 2 UPDATE & .6 .0 & 0.0 & 2.0 & & & & v & & \\
\hline BEAM CENTROID SHIFT & 7.0 & SHIFT $(x)(\mathrm{cm})$ & SHIFT $(\theta)(m r)$ & SHIFT $(y)(a n)$ & SHIFT $(\phi)(m r)$ & SHIFT $(\ell)(\mathrm{Cm})$ & SHIFT ( $\delta$ percent) & & \\
\hline ALIGNMENT TOLERANGE & 8.vwrvvvo & DISPLACEMENT $(x)(\mathrm{cm})$ & ROTATION $(\theta)(m r)$ & DISPLACEMENT $(y)(\mathrm{cm})$ & ROTATION $(\phi)(m r)$ & DISPLACEMENT $(z)(\mathrm{cm})$ & ROTATION $(\alpha)(\mathrm{mr})$ & $\begin{array}{l}\text { CODE } \\
\text { NUMBR }\end{array}$ & \\
\hline REPEAT CONIRDL & 9.0 & $\begin{array}{l}\text { NUMBER OF } \\
\text { REPEATS }\end{array}$ & & . & & & - & & \\
\hline FITTING CONSTRAINTS & 10.0 & $\pm I$ & $\mathrm{~J}$ & $\begin{array}{l}\text { DESIRED VALUE OF } \\
(\mathrm{I}, \mathrm{J}) \text { MATRIX } \\
\text { ELEMENTS }\end{array}$ & $\begin{array}{l}\text { AOCURACY OF } \\
\text { FIT }\end{array}$ & & & & \\
\hline
\end{tabular}

Noté: +1 is used for fitting a beam (0) matrix element. $\quad-1$ is used for fittịng an Rl matrix element. $-(\mathrm{I}+20)$ is used for fitting an R2 matrix element.

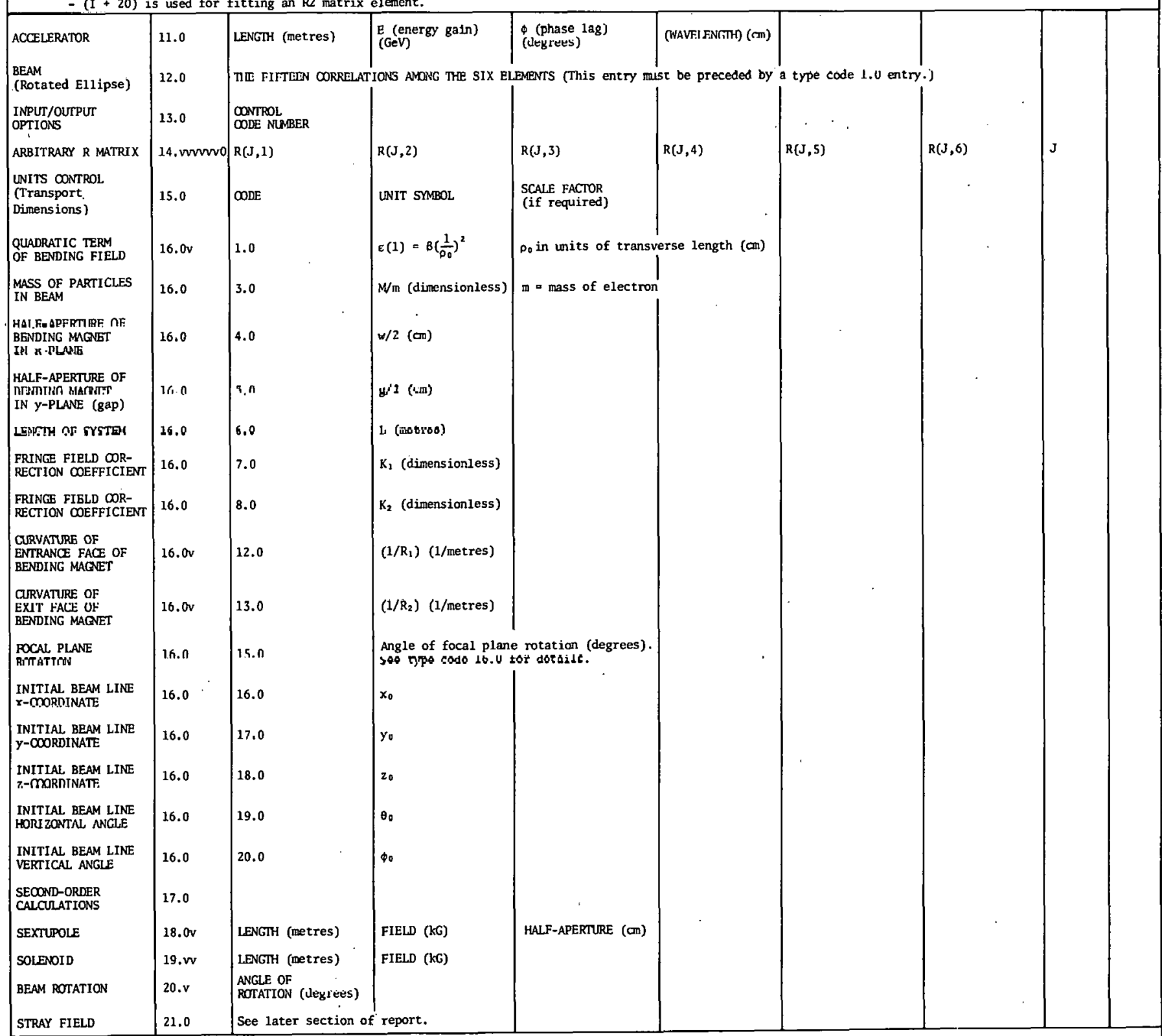

Note: The $v$ 's following the type codes indicate the parameters which may be varied. See section under type code 10.0 for a detailed explanation of 


\section{OUTPUT FORMAT}

\section{General appearance}

Here we give a brief description of the general appearance of the output and its meaning. The user may refer to the sample output shown below $^{*}$ ). It is the printed output resulting from the sample data shown in the section on input format. In a simple example it is not possible to show each of the different type codes. Several of the type codes produce output which is not characteristic of all other type codes. We therefore refer the user to the sections on the various type codes for an explanation of any features peculiar to a given type code.

The output for each step of a given problem is printed separately. The printing for one step is completed before that for the next $s t \in p$ is begun. Therefore we will describe the output for a single problem step. The output shown below is from a problem with two steps.

\section{Initial listing}

For each problem step, the program begins by printing out the user's input records. For the first problem step of a problem, the program also prints. a column of element numbers to the left of the input records. The element numbers provide a unique identification of each element in the system. The element numbers are also printed out whenever the element is printed during calculation. The element number appears between the element name and the type code number on the printout.

*) See page 22 . 


\section{Listing during the calculation}

The program now begins the calculation. If there is no fitting, one listing of the beam line will be made. If there is fitting there will normally be two listings. The first will represent the beam line before any fitting has occurred. The second will be based on the new values of the physical parameters which were altered by the fitting process. If sequential fitting is employed and an indicator card of minus one is used the first run will be omitted. The user should read the section describing the indicator card for further explanation.

In any listing the elements are printed in order with their labels and physical parameters. Elements with negative type code numbers are ignored. Each listed element is preceded by the name of that type of element, enclosed in asterisks. All physical elements are listed in this way. Some of the other elements are not explicitly listed but produce their effect in either the calculated quantities or the listing of the beam line. For descriptions of individual cases, the reader should consult the sections on the type codes.

Calculated quantities appear in the listing as requested in the input data. Important cases will be described in greater detail below. The physical parameters for each element are printed with the appropriate units. For some elements a calculated quantity, not in the input data, will appear, enclosed in parenthesis. Such quantities are explained in the sections under the individual type codes.

\section{Calculated quantities}

The important cases of calculated quantities which appear in the output are the transfer matrices, the beam matrix, the layout courdinates, and the results of the fitting procedure. The transfer and beam matrices and layout coordinares appear as requested in the listing of the beam line. The results of the fitting procedure appear between the two 11 istings. All these quantities are explained in greater detail below.

The transfer matrices appear only where requested. The beam matrix appears automatically after each element, unless suppressed in which case an individual printing may be requested. A request for printing of layout coordinates should be made at the beginning of the beam line. The 
coordinates will then be printed wherever the beam matrix appears. In a11 cases the quantities printed are the values at the interface between two elements. They are evaluated at a point after the element listed above them and before the element listed below. For further explanation of calculated quantities the user should read the section on the mathematical formulation of TRANSPORT, the appendix to the manual, and the section on the appropriate type code. For the transfer matrix the appropriate type code is thirteen; for the beam matrix it is one, and for the coordinate layout it is again thirteen.

Quantities relevant to the fitting appear between the two listings of the beam line. At each iteration of the fitting procedure a line is printed containing the value of the relaxation factor used, the value of chi-squared before the iteration was made, and the corrections made to each of the varied parameters. Once the fitting is complete the final chi-squared and the covariance matrix are printed. For further details the user should read the section on type code 10.0, and the section on fitting in the appendix. 


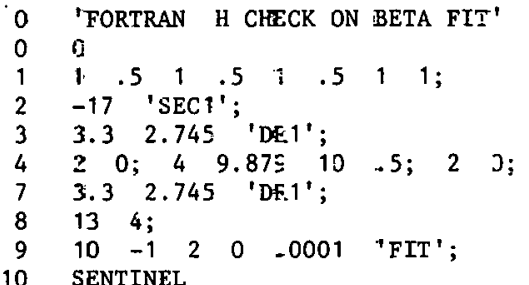

FORTRAN H IHECK ON BETA FIT

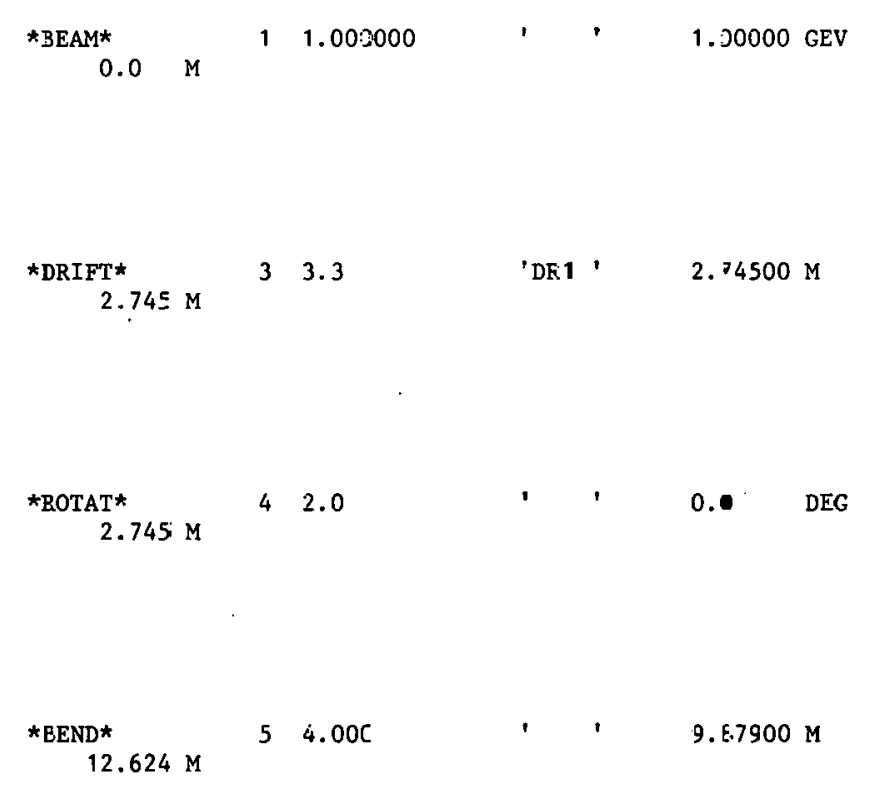

1.
2.
3.
4.
5.
6.
7.
8.
9.
10.

$\begin{array}{ll}0.0 & 0.5000 \mathrm{CM} \\ 0.0 & 1.000 \mathrm{MR} \\ 0.0 & 0.500 \mathrm{CM} \\ 0.0 & 1.000 \mathrm{MR} \\ 0.0 & 0.500 \mathrm{CM} \\ 0.0 & 1.000 \mathrm{PC}\end{array}$

\section{0}

$0.0 \quad 6.0$

c.

$\begin{array}{lll}0.0 & 0.0 & 0.0 \\ 0.0 & C .0 & 0.0\end{array}$

$\begin{array}{llll}0.0 & C .0 & 0.0 & 0.0 \\ 0.0 & C .0 & 0.0 & 0.0\end{array}$

$1.000 \mathrm{PC}$

$\begin{array}{ll}0.0 & 0.573 \mathrm{CM} \\ 0.0 & 1.00 \mathrm{MMR} \\ 0.0 & 0.573 \mathrm{CM}\end{array}$

$0.0 \quad 1.57 \mathrm{CM}$

$\begin{array}{ll}0.0 & 1.003 \mathrm{MR} \\ 0.0 & 0.503 \mathrm{CM}\end{array}$

0.481

$0.0 \quad 0.0$

$\begin{array}{lll}0.0 & 0.0 & \\ 0.0 & 0.0 & 0.481\end{array}$

$\begin{array}{lllll}0.503 \mathrm{CM} & 0.0 & 0.0 & 0.0 & 0.0\end{array}$

$\begin{array}{lllll}0.0 & 0.0 & 0.0 & 0.0 & 0.0\end{array}$

$\begin{array}{ll}0.0 & 0.570 \mathrm{CM} \\ 0.0 & 1.000 \mathrm{MR} \\ 0.0 & 0.570 \mathrm{CM} \\ 0.0 & 1.000 \mathrm{MR} \\ 0.0 & 0.500 \mathrm{CM} \\ 0.0 & 1.000 \mathrm{PC}\end{array}$

$10.00000 \mathrm{KG}$

0.50000

$10.01: \mathrm{CM}$

$12.32 \mathrm{C}$ MR

$0.369 \mathrm{cM}$

$1.360 \mathrm{MR}$

$1.690 \mathrm{CM}$

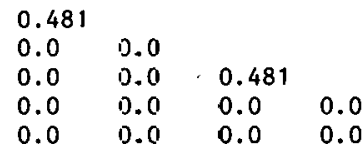

( $169.58 .94 \% \mathrm{DEG}$ )

\subsection{3}

$\begin{array}{lll}0.0 & 0.0 & \\ 0.0 & 0.0 & -0.086\end{array}$

$\begin{array}{llll}-0.992 & -0.9 .2 & 0.0 & 0.0\end{array}$

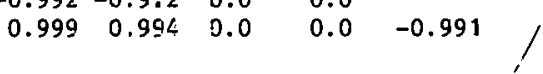

1 


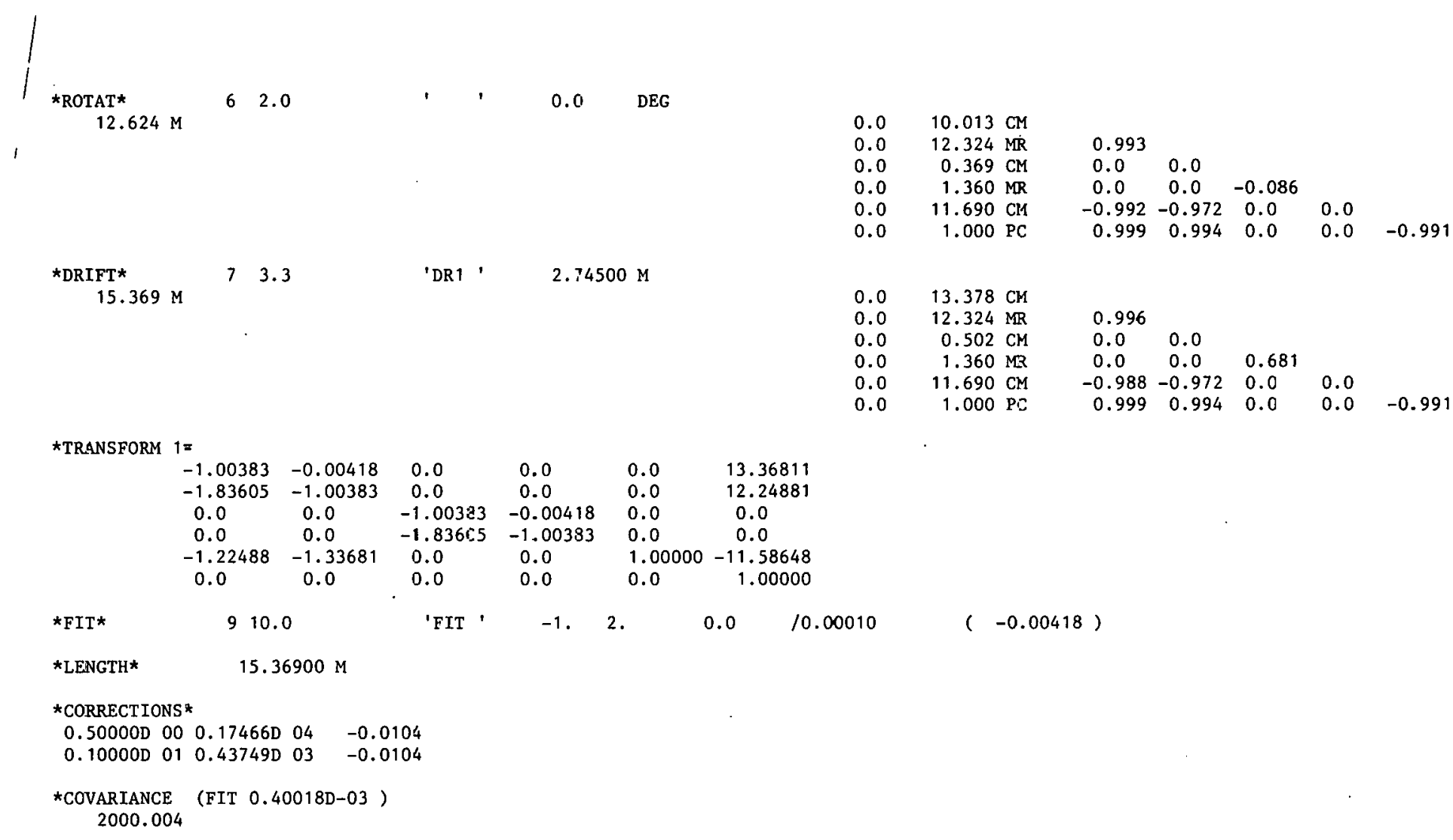




\section{FORERAN H CHECK ON BETA FIT}

$\star_{\text {BEAM* }}$ - 1.000000 $0.0 \quad M$

$2.724 \mathrm{M}$

TR1

$2.72 \div 15 M$

$2.724 \mathrm{M}$

$4 \quad 2.0$

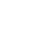

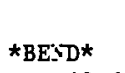

$12.503 \mathrm{M}$

$5 \quad 4.000$

$9.87900 \mathrm{M}$

,

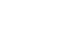

$30.00000 \mathrm{KG}$

$0.0 \quad 0.370 \mathrm{CM}$

0.0 DEG

*ROTAT*

$6 \quad 2.0$

$12.503 \mathrm{M}$

*DRIFT*

$7 \quad 3.3$

$15.327 \mathrm{M}$
73.3
$2.7 .2415 \mathrm{M}$

0.0

0.0
0.0

$0.0 \quad 12.324 \mathrm{MR}$

$1.357 \mathrm{MR}$

$0.0 \quad 1.000 \mathrm{PC}$

$0.500 \mathrm{cM}$

$1.000 \mathrm{MR}$

$0.500 \mathrm{CM}$

$0.500 \mathrm{CM}$

$1.000 \mathrm{PC}$

0.0

$0.0 \quad 0.0$

$\begin{array}{lll}0.0 & 0.0 & 0.0\end{array}$

$\begin{array}{lllll}0.0 & 0.0 & 0.0 & 0.0 & 0.0\end{array}$

$\begin{array}{lll}0.0 & 0.569 \mathrm{CM}\end{array}$

1. $\mathrm{COO} \mathrm{MR}$

$0.569 \mathrm{CM}$

$.600 \mathrm{MR}$

$0.500 \mathrm{CM}$
$1.000 \mathrm{PC}$

$\begin{array}{ll}0.478 & \\ 0.0 & 0.0 \\ 0.0 & 0.0\end{array}$

$0.0 \quad 0.0 \quad 0.478$

$\begin{array}{lll}0.0 & 0.0 & 0.0\end{array}$

$\begin{array}{llll}0.0 & 0.0 & 0.0 & 0.0\end{array}$

$0.569 \mathrm{CM}$

$1.000 \mathrm{MR}$

$0.569 \mathrm{CM}$

$0.500 \mathrm{CM}$

0.478

$0.0 \quad 0.0$

$\begin{array}{lll}0.0 & 0.0 & 0.478\end{array}$

$\begin{array}{llll}0.0 & 0.0 & 0.0 & 0.0\end{array}$

$\begin{array}{lllll}0.0 & 0.0 & 0.0 & 0.0 & 0.0\end{array}$

( 169.68946 DEG )

0.993

$0.0 \quad 0.0$

$\begin{array}{lll}0.0 & 0.0 & -0.086\end{array}$

$\begin{array}{llll}-0.992 & -0.972 & 0.0 & 0.0\end{array}$

$\begin{array}{llllll}0.999 & 0.994 & 0.0 & 0.0 & -0.991\end{array}$

$0.0 \quad 10.013 \mathrm{cM}$

$0.0 \quad 12.324 \mathrm{MR}$

$0.357 \mathrm{MR}$
$11.590 \mathrm{CM}$

$11.590 \mathrm{CM}$

0.993

$\begin{array}{ll}0.0 & 0.0\end{array}$

$\begin{array}{lll}0.0 & 0.0 & -0.086\end{array}$

$\begin{array}{rrrrr}-0.992 & -0.972 & 0.0 & 0.0 & \\ 0.999 & 0.994 & 0.0 & 0.0 & -0.991\end{array}$

$13.352 \mathrm{CM}$
$12.324 \mathrm{MR}$

$0.500 \mathrm{CM}$

$1.357 \mathrm{MR}$

$11.690 \mathrm{CM}$
$1.000 \mathrm{PC}$

0.996

$0.0 \quad 0.0$

$\begin{array}{lll}0.0 & 0.0 & 0.676\end{array}$

$\begin{array}{rrrrr}-0.989 & -0.972 & 0.0 & 0.0 & \\ 0.999 & 0.994 & 0.0 & 0.0 & -0.991\end{array}$ 


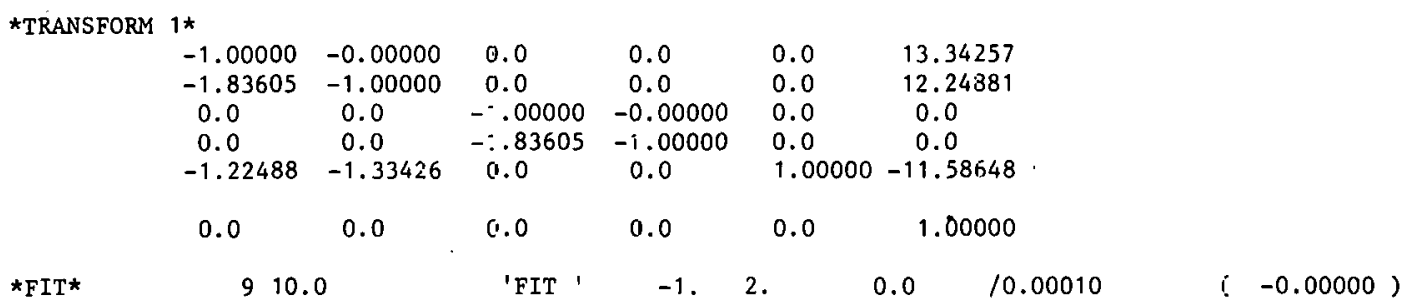

910.0

'FIT '

-1.2 .

0.000010

$(-0.00000)$

*LENGTH*

$15.32731 \mathrm{M}$

$\begin{array}{ll}10 & \text { 'SECOND ORDER' } \\ 10 & 1 \\ 10 & 17 \text { 'SEC1'; } \\ 10 & 3 \text { 'DRT'; } \\ 10 & -10 \text { 'FIT'; } \\ 10 & \text { SENTINEL }\end{array}$

10 SENTINEL

SECOND ORDER

*BEAM* $\quad 1 \quad 1.000000$ $0.0 \quad M$

$\begin{array}{ll}0.0 & 0.500 \mathrm{CM} \\ 0.0 & 1.000 \mathrm{MR} \\ 0.0 & 0.500 \mathrm{CM} \\ 0.0 & 1.000 \mathrm{MR} \\ 0.0 & 0.500 \mathrm{CM} \\ 0.0 & 1.000 \mathrm{PC}\end{array}$

11.
12.
13.
14.
15.
16.

*2ND ORDER* 217

SEC1 ${ }^{\prime} \quad$ GAUSSIAN DISTRIBUTION

*DRIFT*

$3 \quad 3.0$

$2.724 \mathrm{M}$

DR1 '

$2.72415 \mathrm{M}$

$\begin{array}{cc}0.0 & 0.559 \mathrm{CM} \\ 0.0 & 1.000 \mathrm{MR} \\ 0.0 & 0.569 \mathrm{CM} \\ 0.0 & 1.000 \mathrm{MR} \\ -0.000 & 0.500 \mathrm{CM} \\ 0.0 & 1.000 \mathrm{PC}\end{array}$

0.478

$0.0 \quad 0.0$

$\begin{array}{lll}0.0 & 0.0 & 0.478\end{array}$

$0.0 \quad 1.000$ PC

$\begin{array}{lllll}0.0 & 0.0 & 0.0 & 0.0 & \\ 0.0 & 0.0 & 0.0 & 0.0 & 0.0\end{array}$ 


\begin{tabular}{|c|c|c|c|c|c|c|c|c|c|c|c|c|c|c|}
\hline $\begin{array}{l}{ }_{\text {BPOTAT* }}^{*} \\
2.724\end{array}$ & +2 & $\therefore$ & $r$ & 0.0 & DEG & & $\begin{array}{l}0.00 \mathrm{~J} \\
0.0 \\
0.0 \\
0.0 \\
-0.003 \\
0.0\end{array}$ & $\begin{array}{l}0.569 \\
1.000 \\
0.569 \\
1.000 \\
0.500 \\
1.000\end{array}$ & $\begin{array}{l}\mathrm{CM} \\
\mathrm{MR} \\
\mathrm{CM} \\
\mathrm{MR} \\
\mathrm{CM} \\
\mathrm{PC}\end{array}$ & $\begin{array}{c}0.478 \\
0.0 \\
0.0 \\
-0.000 \\
0.0\end{array}$ & $\begin{array}{l}0.0 \\
0.0 \\
0.0 \\
0.0\end{array}$ & $\begin{array}{l}0.478 \\
0.0 \\
0.0\end{array}$ & $\begin{array}{l}0.0 \\
0.0\end{array}$ & 0.0 \\
\hline $\begin{aligned} & * E .3 N D^{\star} \\
& 12.603\end{aligned}$ & 54 & i. & ' & 9.87900 & $M$ & 10.00000 & $\begin{array}{l}\text { IG } \\
0.068 \\
0.037 \\
0.0 \\
0.0 \\
-0.068 \\
0.0\end{array}$ & $\begin{array}{r}0.5000 \\
10.013 \\
12.324 \\
0.370 \\
1.358 \\
11.690 \\
1.000\end{array}$ & $\begin{array}{l}00 \\
C M \\
M R \\
C M \\
M R \\
C M \\
P C\end{array}$ & $\begin{array}{c}C \quad 169 . \\
0.793 \\
0.0 \\
0.0 \\
-0.992 \\
0.999\end{array}$ & $\begin{array}{l}68946 \mathrm{D} \\
\\
0.0 \\
0.0 \\
-0.972 \\
0.994\end{array}$ & $\begin{array}{l}\text { DEG ) } \\
\begin{array}{l}-0.086 \\
0.0 \\
0.0\end{array}\end{array}$ & $\begin{array}{l}0.0 \\
0.0\end{array}$ & -0.991 \\
\hline $\begin{array}{l}\text { *DTAT }{ }^{*} \\
12.603\end{array}$ & 62 & 2. & ' & $0 . c$ & DEG & & $\begin{array}{l}0.068 \\
-0.0 \Xi 7 \\
0.0 \\
0.0 \\
-0.062 \\
0.0\end{array}$ & $\begin{array}{r}10.013 \\
12.324 \\
0.370 \\
1.358 \\
11.690 \\
1.000\end{array}$ & $\begin{array}{l}\mathrm{CM} \\
\mathrm{MR} \\
\mathrm{CM} \\
\mathrm{MR} \\
\mathrm{CM} \\
\mathrm{PC}\end{array}$ & $\begin{array}{c}0.993 \\
0.0 \\
0.0 \\
-0.992 \\
0.999\end{array}$ & $\begin{array}{c}0.0 \\
0.0 \\
-0.972 \\
0.994\end{array}$ & $\begin{array}{l}-0.086 \\
0.0 \\
0.0\end{array}$ & $\begin{array}{l}0.6 \\
0.0\end{array}$ & -0.991 \\
\hline $\begin{array}{l}\text { *IRIFT* } \\
15.327\end{array}$ & 73 & 3.0 & 'DR1 & 2. .2415 & $M$ & - & $\begin{array}{l}0 \\
0.0: 8 \\
0.0 \vdots 7 \\
0.0 \\
0.0 \\
-0.0 \div 3 \\
0.0\end{array}$ & $\begin{array}{r}13.352 \\
12.324 \\
0.500 \\
1.358 \\
11.690 \\
1.000\end{array}$ & $\begin{array}{l}\mathrm{CM} \\
\mathrm{MR} \\
\mathrm{CM} \\
\mathrm{MR} \\
\mathrm{CM} \\
\mathrm{PC}\end{array}$ & $\begin{array}{l}0.996 \\
0.6 \\
0.0 \\
-0.988 \\
0.599\end{array}$ & $\begin{array}{c}0.0 \\
0.0 \\
-0.972 \\
0.994\end{array}$ & $\begin{array}{l}0.676 \\
0.0 \\
0.0\end{array}$ & $\begin{array}{l}0.0 \\
0.0\end{array}$ & -0.991 \\
\hline * TRANSFORM & $\begin{array}{l}1 * \\
-1.000(0 \\
-1.336(.5 \\
0.1) \\
0.1 \\
-1.22488 \\
0.3\end{array}$ & $\begin{array}{l}-0.0000 \mathrm{r} \\
-1.0000 \mathrm{C} \\
0.0 \\
0.0 \\
-1.33426 \\
0.0\end{array}$ & $\begin{array}{l}0.0 \\
0.0 \\
-1.00000 \\
-1.83605 \\
c .0 \\
6.0\end{array}$ & $\begin{array}{l}0.0 \\
0.0 \\
-0.000100 \\
-1.000100 \\
0.0 \\
0.0\end{array}$ & $\begin{array}{l}0.0 \\
0.0 \\
0.0 \\
0.0 \\
1.00000 \\
0.0\end{array}$ & $\begin{array}{r}13.34257 \\
12.24881 \\
0.0 \\
0.0 \\
-11.58648 \\
1.00000\end{array}$ & & & & & & & & \\
\hline
\end{tabular}




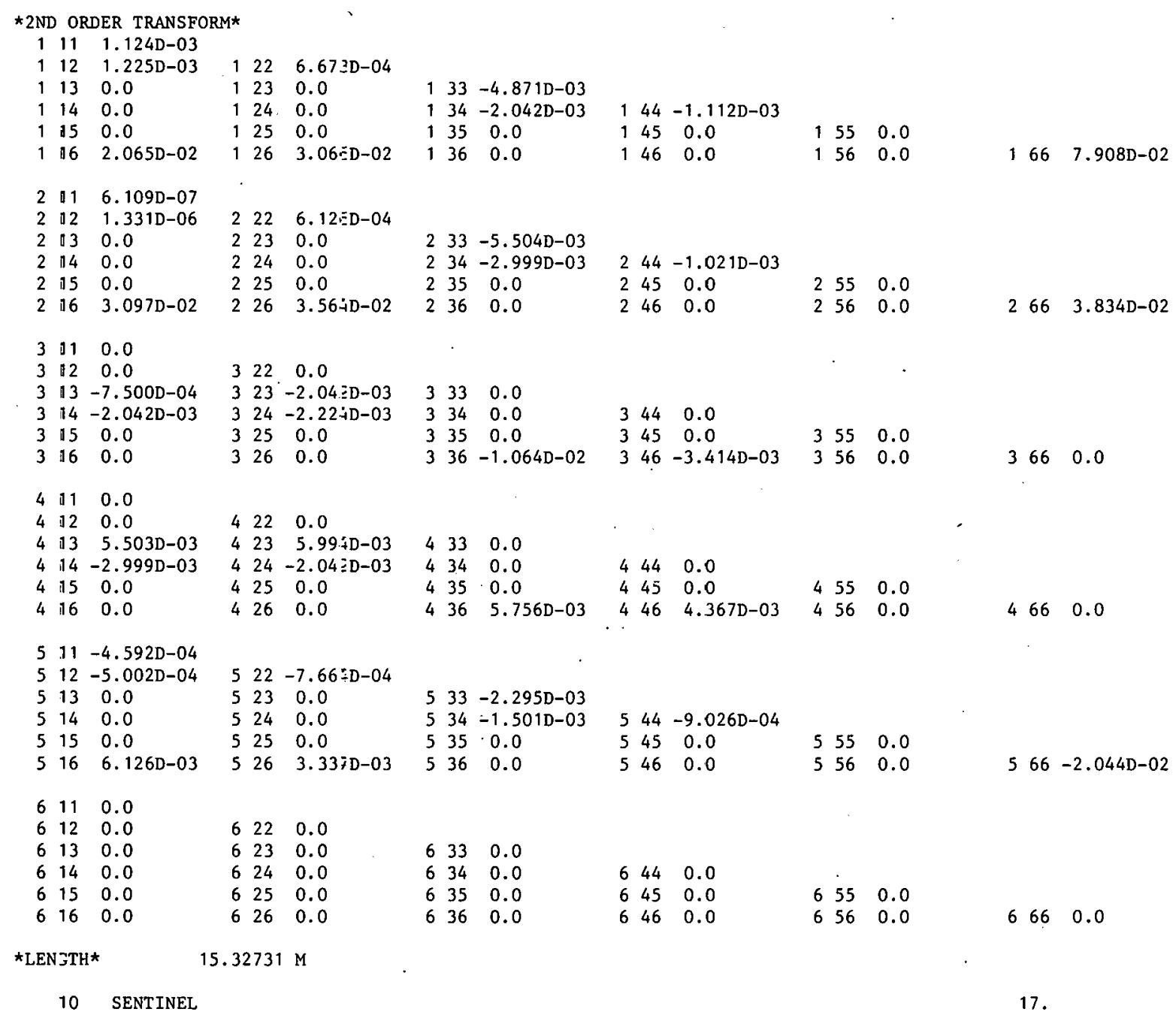




\section{TITLE CARD}

The title card is the first card in every problem step of a TRANSPORT data set. The title card is always required and must be followed by a $\underline{0, a+1}$ or -1 card (see next section) to indicate whether the data to follow is new ( 0 card) or a continuation of a previous data set ( \pm 1 card).

The title must be enclosed within either quotation marks ('), slashes $(/)$, or equal signs $\Leftrightarrow$ on a single card. The string may begin and end in any column (free field format), for example

'SLAC $20 \mathrm{GEV} / \mathrm{C}$ SPECTROMETER'

or

/SLAC $20 \mathrm{GEV}$ SPECTROMETER/

Note that whichever character is used to enclose the title must not be used again within the title itself.

Exarple of a DATA SET for a single problem step

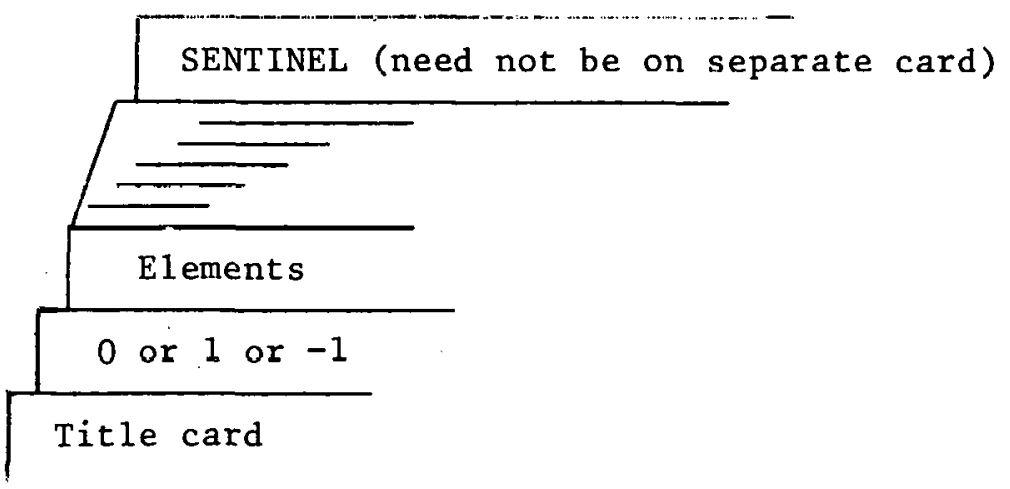




\section{INDICATOR CARD ( 0 or \pm 1$)$}

The second card of the input for each step of a problem is the indicator card. If the data which follow describe a new problem, a zero (0) is punched in any colum on the card. If the data which follow describe changes to be made in the previous step of a given problem, a one (1) or minus one $(-1)$ is punched in any column on the card.

If a given problem step involves fitting, the program will normally list the beam line twice, printing each time the sequence of elements along with transfer or beam matrices where specified. The first listing uses the parameters of each element before any fitting has taken place. The second shows the results of the fitting. If a problem involving fitting has several steps, the second run of a given step often differs little from the first run of the following step.

If the second or subsequent step of a problem involves fitting and one wishes to print both runs through the beam line, a one (1) is punched on the indicator card. If the first listing is to be suppressed a minus one $(-1)$ is punched. If no fitting is involved, the program will ignore the minus sign and will do one single run through the system.

The sample problem input shown on page 14 causes TRANSPORT to do a first-order calculation with fitting ( 0 indicator card) and then to do a second-order calculation ( 1 indicator card) with the data that is the result of the fitting. 


\section{COMMENT CARDS}

Comment cards may be introduced anywhere in the deck where an element would be allowed by enclosing the comments made on each card within single parentheses. No parentheses are allowed within the parentheses of any comment card. The comments are not stored, but appear only in the initial listing of the given problem step.

Example of the use of comment cords in a data set

'Title Card'

0

(THIS IS A TEST PROBLEM TO ILLUSTRATE THE)

(USE OF COMMENT CARDS)

elements

(COMMENTS MAY ALSO BE MADE BETWEEN)

(TYPE CODE ENTRIES)

elements

SENTINEL 


\section{LISTING OF AVAILABLE TRANSPORT TYPE CODE ENTRIES}

INPUT BEAM: Type code 1.0

The phase space and the average momentum of the input beam for a TRANSPORT calculation are specified by this element. The input is given in terms of the semi-axes of a six-dimensional erect ${ }^{*}$ ) beam ellipsoid representing the phase space variables $x, \theta, y, \phi, \ell$, and $\delta$. Each of these six parameters is entered as a positive quantity, but should be thought of as $\pm x, \pm \theta$, etc; hence, the total beam width is $2 x$, the total horizontal beam divergence is $2 \theta$ and so forth.

Usually the BEAM card is the third card in the deck. If other than standard TRANSPORT units are to be used, the units specification cards (type code 15.0) should precede the BEAM card. Standard TRANSPORT units for $\mathrm{x}, \theta, \mathrm{y}, \phi, \ell$, and $\delta$ are $\mathrm{cm}, \mathrm{mr}, \mathrm{cm}, \mathrm{mr}, \mathrm{cm}$ and percent. The standard unit for the momentum $\mathrm{p}(0)$ is $\mathrm{GeV} / \mathrm{c}$. Also if a beam line coordinate layout is desired, the card specifying that a layout is to be made (a 13.0 12.0 element), and any initial coordinates (see type code 16.0) all precede the BEAM card.

There are eight entries (all positive) to be made on the BEAM card.

1 - The type code 1.0 (specifies a BEAM entry follows).

2 - One-half the horizontal beam extent ( $x$ ) (cm in standard units).

3 - One-half the horizontal beam divergence $(\theta)$ ( $\mathrm{mr}$ ).

4 - One-half the vertical beam extent $(y)(\mathrm{cm})$.

5 - One-half the vertical heam divergence $(\phi)$ (mr).

6 - One-half the longitudinal beam extent (l) (cm).

7 - One-half the momentum spread $(\delta)$ (in units of percent $\Delta p / p$ ).

8 - The momentum of the central trajectory $[\mathrm{p}(0)](\mathrm{GeV} / \mathrm{c})$.

A11 eight entries must be made even if they are zero (0). As for all other type codes, the last entry must be followed by a semicolon, dollar sign, or asterisk. Thus a typical BEAM entry might be
1. $\quad 0.52$.
1.3
2.50
1.5 Label if $\sqrt{,}$ desired.

*) For a rotated (non-erect) phase ellipsoid input, see type code 12.0 . 
meaning, $x= \pm 0.5 \mathrm{~cm}, \theta= \pm 2.0 \mathrm{mr}, y= \pm 1.3 \mathrm{~cm}, \phi= \pm 2.5 \mathrm{mr}, \ell= \pm 0.0 \mathrm{~cm}$, $\delta= \pm 1.5$ percent $\Delta \mathrm{p} / \mathrm{p}$, and the central momentum $\mathrm{p}(0)=10.0 \mathrm{GeV} / \mathrm{c}$.

The units of the tabulated matrix elements in either the firstorder $\mathrm{R}$ or sigma matrix or second order $\mathrm{T}$ matrix of a TRANSPORT print-out will correspond to the units chosen for the BEAM card. For the above example, the $R(12)=(x / \theta)$ matrix element will have the dimensions of $\mathrm{cm} / \mathrm{mr}$; and the $\mathrm{T}(236)=(\theta / \mathrm{y} \delta)$ matrix element will have the dimensions $\mathrm{mr} /(\mathrm{cm} \cdot$ percent $)$ and so forth.

The longitudinal extent $\ell$ is useful for pulsed beams. It indicates the spread in length of particles in a pulse. It does not interact with any other component and may be set to zero if the pulac langth is not important.

The phase ellipse (sigma matrix) beam parameters are normally printed as output after each physical element unless suppressed by a (13. 2. ;) element. The projection of the semi-axes of the ellipsoid upon each. of its six coordinates axes is printed in a vertical array and the correlations among these components indicating the phase ellipse orientations are printed in a triangular array (see the following pages). 
The phase ellipse beam matrix

The beam matrix carried in the computer has the following construction:

\begin{tabular}{c|cccccc} 
& \multicolumn{1}{|c}{$\mathbf{x}$} & $\theta$ & $\mathbf{y}$ & $\phi$ & $\ell$ & $\delta$ \\
\hline $\mathbf{x}$ & $\sigma(11)$ & & & & & \\
$\theta$ & $\sigma(21)$ & $\sigma(22)$ & & & & \\
$\mathbf{y}$ & $\sigma(31)$ & $\sigma(32)$ & $\sigma(33)$ & & & \\
$\theta$ & $\sigma(41)$ & $\sigma(42)$ & $\sigma(43)$ & $\sigma(44)$ & & \\
$\ell$ & $\sigma(51)$ & $\sigma(52)$ & $\sigma(53)$ & $\sigma(54)$ & $\sigma(55)$ & \\
$\delta$ & $\sigma(61)$ & $\sigma(62)$ & $\sigma(63)$ & $\sigma(64)$ & $\sigma(65)$ & $\sigma(66)$
\end{tabular}

The matrix is symmetric so that only a triangle of elements is needed.

In the printed output this matrix has a somewhat different format for ease of interpretation:

\begin{tabular}{|c|c|c|c|c|c|c|c|}
\hline & & & $x$ & $\theta$ & $\mathrm{y}$ & $\phi$ & $\ell$ \\
\hline$x$ & $\sqrt{\sigma}(11)$ & $\mathrm{CM}$ & & & & & \\
\hline$\theta$ & $\sqrt{\sigma}(22)$ & MR & $r(21)$ & & & & \\
\hline$y$ & $\sqrt{\sigma}(33)$ & $\mathrm{CM}$ & $r(31)$ & $r(32)$ & & & \\
\hline$\phi$ & $\sqrt{\sigma}(44)$ & MR & $r(41)$ & $r(42)$ & $r(43)$ & & \\
\hline$\ell$ & $\sqrt{0}(55)$ & LM & $r(b 1)$ & $r(52)$ & $r(53)$ & $r(54)$ & \\
\hline$\delta$ & $\sqrt{\sigma}(66)$ & $\mathrm{PC}$ & $r(61)$ & $r(62)$ & $r(63)$ & $r(64)$ & $r(65)$ \\
\hline
\end{tabular}

where: $\quad r(i j)=\frac{\sigma(i j)}{[\sigma(i i) \sigma(j j)]^{\frac{1}{2}}}$.

As a result of the fact that the $\sigma$ matrix is positive definite, the $r(i j)$ satisfy. the relation 
The full significance of the $\sigma(i j)$ and the $r(i j)$ are discussed in detail in the Appendix ("Description of Beam Matrix"). The units are always printed with the matrix.

In brief, the meaning of the $\sqrt{\sigma}(\mathrm{ii})$ is as follows:

$\sqrt{\sigma}(11)=x_{\max }=$ the maximum (half)-width of the beam envelope in the $x$ (bend)-plane at the point of the print-out.

$\sqrt{\sigma}(22)=\theta_{\max }=$ the maximum (half)-angular divergence of the beam envelope in the $x$ (bend) plane.

$\sqrt{\pi}(3.3)=\mathrm{y}_{\max }=$ the maximum (half) -height of the beam envelope.

$\sqrt{\sigma}(44)=\phi_{\max }=$ the maximum (half)-angular divergence of the beam envelope in the $y$ (non-bend)-plane.

$\sqrt{\sigma}(55)=\ell_{\max }=$ one-half the longitudinal extent of the bunch of particles.

$\sqrt{\sigma}(66)=\delta \quad=$ the half-width $(1 / 2 \Delta \mathrm{p} / \mathrm{p})$ of the momentum interval being transmitted by the system.

The units appearing next to the $\sqrt{\sigma}(i i)$ in the TRANSPORT print-out are the units chosen for coordinates $\mathrm{x}, \theta, \mathrm{y}, \phi, \ell$ and $\delta=\Delta \mathrm{p} / \mathrm{p}$, respectively.

To the immediate $1 \mathrm{pft}$ of the listing of the beam cnvelope size in a TRANSPORT print-out, there appears a column of numbers whose values will normally be zero. 'l'hese numbers are lle cuvidinates of the controid nf the beam phase ellipse (with respect to the initially assumed central trajectory of the system). They may become non-yero under one of three circumstances:

1) when the misalignment (type code 8.0) is used,

2) when a hcam controid shift (type rode 7.0) is used, or

3) when a second-order calculation (type code 17.0) is used. 
To aid in the interpretation of the phase ellipse parameters listed above, an example of an $(x, \theta)$ plane ellipse is illustrated below. For further details the reader should refer to the Appendix of this report.

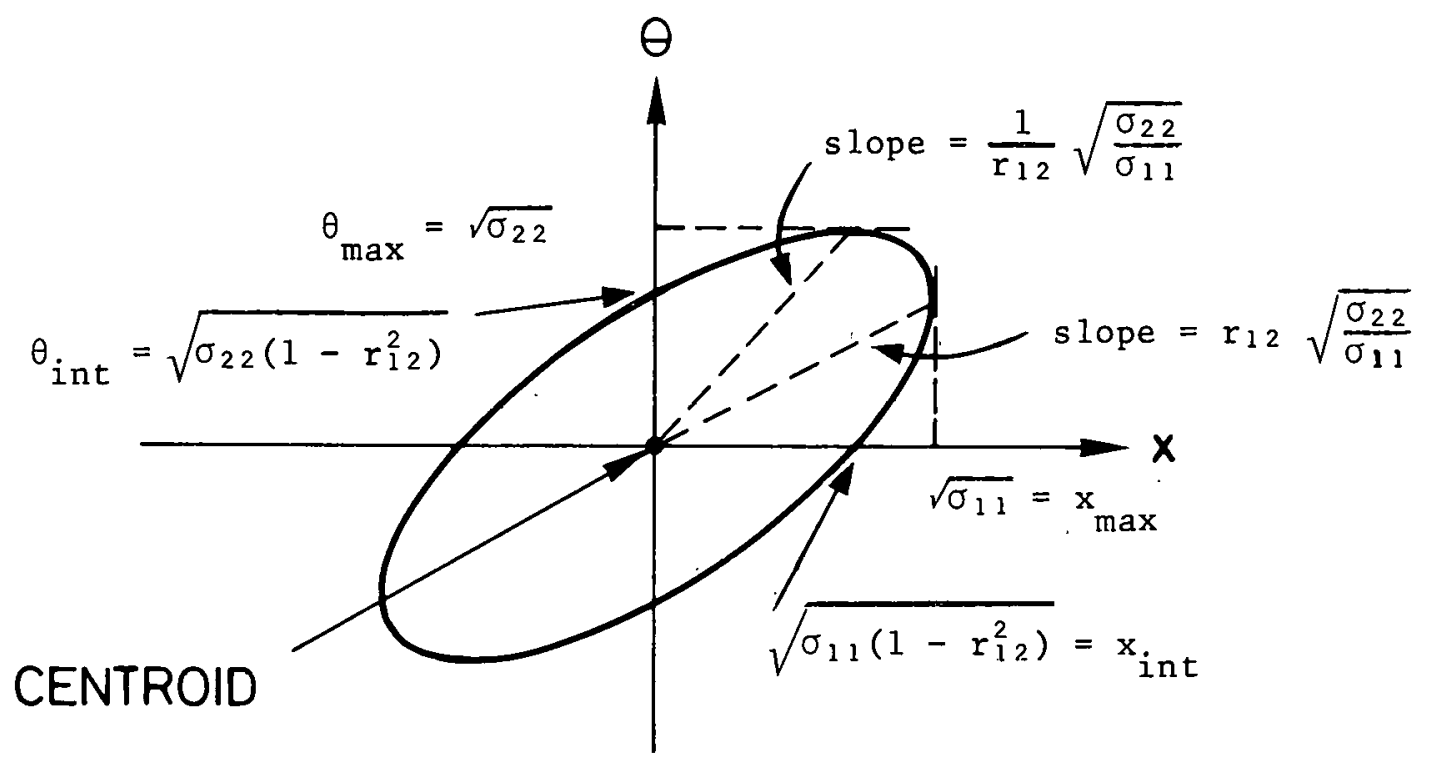

A TWO-DIMENSIONAL BEAM PHASE ELLIPSE

The area of the ellipse is given by:

$$
A=\pi(\operatorname{det} \sigma)^{\frac{1}{2}}=\pi x_{\text {illdx }} \theta_{\text {int }}=\pi x_{\text {in } \tau} \theta_{\max }
$$


r.m.s. addition to the BEAM

To allow for physical phenomena such as multiple scattering, provision has been made in the program to permit an r.m.s. addition to the beam envelope. There are nine entries to be included:

1 - Type code 1.0 (specifying a BEAM entry follows).

2 - The r.m.s. addition to the horizontal beam extent $(\Delta \mathrm{x})(\mathrm{cm})$.

3 - The r.m.s. addition to the horizontal beam divergence $(\Delta \theta)(\mathrm{mr})$.

4 - The r.m.s. addition to the vertical beam extent $(\Delta y)(\mathrm{cm})$.

5 - The r.m.s. addition to the vertical beam divergence $(\Delta \phi)(\mathrm{mr})$.

6 - The r.m.s. longitudinal beam extent $(\Delta l)(\mathrm{cm})$.

7 - The r.m.s. momentum spread $(\Delta \delta$ ) (in percent $\Delta p / p$ ).

8 - The momentum change in the central trajectory $[\Delta \mathrm{p}(0)]$ in $(\mathrm{GeV} / \mathrm{c})$.

9 - The code digit 0 . indicating an r.m.s. addition to the BEAM is being made.

The units for the r.m.s. addition are the same as those selected for a regular BEAM type code 1.0 entry. Thus a typical r.m.s. addition to the BEAM would appear as follows:

1. $\begin{array}{lllllllll} & 1 & .2 & .15 & .3 & 0 . & .13 & -0.1 & 0 .\end{array}$

where the last entry ( 0.$)$ preceding the semicolon signifies an r.m.s. addition to the BEAM is being made and the next to the last entry indirates a central momentum change of $-0.1 \mathrm{GeV} / \mathrm{c}$. 
FRINGING FIELDS and POLE-FACE ROTATIONS for bending magnets:

Type code 2.0

To provide for fringing fields and/or pole-face rotations on bending magnets, the type code 2.0 element is used.

There are two parameters:

1 - Type code 2.0 .

2 - Angle of pole-face rotation (degrees).

The type code 2.0 element must either immediately precede a bending magnet (type code 4.0) element (in which case it indicates an entrance fringing field and pole-face rotation) or immediately follow a type code 4.0 element (exit fringing field and pole-face rotation) with no other data entries between ${ }^{*}$. A positive sign of the angle on either entrance or exit pole-faces corresponds to a non-bend plane focusing action and bend plane defocusing action.

For example, a symmetrically oriented rectangular bending magnet whose total bend is 10 degrees would be represented by the three entries 2. 5. ; 4. -- ; 2. 5. ;

The angle of rotation may be varied. For example, the element 2.15 5. ; would allow the angle to vary from an initial guess of 5 degrees to a final value which would, say, satisfy a vertical focus constraint imposed upon the system. See the type code 10.0 section for a complete discussion of vary'codes.

Even if the pole-face rotation angle is zero, 2. 0. ; entries must be included in the data set before and after a type code 4.0 entry if fringing-field effects are to be calculated.

A single type code 2.0 entry that follows one bending magnet and precedes another will be associated with the latter.

*) It is extremely important that no data entries be made between a type code 2.0 and a type code 4.0 entry. If this occurs, it may result in an incorrect matrix multiplication in the program and hence an incorrect physical answer. If this rule is violated, an error message will be printed. 
Should it be desired to misalign such a magnet, an update element must be inserted immediately before the first type 2.0 code entry and the convention appropriate to misalignment of a set of elements applied, since, indeed, three separate transformations are involved. See section under type code 8.0 for a discussion of misalignment calculations and the section under type code 6.0 for a discussion of updates.

The type code signifying a rotated pole-face is 2.0 . The input format is:

$$
\text { 2. B. 'RO' }
$$

The units for $\beta$ are degrees. 


\section{Pole-face rotation matrix}

The first-order $R$ matrix for a pole-face rotation used in a TRANSPORT calculation is as follows:

$$
R=\left(\begin{array}{cccccc}
1 & 0 & 0 & 0 & 0 & 0 \\
\frac{\tan \beta}{\rho_{0}} & 1 & 0 & 0 & 0 & 0 \\
0 & 0 & 1 & 0 & 0 & 0 \\
0 & 0-\frac{\tan (\beta-\psi)}{\rho_{0}} & 1 & 0 & 0 \\
0 & 0 & 0 & 0 & 1 & 0 \\
0 & 0 & 0 & 0 & 0 & 1
\end{array}\right)
$$

Definitions: $\quad B=$ angle of rotation of pole face (see figure on following page for sign convention of $B$ )

$$
\begin{aligned}
\rho_{0}= & \text { bending radius of central trajectory } \\
g= & \text { total gap of magnet } \\
\psi= & \text { correction term resulting from spatial extent of } \\
& \text { fringing fields } \left.{ }^{*}\right) .
\end{aligned}
$$

where

$$
\psi=K_{1}\left(\frac{g}{\rho_{0}}\right)\left(\frac{1+\sin ^{2} \beta}{\cos \beta}\right)\left[1-K_{1} K_{2}\left(\frac{g}{\rho_{0}}\right) \tan \beta\right]^{*}
$$

\footnotetext{
${ }^{\star}$ See type code 16.0 for input formats for $g, K_{1}$, and $k_{2}$ TRANSPORT entries.

${ }^{* *}$ See SLAC-75) (page 74) for a discussion of $\psi$.
} 


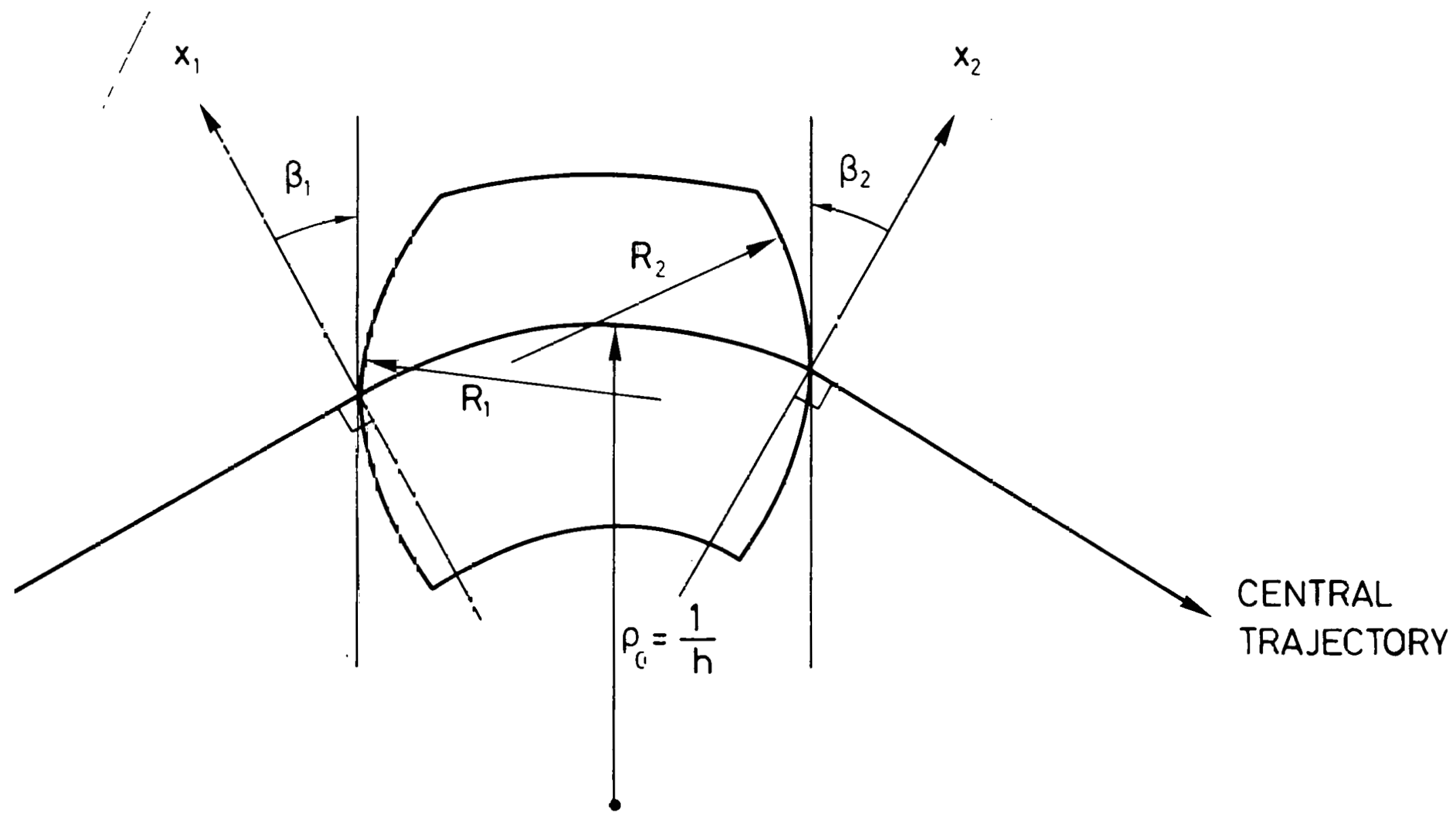

FIELL BOLNDARIES FOR BENDING MAGNETS

The TRANSPORT $\leq i g n$ conventions for $x, \beta, R$ and $h$ are all positive as shown in the figure. The positive $y$ direction is out of the paper. Positive $\beta^{\prime}$ s imply transverse focusing. Positive $R^{\prime} s$ (convex curratures) represent negative sextupole componenis oi strength $S=(-h / 2 R) s \in c^{3} \beta . \quad(S \in \equiv S L A C-75$, page 71.) 
DRIFT: Type code 3.0

A drift space is a field-free region through which the beam passes. There are two parameters:

1 - Type code 3.0 (specifying a drift length).

2 - (Effective) drift length (metres). The length of a drift space may be varied in either first- or second-order fitting.

Typical input format for a DRIFT:

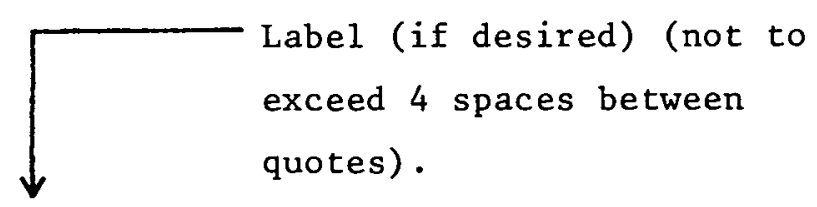

\section{6. 'D1' ;}

\section{DRIFT space matrix}

The first-order $R$ matrix for a drift space is as follows:

$\left[\begin{array}{llllll}1 & \text { L } & 0 & 0 & 0 & 0 \\ 0 & 1 & 0 & 0 & 0 & 0 \\ 0 & 0 & 1 & \mathrm{~L} & 0 & 0 \\ 0 & 0 & 0 & 1 & 0 & 0 \\ 0 & 0 & 0 & 0 & 1 & 0 \\ 0 & 0 & 0 & 0 & 0 & 1\end{array}\right]$

where

$$
L=\text { the length of the drift space. }
$$

The dimensions of $\mathrm{L}$. are those chosen for longitudinal length via a

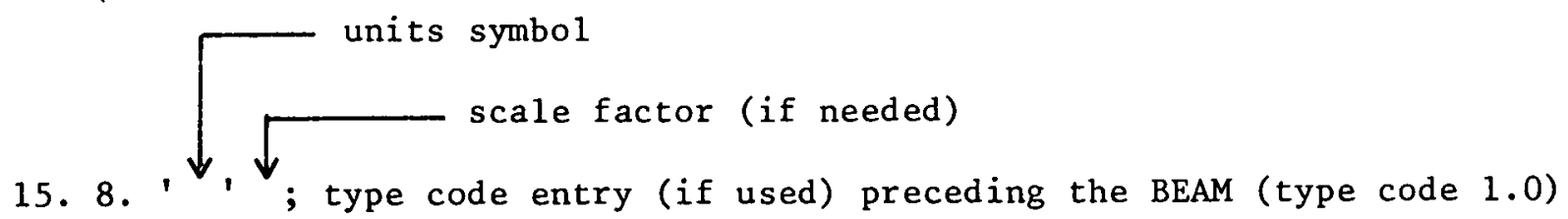
card. If no 15. 8. entry is made, the units of L. will automatically be in metrcs (etandard TRANSPORT units). 
WEDGE BENDING MAGNET: Type code 4.0

A wedge bending magnet implies that the central trajectory of the beam enters and exits perpendicularly to the pole-face boundaries (to include fringing-field effects and non-perpendicular entrance or exit boundaries -- see type codes 2.0 and 16.0 ).

There are four first-order parameters to be specified for the wedge magnet via type code 4.0 :

1 - Type code 4.0 (specifying a wedge bending magnet).

2 - The (effective) length $L$ of the central trajectory in metres.

3 - The central field strength $B(0)$ in $k G$,

$$
B(0)=33.356\left(p / \rho_{0}\right) \text {, }
$$

where $p$ is the momentum in $\mathrm{GeV} / \mathrm{s}$ and $\rho_{0}$ is the bending radiuc of the central trajectory in metres.

4 - The field gradient (n-value, dimensionless); where $n$ is defined by the equation

where

$$
B_{y}(x, 0, t)=B_{y}(0,0, t)(1-n h x+\ldots),
$$

$$
h=1 / \rho_{0} . \text { See SLAC-75 (page 31) }{ }^{4} \text { ). }
$$

The quantities $L, B(0)$, and $n$ may be varied for first-order fitting (see type code 10.0 for a discussion of vary codes).

The bend angle in degrees is printed in the output.

A typical first-order TRANSPORT input for a wedge magnet is

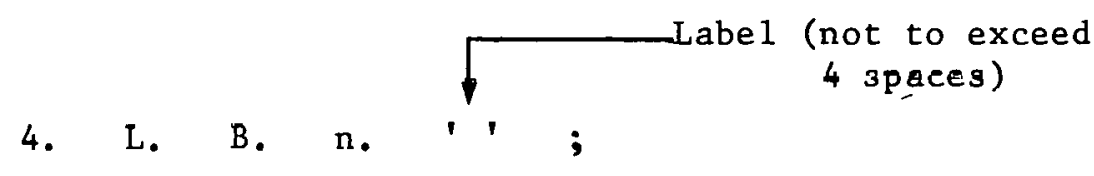

If fringing fleld effects are to be included, a type code 2.0 entry must immediately precede and follow the pertinent type code 4.0 entry (even if there are no pole-face rotations). Thus a typical TRANSPORT input for a bending magnet including fringing fields might be:

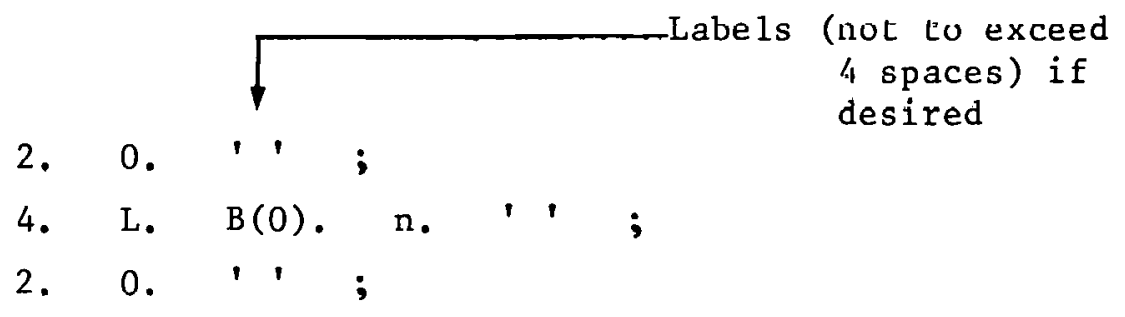


For non-zero pole-face rotations a typical data input might be

2. $10 . \quad$ 4. L. $B(0)$. n. ; 2. 20. ;

Note that the use of labels is optional and that all data entries may be made on one line if desired.

The sign conventions for bending magnet entries are illustrated in the following figure. For TRANSPORT a positive bend is to the right looking in the direction of particle travel. To represent a bend in another sense, the coordinate rotation matrix (type code 20.0 ) should be used as follows:

A bend up is represented by rotating the ( $x, y)$ coordinates by -90.0 degrees about the $(z)$ beam axis as follows:

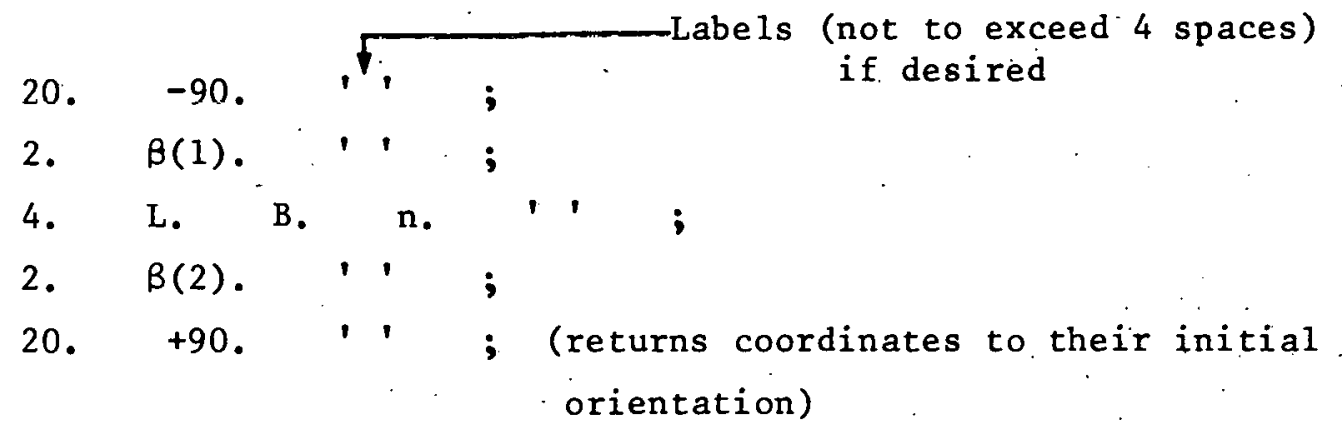

\section{A bend down is accomplished via:}

20. +90 .

2 .

4.

2.

20.

-90 .

;

A bend to the left (looking in the direction of beam travel) is accomplished by rotating the $x, y$ coordinates by 180 degrees, e.g.

20. 180. ' ' ;

2.

4.

2.

20. -180 ' ' ; 


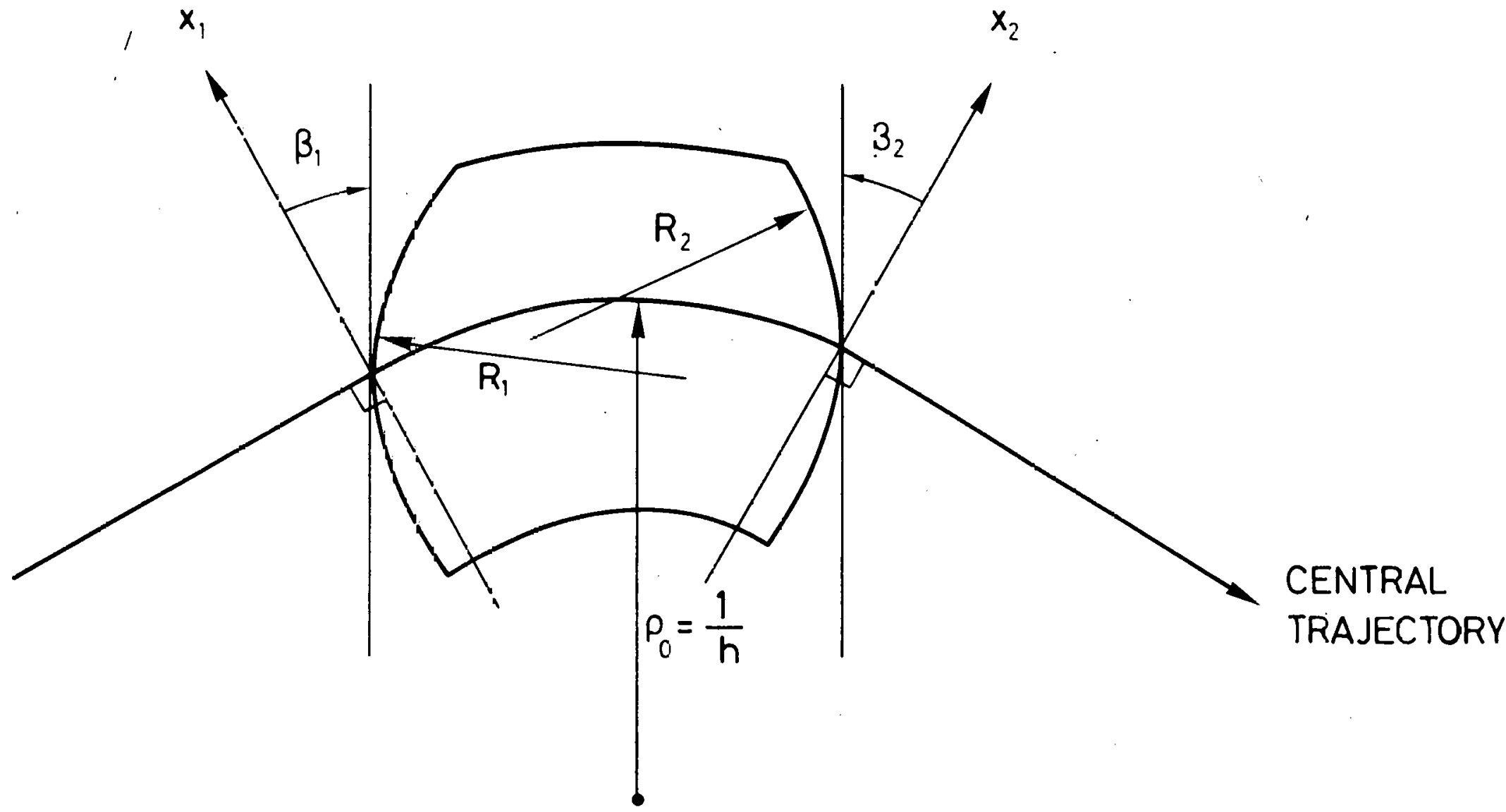

FIELJ BOUADARIES FOR BENDING MAGNETS

The TFANSPORT sign conventions for $\underline{x}, \beta, R$ and $h$ are all positive as shown in the figure. The positive $y$ lirection is out of the paper. Positive $\beta^{\prime}$ s imply tramsverse focusing.

Positive R's (convex curvatires) represent negative sextupole components of strength $S=(-h / 2 R) \operatorname{se}^{3} B$. (See illAC-75, page 71.) 


\section{First-order weäge bending magnet matrix}

\begin{tabular}{|c|c|c|c|c|c|}
\hline $\cos k_{x} L$ & $\frac{1}{k_{x}} \sin k_{x} L$ & 0 & 0 & 0 & $\frac{h}{k_{x}^{2}}\left(1-\cos k_{x} L\right)$ \\
\hline$-k_{x} \sin k_{x} L$ & $\cos k_{x} L$ & 0 & 0 & 0 & $\frac{h}{k_{x}} \sin k_{x} L$ \\
\hline 0 & 0 & $\cos k_{y} L$ & $\frac{1}{k_{y}} \sin k_{y} L$ & 0 & 0 \\
\hline 0 & 0 & $-k_{y} \sin k_{y} L$ & $\cos k_{y} L$ & 0 & 0 \\
\hline$-\frac{h}{k_{x}} \sin k_{x} L$ & $-\frac{h}{k_{x}^{2}}\left(1-\cos k_{x} L\right)$ & 0 & 0 & 1 & $-\frac{h^{2}}{k_{x}^{3}}\left(k_{x} L-\sin k_{x} L\right)$ \\
\hline 0 & 0 & 0 & 0 & 0 & 1 \\
\hline
\end{tabular}

Deinitions: $\mathrm{k}=1 / \rho_{0}, \mathrm{k}_{\mathrm{x}}^{2}=(1-\mathrm{n}) \mathrm{h}^{2}, \mathrm{k}_{\mathrm{y}}^{2}=\mathrm{nh}^{2}$

$$
\begin{aligned}
& a=h L=\text { the angle of bend } \\
& L=\text { path length of the central trajectory. }
\end{aligned}
$$


The field expansion for the midplane of a bending magnet is taken from Eq. (18) page 31 of SLAC-75, thereby defining the dimensionless quantities $\mathrm{n}$ and $\beta$ as follows:

$$
B_{y}(x, 0, t)=B_{y}(0,0, t)\left[1-n h x+B h^{2} x^{2}+\gamma h^{3} x^{3}+\ldots\right] \text {. }
$$

The type code signifying a BEND is 4.0. The input format for a TRANSPORT calculation is:

$$
\text { 4. L. B. n. }, \sqrt{1} \text { Label (not to exceed } 4 \text { spaces) }
$$

If $\mathrm{n}$ is not included in the data entry, the program assumes it to be zero. A $\beta$ entry for a second-order calculation is made via the 16.01 .0 element. (Do not confuse this $B$ with a pole-face rotation.)

The standard units for $\mathrm{L}$ and $\mathrm{B}$ are metres and $\mathrm{kG}$. If desired, these units may be changed by 15.08 .0 and 15.09 .0 type code entries preceding the BEAM Card. 


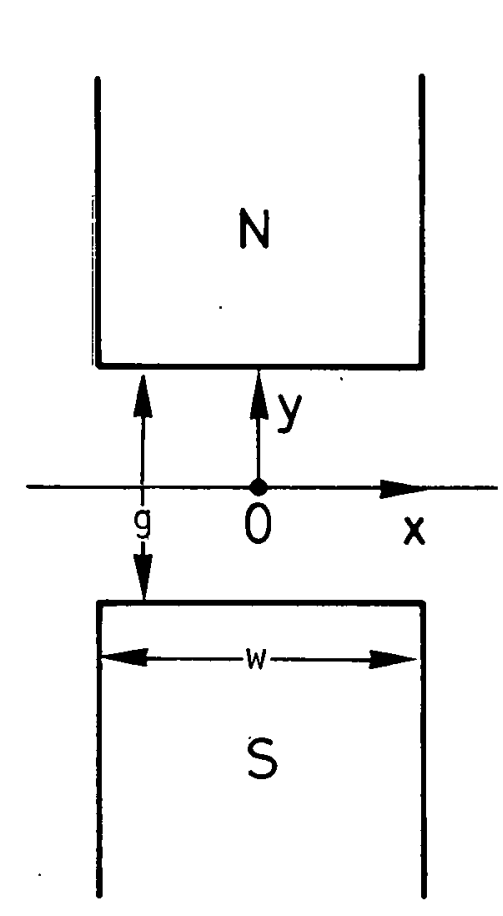

DIPOLE

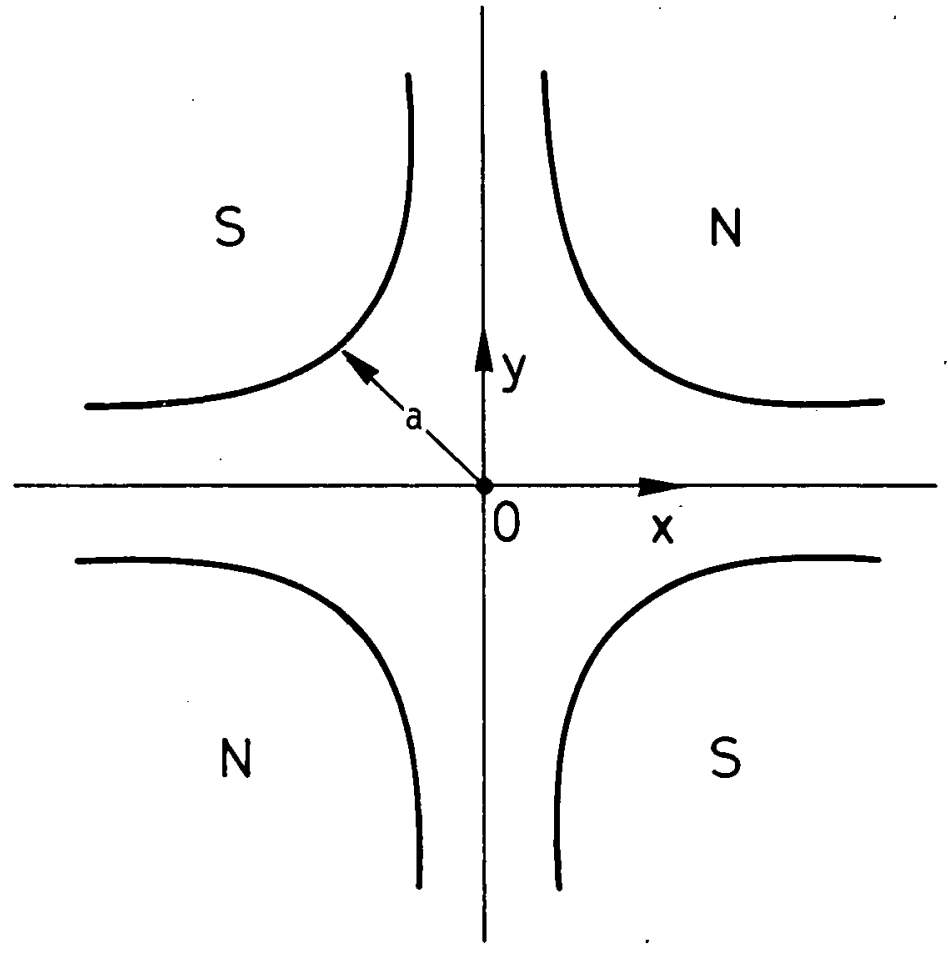

QUADRUPOLE

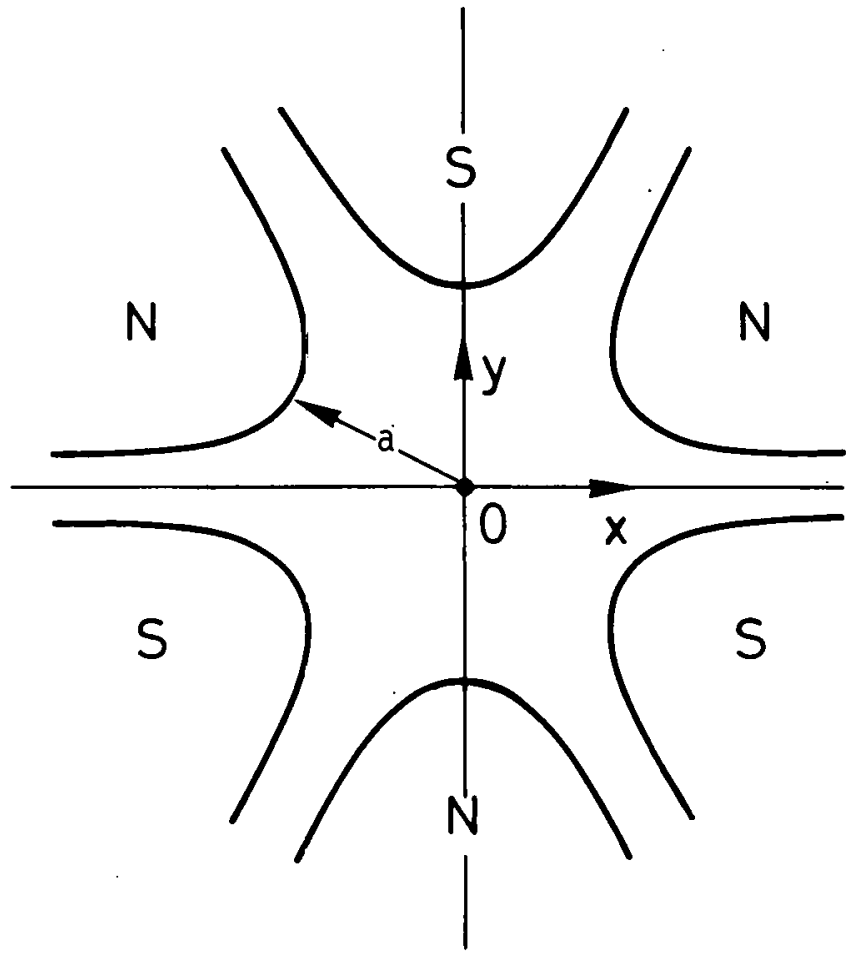

SEXTUPOLE

ILLUSTRATION OF THE MAGNETIC MIDPLANE ( $x$ AXIS) FOR DIPOLE, QUADRUPOLE AND SEXTUPOLE ELEMENTS. THE MAGNET POLARITIES INJICATE MULTIPOLE ELEMENTS THAT ARE POSITIVE WITH RESPECT TO EACH OTHER. 


\section{QUADRUPOLE: Type code 5.0}

A quadrupole provides focusing in one transverse plane and defocusing in the other.

There are four parameters to be specified for a TRANSPORT calculation:

1 - Type code 5.0 (specifying a quadrupole).

2 - (Effective) magnet length $L$ (in metres).

3 - Field at pole tip B (in $k G$ ). A positive field implies horizontal focusing; a negative field, vertical focusing.

4 - Half-aperture a (in $\mathrm{cm}$ ). Radius of the circle tangent to the pole tips.

The length and field of a quadrupole may be varied in first-order fitting. 'l'he aperture may not be.

The strength of the quadrupole is computed from its field, aperture and length. The horizontal focal length is printed in parentheses as output. A positive focal length indicates horizontal focusing and a negative focal length indicates horizontal defocusing. The quantity actually printed is the reciprocal of the $(\theta / x)$ transfer matrix element $\left(1 / R_{21}\right)$ for the quadrupoles. Thus two identical quadrupoles of opposite polarity will have different horizontal focal lengths due to the difference between the sine and the hyperbolic sine.

The type code for a QUAD is 5.0. The input format for a typical data set is:

Label (if desired) not to exceed 1 epacee between quotes

5. L. B. a. ' ' ;

The standard TRANSPORT units for $\mathrm{L}, \mathrm{B}$, and a are metres, $\mathrm{kG}$, and $\mathrm{cm}$, respectively. If other units are desired they must be chosen via the appropriate 15.0 type code entries preceding the BEAM (type code 1.0) card. 
First-order quadrupole matrix

\begin{tabular}{|c|c|c|c|c|c|}
\hline $\cos k_{q} L$ & $\frac{1}{k_{q}} \sin k_{q} L$ & 0 & 0 & 0 & 0 \\
\hline$-k_{q} \sin k_{q} L$ & $\cos k_{q} L$ & 0 & 0 & 0 & 0 \\
\hline 0 & 0 & $\cosh \mathrm{k}_{\mathrm{q}} \mathrm{L}$ & $\frac{1}{k_{q}} \sinh k_{q} L$ & 0 & 0 \\
\hline 0 & 0 & $k_{q} \sinh k_{q} L$ & $\cosh \mathrm{k}_{\mathrm{q}} \mathrm{L}$ & 0 & 0 \\
\hline 0 & 0 & 0 & 0 & 1. & 0 \\
\hline 0 & 0 & 0 & 0 & 0 & 1 \\
\hline
\end{tabular}

These elements are for a quadrupole which focuses in the horizontal ( $x$ ) plane (B positive). A vertically ( $y$-plane) focusing quadrupole (B negative) has the first two diagonal submatrices interchanged.

Definitions: $L=$ the effective length of the quadrupole

$$
\begin{aligned}
a= & \text { the radius of the aperture } \\
B_{0}= & \text { the field at radius } a \\
k_{q}^{2}= & \left(B_{0} / a\right)\left(1 / B \rho_{0}\right) \text {, where }\left(B \rho_{0}\right)=\text { the magnetic rigidity } \\
& \text { (momentum) of the central trajectory. }
\end{aligned}
$$




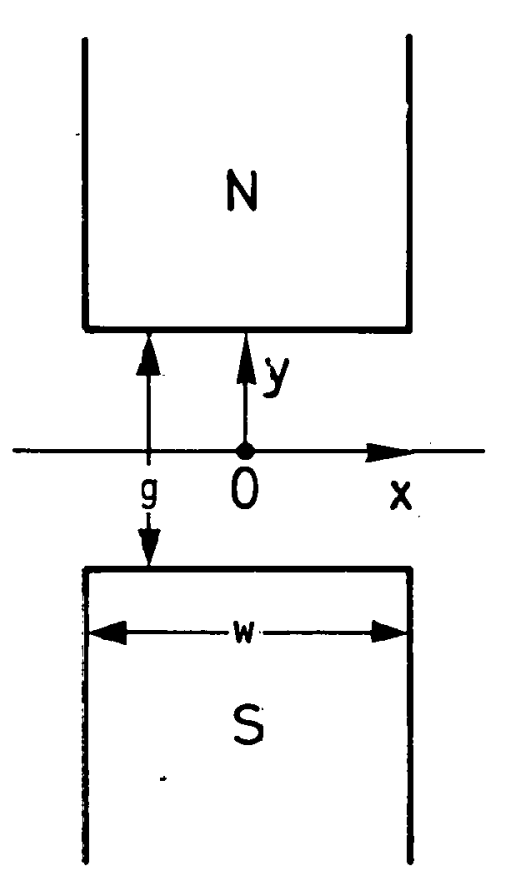

DIPOLE

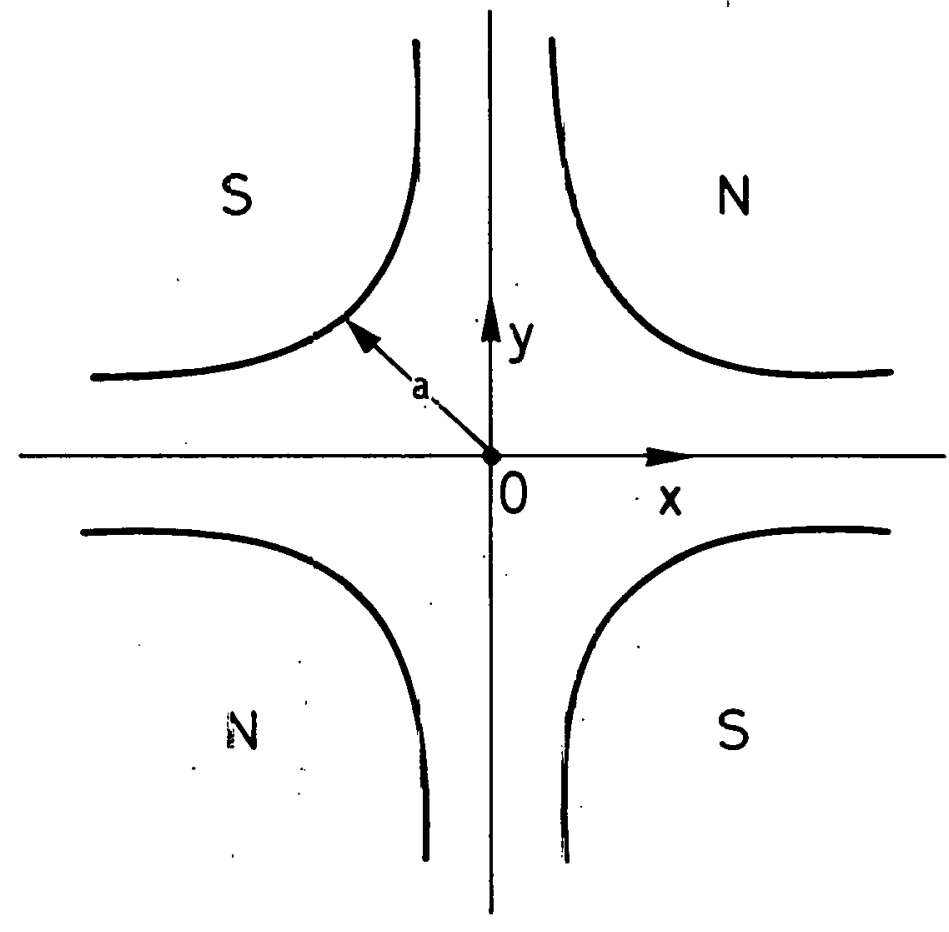

QUADRUPOLE

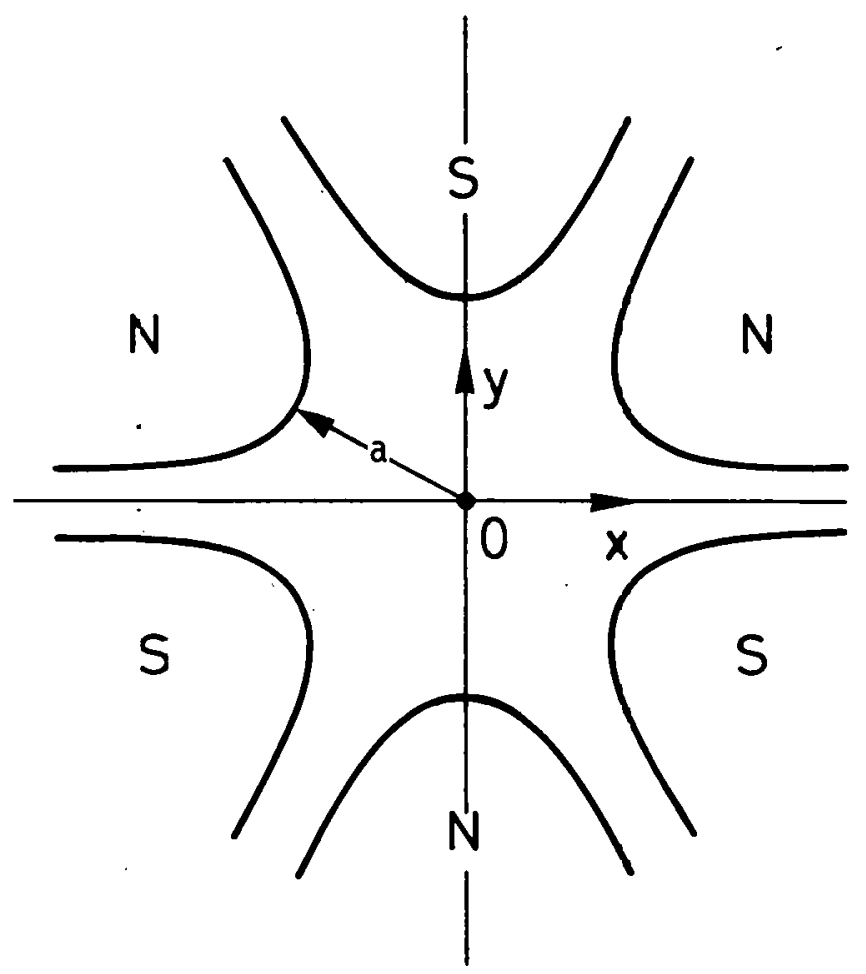

SEXTUPOLE

ILLUSTRATION DF THE MAGMETIC MIDPLANIE ( $X$ AXIS) FOR DIPOLE, QUADRUPO־E ANU SEXTUPOLE ELEMENTS. THE MAGNET POLARITIES INDICATE MULT_POLE ELEMENTS THAT ARE POSITIVE ЙITH RESPECT TO EACH OTHER. 
TRANSFORM 1 update $^{\star}$ ): Type code 6. 0. 1.

To re-initialize the matrix TRANSFORM 1 (the product of the $R$ matrices, R1) use type code 6.0. A (6, 0.1. ;) card effects an update of the Rl matrix and initiates the accumulation of a new product matrix at the point of the update. This facility is of ten useful for misaligning a set of magnets or fitting only a portion of a system.

The matrix Rl is updated by no other element. It is not used in the calculation of the beam matrix. The beam matrix is calculated from the auxiliary transfer matrix R2 described on the next page.

A TRANSFORM 1 matrix will be printed at any position in the data set where a (13. 4. ;) entry is inserted.

See the following section for the introduction of an auxiliary transformation matrix R2 (TRANSFORM 2) to avoid the need for TRANSFORM 1 updates.

The (6. 0. 1. ;) card also causes an update of the R2 matrix.

*) By "updating" we mean initiating a new starting point for the accumulation (multiplication) of the $R$ matrix. At the point of update the previous accumulation is discontinued. When the next element possessing a transfer matrix is encountered, the accumulated transfer matrix $\mathrm{Rl}$ is set equal to the individual transfer matrix $\mathrm{R}$ for that element. Accumulation is resumed thereafter. 


\section{AUXILIARY TRANSFORMATION MATRIX (R2): Type code 6.0.2.}

The R1 matrix represents the accumulated transfer matrix from either the beginning of the beam line or the last explicit RI update (6. 0. 1. ;). However several elements in TRANSPORT which affect the beam matrix cannot be represented in any transfer matrix. To avoid update complications with R1 an auxiliary transfer matrix R2 exists. The beam matrix is then calculated from the R2 matrix and the beam matrix at the last R2 update.

Both the R1 and R2 matrices are normally available for printing. However there is no redundancy in computer use, since, internally to the program only R2 is calculated at each element. The matrix R1 is calculated from R2 on1y as needed.

The R2 matrix is updated explicitly via a (6. 0. 2, ;) entry. It may be printed by a (13. 24. ;) entry. Constraints on R2 are imposed similarly to those on R1. For details see the section describing type code 10.0

The complete list of elements which update TRANSFORM 2 is:

1) a beam type code 1.0 entry

2) the (6.0.1.;) entry

3) the (6, 0, 2. ;) entry

4) a centrold shift type code 7.0 entry

5) a misalignment type code 8.0 entry

6) a stray field type code 21.0 entry. 
SHIFT IN THE BEAM CENTROID: Type code 7.0

Sometimes it is convenient to redefine the beam centroid ${ }^{* *}$ ) such that it does not coincide with the TRANSPORT reference trajectory. Provision has been made for this possibility via type code 7.0. Seven parameters are required:

$$
1 \text { - Type code } 7.0 .
$$

( 2 to 7 ) - the coordinates $x, \theta, y, \phi, l$, and $\delta$ defining the shift in the location of the beam centroid with respect to its. previous position. The units for $\mathrm{x}, \theta, \mathrm{y}, \phi, \ell, \delta$ are the same as those chosen for the BEAM (type code 1.0 entry), normally $\mathrm{cm}$, $\mathrm{mr}, \mathrm{cm}, \mathrm{mr}, \mathrm{cm}$, and percent.

The transformation matrix $\mathrm{R} 2$ is updated by this element.

In order for this code to function properly, the initial BEAM entry (type code 1.0 ) must have a non-zero phase space volume, for example a

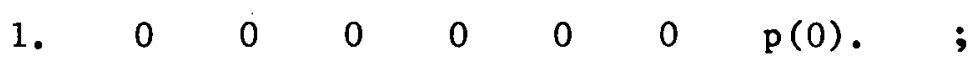

BEAM entry is not permissible when calculating a shift in the beam centroid; whereas a

$$
\text { 1. } 1.1 .1 .1 .1 .10 \text {. } 1.10) \text {; }
$$
entry (non-zero phase volume) is acceptable.

**) By "beam centroid" we mean the centre of the beam ellipsoid. 
MAGNET AL IGNMENT TOLERANCES: TyPe code 8.0

To first order the misalignment of a magnet affects only the centroid of the beam. Two varieties of misalignment are commonly encountered: the magnet is displaced and/or rotated by a known amount; or the actual position of the magnet is uncertain within a given tolerance. Either of these situations may be simulated through use of the 'align' element.

There are eight parameters to be specified:

1 - Type code 8.0 (specifying alignment).

2 - The magnet displacement in the horizontal direction $(\mathrm{cm})$.

3 - A rotation about the horizontal axis (mr).

4 - A displacement in the vertical direction $(\mathrm{cm})$.

5 - A rotation ahnut the vertical axis $(m x)$.

6 - A displacement in the beam direction $(\mathrm{cm})$.

7 - A rotation about the beam direction ( $m r$ ).

8 - A three digit code number (defined below) specifying the type of misalignment.

The coordinate system employed is that to which the beam is referred at the point it enters the magnet. For example, a rotation of a bending magnet about the beam direction (parameter 7 above) is referred to the direction of the beam where it enters the magnet. The units employed are the standard TRANSPORT units shown above, unless redefined by type code 15.0 entries. If the units are changed, the units of the misalignment displacements are those determined by the 15. 1. type code entry; the units for the misalignment rotations are those determined by the 15. 2. type code entry.

The misalignment of any physical element may be simulated. A beam line rotation (type code 20.0 ) may be included in a misaligned section. Thus one can simulate the misalignment of magnets that bend vertically. The arbitrary matrix (type code 14.0) may not be included in a misaligned section. The type code 8.0 align element(s) must directly follow the magnetic element(s) to be misaligned.

When the actual position of the magnet(s) is uncertain within a given tolerance, the BEAM card entry at the beginning of the system should correspond to a zero phase space input, i.e. the BEAM card should appear as follows: 
1. . 0. 0. $0 . \quad 0 . \quad 0 . \quad 0 . \quad \mathrm{p}(0) . ; \quad$;

The phase ellipse print-outs following the misalignment will then possess a non-zero phase volume which-represents the uncertainty in the position of the beam centroid resulting from the uncertainty in the knowledge of the positioning of the magnet(s).

When the misalignment is a known amount, the BEAM card entry at the beginning of the system should possess a non-zero phase volume (for example a

$$
\text { 1. 1. 1. 1. 1. 1. 1. } 1 . \text { p (0). ; }
$$

is a permissible type code 1.0 entry).

The beam centroid print-outs following the misalignment will then show a definite shift in the position of the beam centroid resulting from the misalignment of the magnet(s). 
An align element updates the BEAM (sigma) matrix and the R2 matrix but not the R1 matrix.

The following three digit code numbers provide several alternative misalignment situations:

A. The units position specifies the section of the beam line to be misaligned.

\begin{tabular}{|c|c|}
\hline $\begin{array}{c}\text { CODE } \\
\text { NUMBER } \\
\end{array}$ & INTERPRETATION \\
\hline xxo. & $\begin{array}{l}\text { In this case, the } 0 \text { means that the information contained on } \\
\text { the align card refers only to the single magnet (type code } \\
\text { entry) immediately preceding it. }\end{array}$ \\
\hline $\mathrm{xx} 1$. & $\begin{array}{l}\text { With this option the } 1 \text { means that all of the type code } \\
\text { elements since the last R } 1 \text { matrix update (the start of the } \\
\text { beam line or a } 6.0 .1 \text {. ; type code entry) are treated as a } \\
\text { unit and the misalignment information on the card is applied } \\
\text { to the unit as a whole. This code digit, for example, is } \\
\text { necessary for studying misalignment of quadrupole triplets } \\
\text { and other combinations involving more than two quadrupole } \\
\text { magnets. }\end{array}$ \\
\hline $\mathrm{xx} 2$. & $\begin{array}{l}\text { With this option the } 2 \text { means that all of the type code } \\
\text { elements since the last R2 matrix update (see type code } 6.0 \\
\text { for a list of olemento which update } R 2 \text { ) are treated as a } \\
\text { unit. It makes use of the fact that R2 matrix updates do } \\
\text { not affect the } R 1 \text { matrix. }\end{array}$ \\
\hline & $\begin{array}{l}\text { This option should be used for the misalignment of a bending } \\
\text { magnet when fringing fields or pole face rotations (type } \\
\text { code } 2.0 \text { ) are included. See examples } 1 \text { and } 2 \text { below for an } \\
\text { illustration of this. Another example of the use of this } \\
\text { option is in the misalignment of an array of quadrupoles. } \\
\text { By successive application of align elements, for example, } \\
\text { the elements of a quadrupole doublet could be misaligned } \\
\text { relative to each other and then the doublet as a whole could } \\
\text { be misaligned. }\end{array}$ \\
\hline
\end{tabular}


B. The tens position defines the system axis along which the succeeding magnets are positioned:

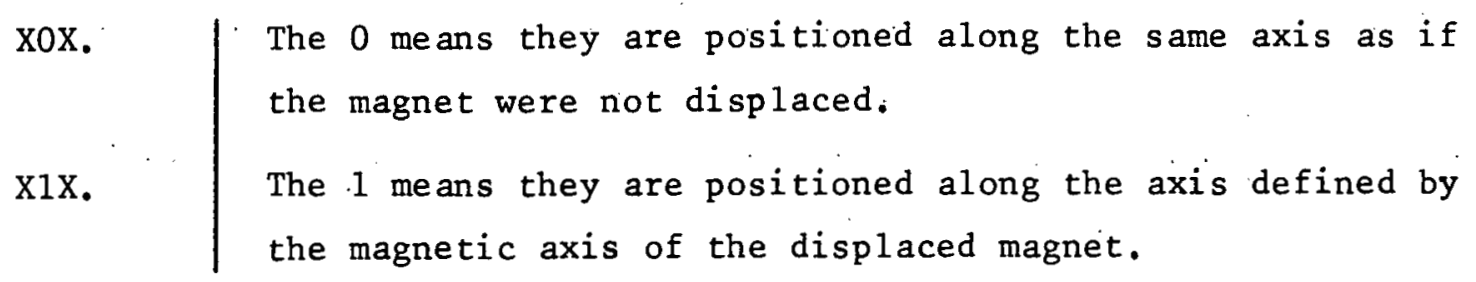

For instance, if a quadrupole is displaced, the remainder of the system may be left alone (XOX.) or displaced with the quadrupole (X1X.).

C. The hundreds position distinguishes between an uncertainty in position ( $\left.\mathrm{XXX}_{\bullet}\right)$ or a known displacement ( $\left.1 \mathrm{XX}_{*}\right)$.

Any combination of digits may be used to define the exact circumstances intended. Thus, code 111. $(=1 .+10 .+100$.$) indicates the$ deliberate displacement of a set of magnets and the remainder of the system (referred to the point the beam enters the set). Code 101. would leave the remainder of the system unaltered. 
Typical code numbers for magnet alignment tolerances might be:

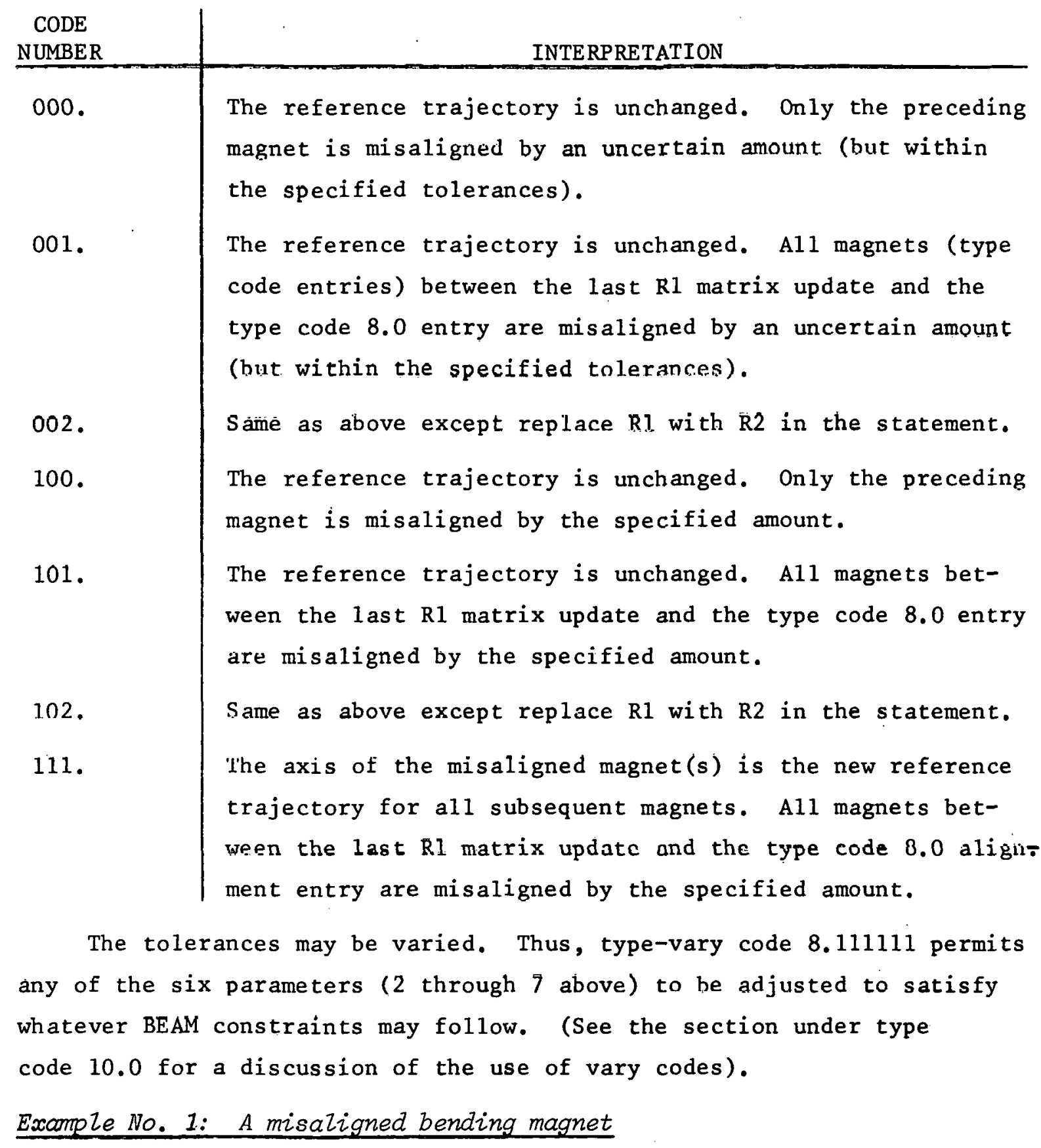

A bending magnet (including fringing fields) misaligned by a known amount might be represented as follows:

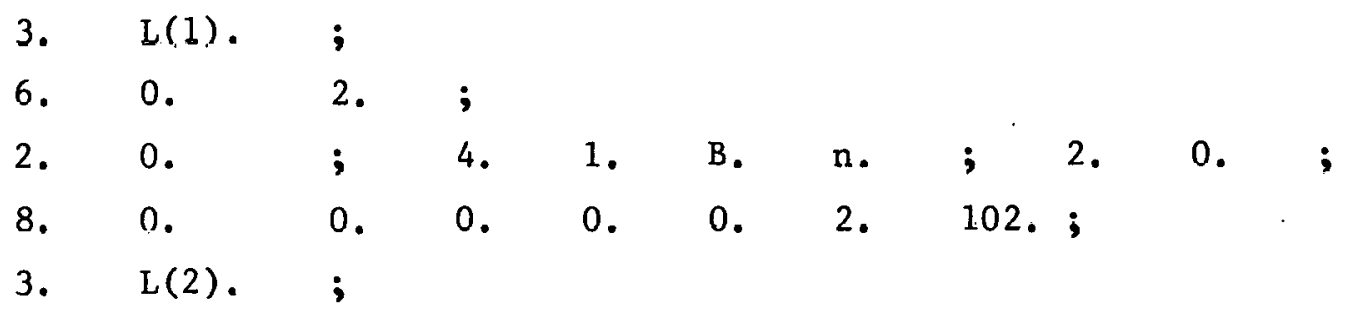


This represents a known rotation of the bending magnet about the incoming beam direction ( $z$ axis) by $2.0 \mathrm{mr}$. The result of this misalignment will be a definite shift in the beam centroid. The 6. 0.2.; transform 2 update is necessary because of the use of the 2. 0 . ; entries before and after the bending magnet. The code number XX2. is required because the magnetic array (bending magnet + fringing fields) consists of three type code elements instead of one. Example No. 2: A misaligned bending magnet

A bending magnet having an uncertainty of $2 \mathrm{mrad}$ in its angular positioning about the incoming beam ( $z$ axis) would be represented as follows :

3. $\mathrm{L}(1)$;

6. $0 . \quad$;

2. $0 . \quad$;. L. B. n. ; 2. 0. ;

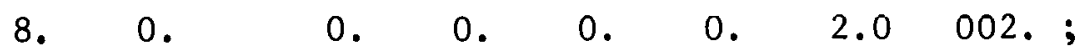

3. L(2). ;

The result of this misalignment will be an uncertainty in the location of the outcoming beam centroid -- hence to observe this, the input BEAM card should have zero phase space dimensions as follows:

1. $\quad$ 0. $0 . \quad$ o. $\quad$ o. $\quad$ o. $\quad$ o. p $(0)$; Example No. 3: A misaligned quadrupole triplet

One typical use of both the R1 and R2 matrices is to permit the misalignment of a triplet. For example, an uncertainty in the positions within the following triplet:

5. $1 . \quad-8 . \quad 10 . \quad$;

5. 2. $\quad+7$. 10 ;

5. 1. $\quad$-8. 10 .

may be induced by appropriate 8 . clemente as noted: 
6. $\quad 0 . \quad 1 . \quad$;

5. 1. $\quad-8$. 10. ;

6. 0.2 . ;

5. 2. +7. 10. ;

5. 1. -8 . 10 . ;

8. - - - - - -

8. - - - - - - - - - -

8. -- -- -- -- - - -

The first 8.0 card in the list refers to misalignment of the third magnet only. The second 8.0 refers to misalignment of the second and third magnets as a unit via the R'2 matrix update (the 6. 0, 2, ; entry). The last 8. refers to misalignment of the whole triplet as a unit via the R1 matrix update (the 6. 0. 1. ; entry),

The BEAM card (type code 1.0 entry) should appear as shown in Example 2 above. 
REPETITION: Type code 9.0

Many systems include a set of elements that are repeated several times. To minimize the chore of input preparation, a 'repeat' facility is provided.

There are two parameters:

1 - Type code 9.0

2 - Code dıgle. If non-zero, it states the number of repetitions desired from the point it appears. If zero it marks the end of a repeating unit.

For example, a total bend of 12 degrees composed of four 3-degree bending magnets each separated by 0.5 metres could be represented by 9. 4.; 4. -- ; 3. .5; 9. 0.; Those elements (in this case a. bend and drift) between the 9. 4.; and 9.0.; would be employed four times.

There is no indication of the 9.0 cards in the printed TRANSPORT output when calculating except for the repeated listing of the elements they control.

Vary codes may be used within a repeating unit in the usual fashion. However all repetitions of a given varied element will be coupled.

Repeat cards may be nested four deep. By "nesting" we mean a repeat within a repeat. An example is given below. 
Example of Nesting

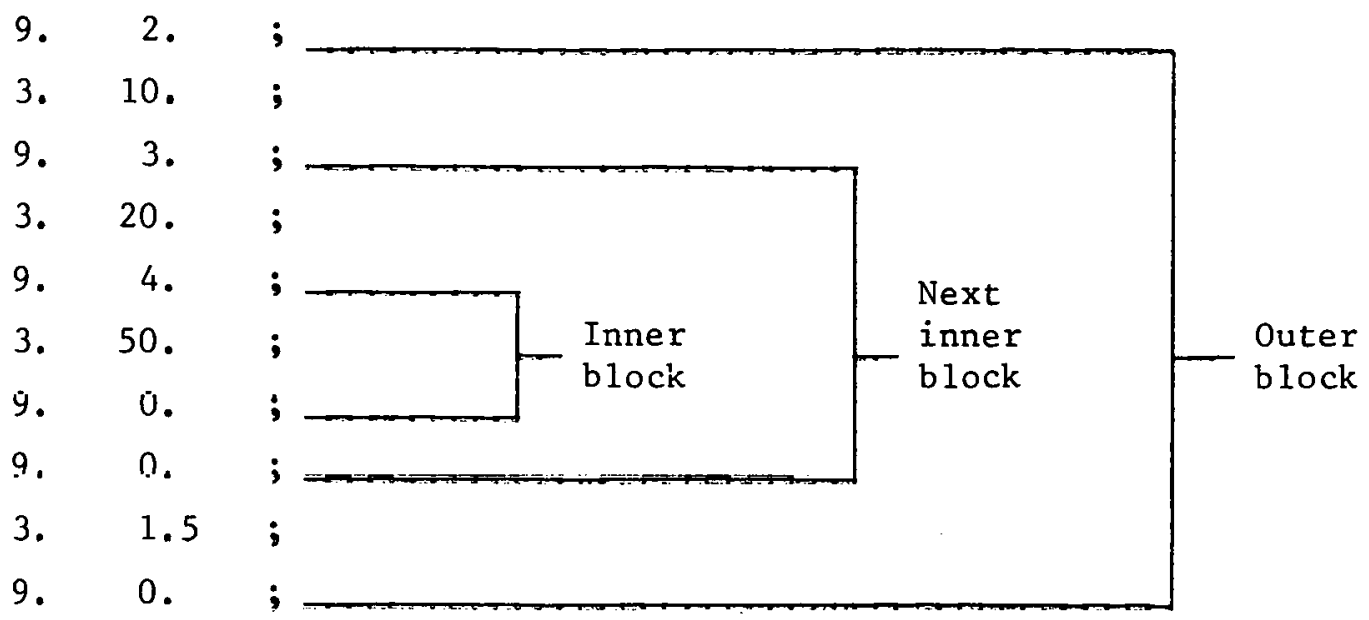

The total length of this sequence is:

$2 *(10 .+3 *(20+4 * 50)+1.5)=1343$. 
VARY CODES and FITTING CONSTRAINTS: Type code 10.0

Some (not all) of the physical parameters of the elements comprising a beam line may be varied in order to fit selected matrix elements. In a first-order calculation one might fit elements of the R1 or R2 transformation matrices or the beam (sigma) matrix. In second order one might constrain an element of the second-order matrix Tl or minimize the net contribution of aberrations to a given beam coordinate. Special constraints are also available.

One may not mix orders in fitting. First order vary codes and constraints must be inserted only in a first-order calculation, and similarly for second order.

The physical parameters to be varied are selected via 'Vary Codes' attached to the type codes of the elements comprising the system. The fitting constraints on matrix elements are selected via type code 10.0 entries placed in the system where the constraint is to be imposed. Vary codes

Associated with each physical element in a system is a vary code which specifies which physical parameters of the element may be varied. This code occupies the fraction portion of the type code specifying the element. It has one digit for each parameter, the digits having the same order in the code as the physical parameters have on the card. A ' $O$ ' indicates the parameter may not be varied; $a$ ' $I$ ' that it may be. For instance, 3.0 is the combined type (3) and vary code (0) for a drift length whish is to remain fixed; 3.1 indicates a drift length that may be varied (by the virtue of the .1). The type code 4.010 indicates a bending magnet with a variable magnetic field. In punching the code 3.0, the zero need not be punched. In punching the 4.010 code, the first zero must be punched but the second zero need not be. 


\section{Eirst-order vary codes}

In a first-order run the following parameters marked $v$ may be varied, those marked 0 may not be varied.

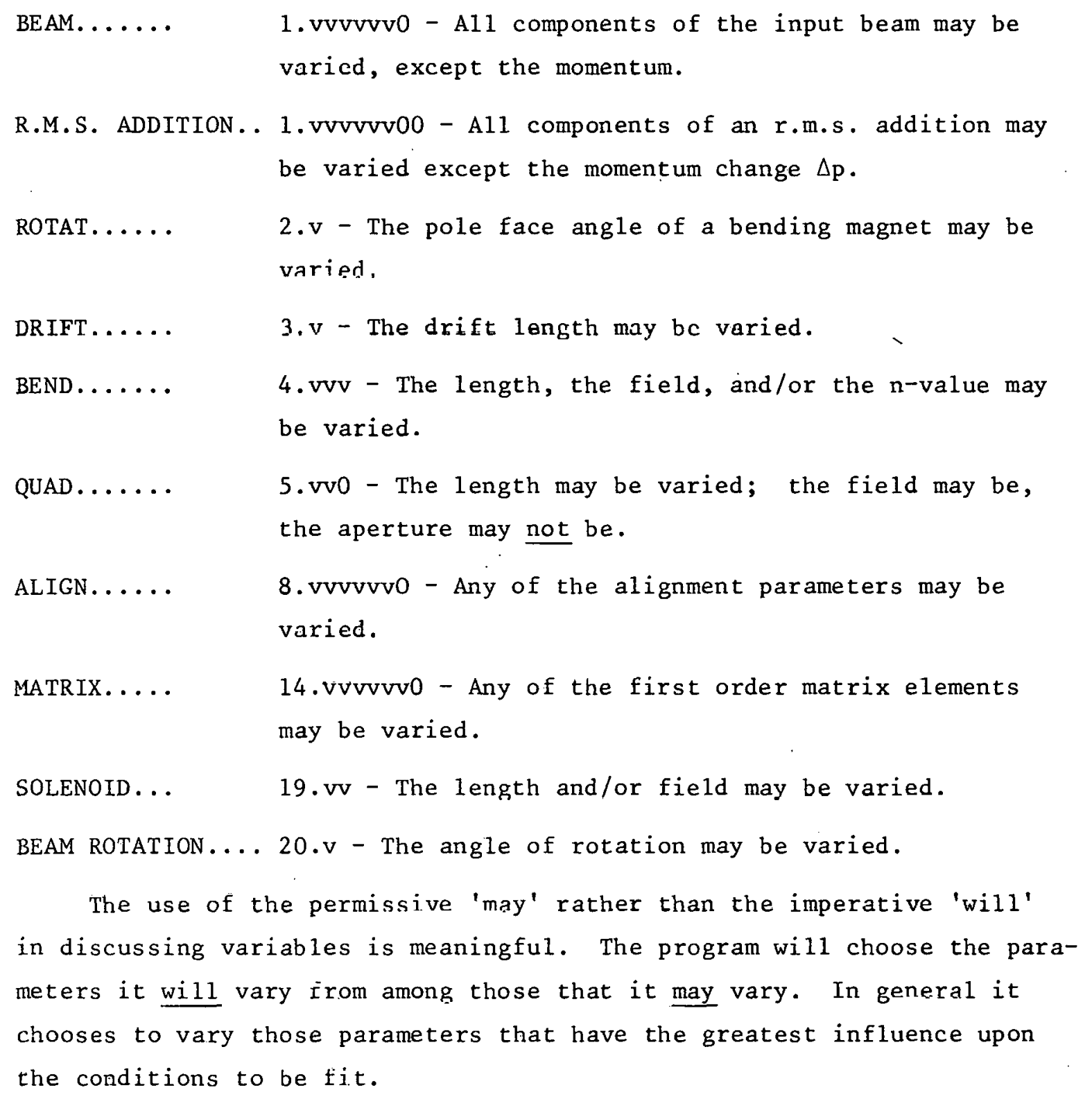


Second-order vary codes

In a second-order run the following parameters may be varied:

DRIFT.... 3.v - The drift length may be varied. Variation of a drift length should be done with zaution as it may affect the first-order properties of the beam line. But inversely coupled drift spaces straddling a sextupole will, for example, show only second-order effects.

$\varepsilon(1) \ldots . .16 .0 \mathrm{~V}$ 1. - The normalized quadratic term (sextupole component) in the midplane expansion for the field of a bending magnet may be varied.

1/R1.... 16.0v 12. - The pole face curvature of a bending magnet entrance may be varied.

1/R2..... 16.0v 13. - The pole face curvature of a bending magnet exit may be varied.

SEXTUPOLE $18.0 \mathrm{v}$ - The field strength may be varied.

The special parameter cards (type code 16.0) once introduced apply to all subsequent magnets in a beam line until another type code 16.0 specifying the same parameter is encountered. Thus if such a parameter is varied, the variation will apply simultaneously to all subsequent magnets to which it pertains. The variation will persist until the parameter or vary code attached to the parameter is changed by the introduction of another type code 16.0 card specifying the same parameter. 


\section{Coupled vary codes}

It is possible to apply the same correction to each of several variables. This may be done by replacing the digit 1 in the vary code with one of the digits 2 through 9, or a letter A through $Z$. All such variables whosc vary digits are the same, regardless of position will receive the-same correction. For example, the three type-vary codes $(5.0 \mathrm{~A}, 5.01,5.0 \mathrm{~A})$ might represent a symmetric triplet. The same correction will be made to the first and third quadrupoles, guaranteeing that the triplet will remain symmetric.

If a vary digit is immediately preceded by a minus sign, the computed correction will be subtracted from, rather than added to, this variable. Thus parameters with the same vary digit, one of them being preceded by a minus sign, will be inversely coupled. For example the type-vary code sequence $(3 . B, 5.01,3 .-B)$ will allow the quadrupnle to move withnut altering the total system length.

Vary digits may also be immediately preceded by a plus sign without changing their meaning. Thus 5.0A is the same as 5.0+A. For historical reasons, the vary digits (9 and 4$),(8$ and 3$)$, and $(7$ and 2$)$ are also inversely coupled. Inverse coupling: may not be used with type codes 1.0 or 8.0 .

The total number of independent variables in a first-order run is limited to 20 by reasons of: the mathematical method of fitting and to 10 for a second-order run. So tar as this limit is concerned, variables. that are tied together count as one. Variables within repeat elements (type code 9.0 ) also count only one. 


\section{Possible fitting constraints}

A variety of possible constraints is available. Fitting may be done in either first- or second-order, but not in both simultaneously. The order of the constraint must be appropriate to the order of the run. A list of constraints available is given below. They are explained more fully on later pages.

\section{First-order constraints}

1) An element of the first-order transfer matrix $R 1$.

2) An element of the auxiliary first-order matrix R2.

3) A $\sigma$ (BEAM) matrix element.

4) The correlations $r$ in the beam coordinates.

5) The first moments of the beam.

6) The total system length.

7) An AGS machine constraint.

\section{Second-order constraints}

1) An element of the second-order transfer matrix T1.

2) An element of the second-order auxiliary transfer matrix T2.

3) The net contributions of aberrations to a given coordinate of the beam matrix $\sigma$.

4) The strength of sextupoles used in the system.

The second-order matrices are actually computed using the auxiliary matrix T2. Therefore, when activating second-order fitting, one must not include any element which causes an update of the R2 matrix. For a complete 1 ist of such elements see type code 6.0 .

The present value of the constrained quantity, as well as the desired value, is printed in the output. In the case of transfer matrix elements this value may be checked by printing the transfer matrix itself. Certain other constrained quantities may be checked similarly. Exceptions are noted in the explanations following. 


\section{R1 matrix fitting constraints}

There are five parameters to be specified when imposing a constraint upon the (i, $j$ ) element of an R1 matrix.

1 - Type code 10. n (specifying that a fitting constraint follows).

2 - Code digit $(-i)$.

3 - Code digit $(j)$.

4 - Desired value of the ( $i, j$ ) matrix element.

5 - Desired accuracy of fit (standard deviation).

Note that any fitting constraint on an RI matrix element is from the preceding update of the RI matrix. An RI matrix is updated only by a (6. 0.1. ;) entry.

The symbol ( $n$ ) is normally zero or blank. If $n=1$, then entry 4 is taken to be a lower limit on the matrix element. If $n=2$, entry 4 is taken to be an upper limit. 
Some typical R1 matrix constraints are as follows:

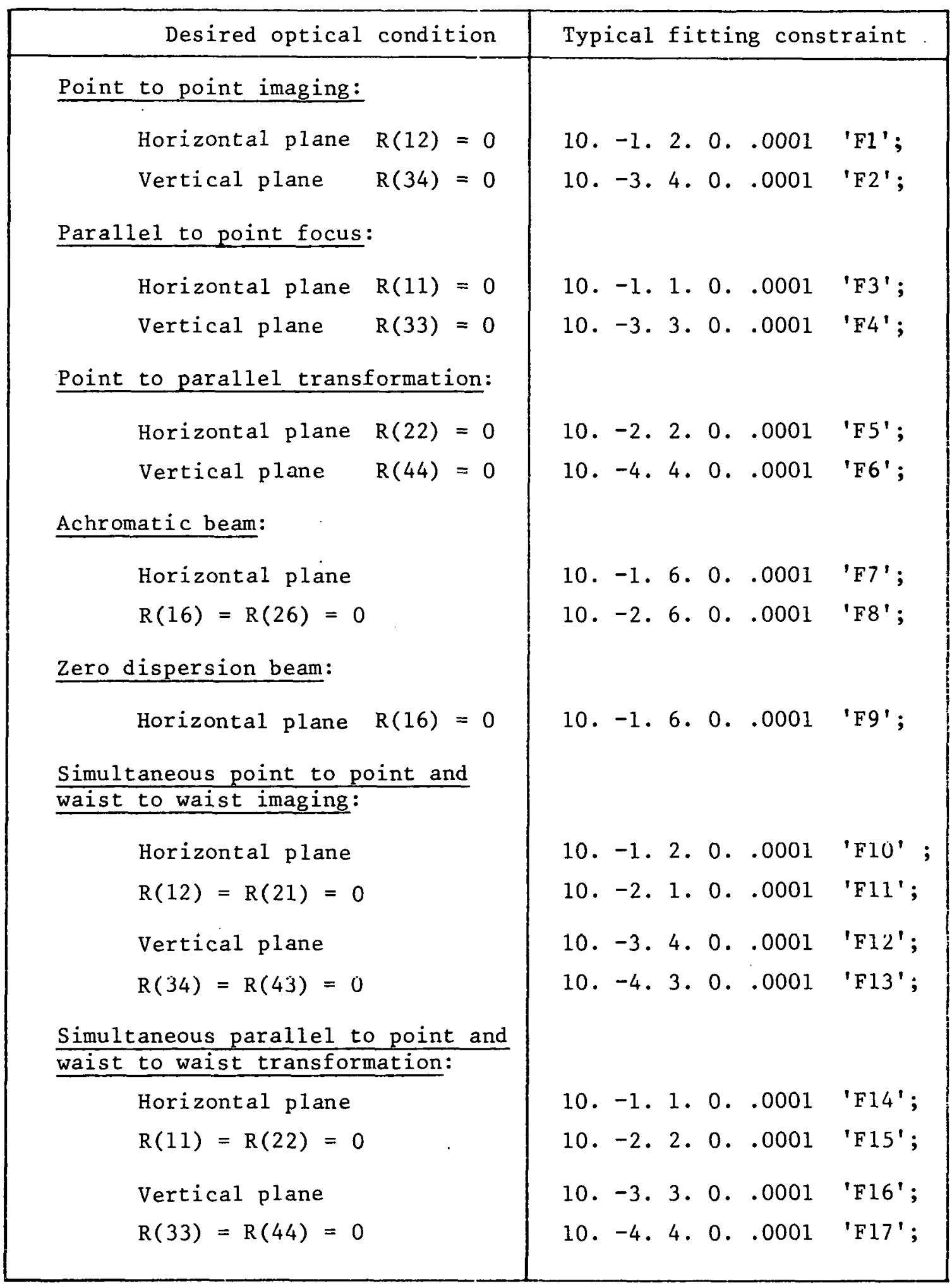


R2 matrix fitting constraints

There are five parameters to be specified when imposing a constraint upon the ( $i, j$ ) element of an R2 matrix.

1 - Type code $10 . \mathrm{n}$

2 - Code digit $-(20+i)$.

3 - Code digit $(\mathrm{j})$.

4 - Desired value of the $(i, j)$ matrix element.

5 - Desired accuracy of fit (standard deviation).

Some typical R2 matrix constraints are as follows:

The symbol (n) is normally zero or blank. If $n=1$, then entry $h$ is taken to be a lower limit on the matrix element. If $n=2$, entry 4 is taken to be an upper limit.

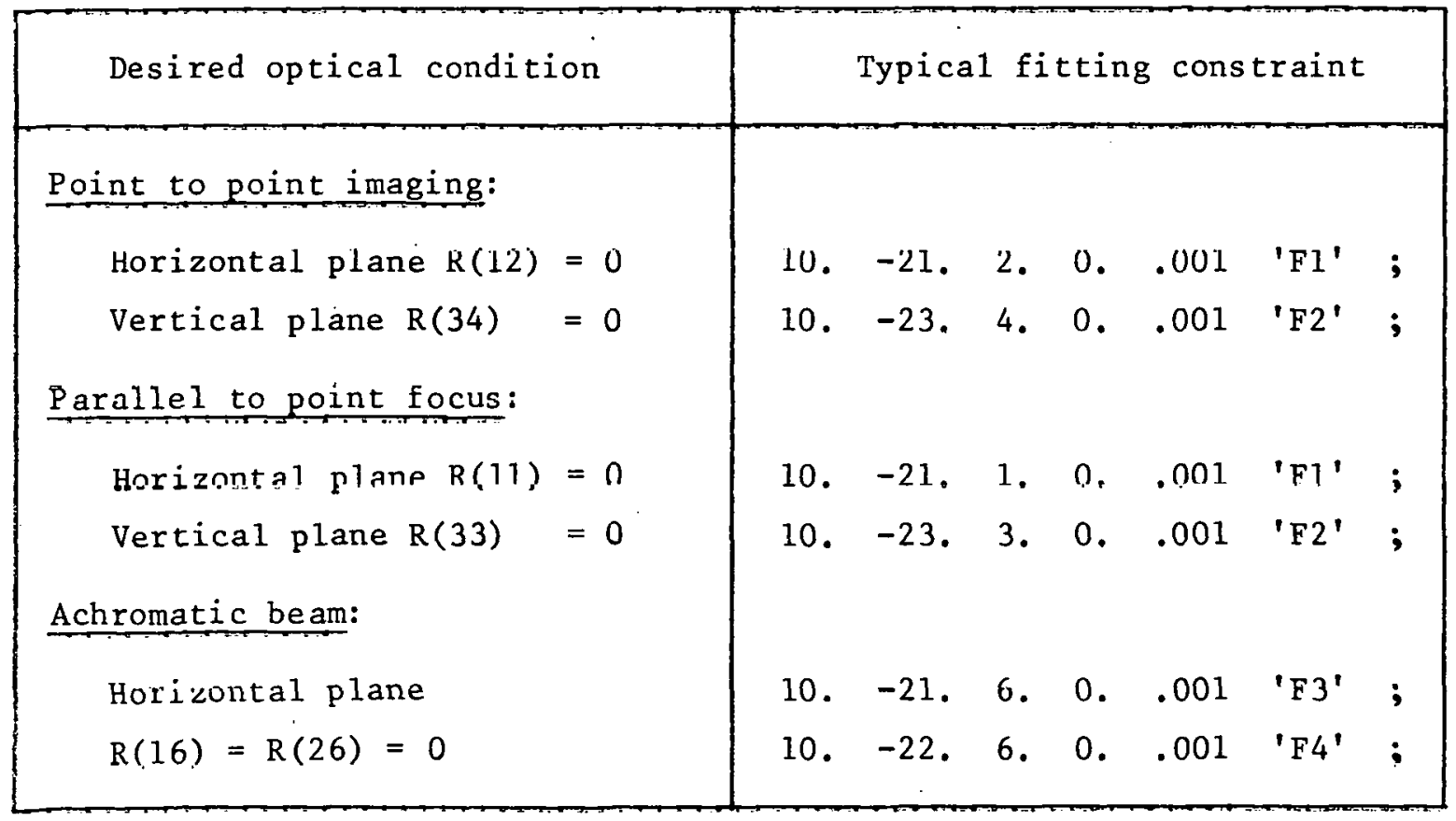

See type code 6.0 for a complete list of elements which update the R2 matrix. 
$\sigma(B E A M)$ matrix fitting constraints

There are five parameters to be specified when imposing a constraint upon the $(i, j)$ element of a $\sigma$ (BEAM) matrix.

1 - Type code $10, \mathrm{n}$

2 - Code digit $(i)$. ( $i \geq j)$

3 - Code digit $(j)$.

4 - Desired value of the $(i, j)$ matrix element.

5 - Desired accuracy of fit (standard deviation).

The symbol ( $\mathrm{n}$ ) is normally zero or blank. If $\mathrm{n}=1$, then entry 4 is taken to be a lower limit on the matrix element. If $n=2$, entry 4 is taken to be an upper limit. If $i=j$, then the value inserted in entry 4 is the desired beam size $(\sigma(i i))^{\frac{1}{2}}$ e.g. $x(\max )=(\sigma(11))^{\frac{1}{2}}$ etc.

Some typical $\sigma$ matrix constraints are as follows:

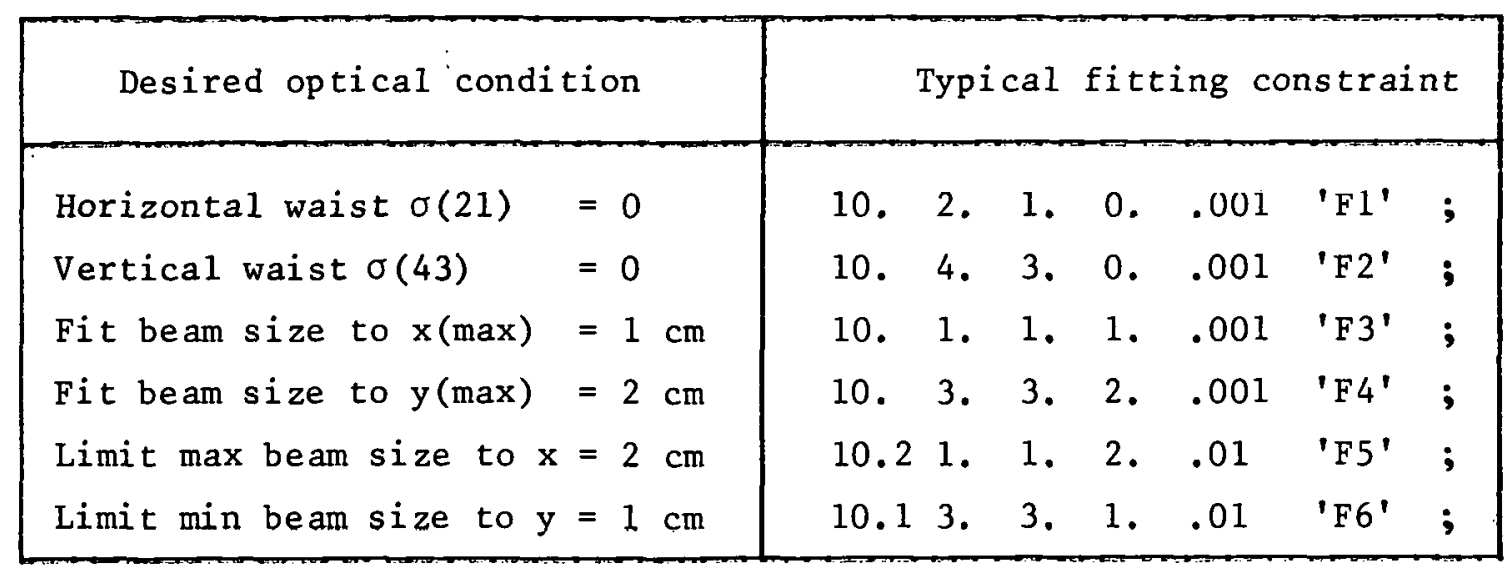

In general, it will be found that achieving a satisfactory 'beam' fit with TRANSPORT is more difficult than achieving an $K$ matrix fit. When difficulties are encountered, it is suggested that the user 'help' the program by employing sequential (step by step) fitting procedures when setting up the data for his problem. More often than not a "failure to fit" is caused by the user requesting the program to find a physically unrealizable solution. An often encountered example is a violation of Liouville's theorem. 


\section{Beam correlation matrix ( $r$ ) fitting constraints}

Five parameters are needed for a constraint on the (i, $j$ ) element of the beam correlation matrix.

$$
\begin{aligned}
& 1 \text { - lype code } 10 . n \\
& 2 \text { - Code digit }(10+i) . \\
& 3 \text { - Code digit }(j) \text {. } \\
& 4 \text { - Desired value of the }(i, j) \text { matrix element. } \\
& 5 \text { - Desired accuracy of fit (standard deviation). }
\end{aligned}
$$

TRANSPORT does not print the beam $(\sigma)$ matrix directly. Instead it prints the bcam half widths and represents the off-diagonal elements by the correlation matrix. If one wishes to fit an element nf this matrix to a non-zero value it is convenient to be able to constrain the matrix element directly.

$$
\text { Some typical } \mathrm{r} \text { matrix constraints are as follows: }
$$

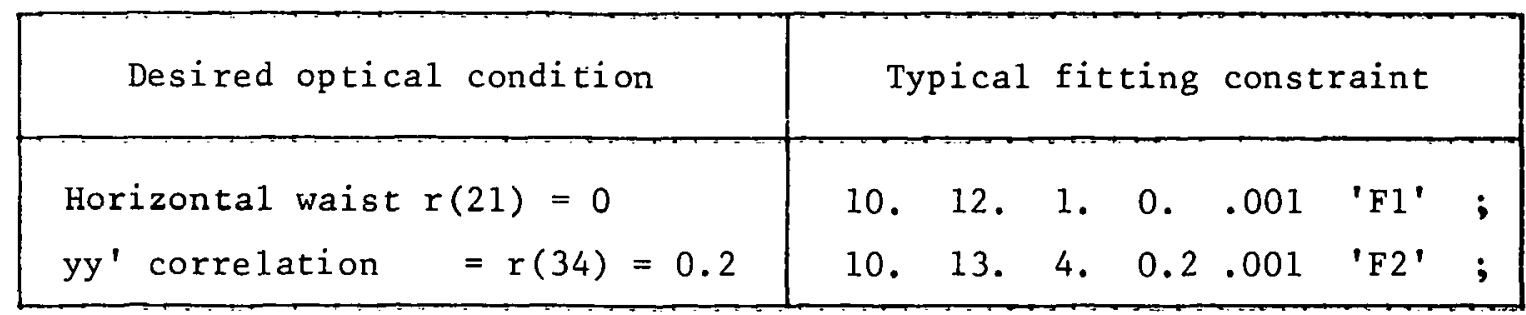




\section{First moment constraint}

In first order, known misalignments and centroid shifts cause the centre (centroid) of the phase ellipsoid to be shifted from the reference trajectory, i.e., they cause the beam to have a non-zero first moment. The first moments appear in a vertical array to the left of the vertical array giving the $\sqrt{\sigma(i i)}$. The units of the corresponding quantities are the same.

It is perhaps helpful to emphasize that the origin always lies on the reference trajectory. First moments refer to this origin. However, the ellipsoid is defined with respect to its centre, so the covariance matrix, as printed, defines the second moment about the mean.

First moments may be fitted. The code digits are $i=0$ and $j$, where $j$ is the index of the quantity being fit. Thus 10. 0.1. .1.01; constrains the horizontal (1.) displacement of the ellipsoid to be $0.1 \pm 0.01 \mathrm{~cm}$.

This constraint is useful in deriving the alignment tolerances of a system or in warning the system designer to offset the element in order to accommodate a centroid shift. 
System length constraint

A running total of the lengths of the various elements encountered is kept by the program and may be fit. The code digits are $i=0$, $\mathbf{j}=0$.

Thus the element (10.0.0.150,5.;) would make the 'length of the system prior to this element equal to $150 \pm 5$ metres. Presumably there would be a variable drift length somewhere in the system. By redefining the cumulative length via the (16.6. L. ;) element, partial system lengths may be accumulated and fit. 
AGS machine constraint ${ }^{*}$

Provision has been made in the program for fitting the betatron phase shift angle $\mu$, associated with the usual AGS treatment of magnet systems.

In the horizontal plane: use code digits $i=-11 ., j=2 .$, and specify:

$$
\begin{aligned}
\Delta=\frac{1}{2 \pi} \cos ^{-1}\left[0.5\left(R_{11}+R_{22}\right) \cdot\right] & =\frac{\mu}{2 \pi} \text { (horiz) } \\
& =\text { freq./(No. of periods) } .
\end{aligned}
$$

In the vertical plane: $i=-13 ., j=4 .$, and

$$
\Delta=\frac{1}{2 \pi} \cos ^{-1}\left[0.5\left(R_{33}+R_{44}\right)\right]=\frac{\mu}{2 \pi}(\text { ver } t) .
$$

For example, if there are 16 identical sectors to a proposed AGS machine and the betatron frequencies per revolution are to be 3.04 and 2.14 for the horizontal and vertical planes respectively, then the last element of the sector should be followed by the constraints:

$$
\begin{aligned}
& \text { 10. -11. 2. .190.001; } \\
& \text { 10. -13. 4. .134 .001; } \\
& \text { i.e. } \frac{3.04}{16}=0.190 \text { and } \frac{2.14}{16}=0.134 \text {. }
\end{aligned}
$$

For example: A typical data listing might be:

$$
\begin{array}{lll}
5.01 & - & \text {; } \\
3 . & -- & \text {; } \\
5.01 & -- & \text {; } \\
3 . & - & \text {; }
\end{array}
$$

10. -11. 2. 0.190 .001 ;

10. -13 . 4. 0.134 .001 ;

*) See Courant and Snyder ${ }^{1)}$. 


\section{T1 matrix fitting constraints}

Five parameters are needed for a constraint on the ( $i, j, k$ ) element of the second-order transfer matrix T1.

1 - Type code 10.0

2 - Code digit $(-i)$.

3 - Code digit $(10 j+k)$.

4 - Desired value of the $(i, j, k)$ matrix element.

5 - Desired accuracy of the fit (standard deviation).

Note that upper and lower limil cunstraints are not available for second order fitting.

Some typical T1 matrix constraints are as follows:

\begin{tabular}{|c|c|c|c|c|c|c|c|}
\hline Desired optical condition & \multicolumn{7}{|c|}{ Typical fitting constraint } \\
\hline Geometric aberration $\mathrm{T}_{122}=0$ & 10. & -1 & 22. & .0 & .001 & 'F1' & ; \\
\hline Chromatic aberration $T_{346}=.5$ & 10. & & 46. & .5 & .001 & 'F2' & ; \\
\hline
\end{tabular}

'lhere must be no updates of the $\mathrm{R} 2$ matrix when constraining an element of the Tl matrix. There is no limit on the number of constraints which may he imposed.

If no drift lengths are varied the problem will be linear and the absolute size of the tolerances will be unimportant. Only their relative magnitude will be significant. Sometimes only a subset of the elements of the matrix $\mathrm{T}_{i j k}$ which give significant contributions to beam dimensions need be eliminated. In such cases one may wish to minimize the effect of this subset, by weighing each matrix element according to its importance. One does this by including a constraint for each such matrix elemeut, and setting its tolerance equal to the inverse of the phase..space factor which the matrix element multiplies. For a matrix element $T_{i j k}$ acting on an uncorrelated initial phase space, the tolernnce factor would be $1 /\left(x_{0 j} x_{0_{k}}\right)$, where $x_{0 j}$ and $x_{0 k}$ are the initial beam half widths specified by the type code 1.0 card. 
T2 matrix fitting constraints

Five parameters are needed for a constraint on the (i, j, k) element of the second order auxiliary transfer matrix T2.

1 - Type code 10.0

2 - Code digit - $(20+i)$.

3 - Code digit $(10 j+k)$.

4 - Desired value of the (i, $j, k$ ) matrix element.

5 - Desired accuracy of the fit (standard deviation)

Note that upper and lower limit constraints are not available for secund-order fitting.

Some typical T2 matrix constraints are as follows:

\begin{tabular}{|c|c|c|c|c|c|c|c|}
\hline Desired optical condition & \multicolumn{7}{|c|}{ Typical fitting constraint } \\
\hline Geometric aberration $\mathrm{T}_{122}=0$ & 10 . & -21 & 22 . & .0 & .001 & 'Fl' & ; \\
\hline Chromatic aberration $\mathrm{T}_{346}=.5$ & 10 . & -23 . & 46. &. .5 & .001 & 'F2' & ; \\
\hline
\end{tabular}

By using a T2 constraint the user may fit an element of the secondorder transfer matrix which pertains to any section of the beam. One causes an R2 update at the beginning of the section with a 6.0 .2 . ; element. One then places the $\mathrm{T} 2$ constraint at the end of the section. Any number of such constraints may be imposed. This is the only secondorder constraint that may be used in conjunction with an R2 update.

If a printing of the $\mathrm{T} 1$ matrix is requested via a 13.4 ; element it will be the second-order transfer matrix from the last Rl update. The comments about phase space weighting, made in connection with the T1 constraint, are equally valid for the $\mathrm{T} 2$ constraint, provided the phase space factors are obtained from the beam matrix at the position of the R2 update. 


\section{Second-order $\sigma$ (BEAM) matrix fitting constraint}

Five parameters must be specified for a constraint on the secondorder contributions to a beam matrix diagonal element $\sigma_{i i}$.

1 - Type code 10.0

2 - Code digit (i).

3 - Code digit (i).

4 - The number 0 .

5 - Desired accuracy of the fit (standard deviation).

If, for example, one wished to minimize the net contributions of second-order aberrations to the horizontal divergence, one would insert the following card:

10. 2. 2. $\quad .0 \quad .01 \quad$;

The quantity that is minimized is the net increase due to secondorder terms in the second moment of the beam about the origin. This quantity is treated as the chi-squared of the problem, so the only meaningful desired value for the fit is zero. The square root of this quantity is printed in the output. It is computed using the $R_{2}$ matrix. Therefore, once again, one must not include any element which updates the $R_{2}$ matrix. Centroid shifts must not be inserted when doing second-order fitting, even immediately following the beam card.

The second-order image of the initial beam centruid at some later point in the beam is not necessarily the heam centroid at the latre point. The parameters printed by TRANSPORT are the new centrnid nosition and the beam matrix about the new centroid. One must therefore look at both of these to observe the effects of the fitting prncedure. Tt may evon happen that an improvement in one parameter will be accompanied by a slight deterioration in the other.

The beam profile at any point is a function of the initial beam parameters. One may therefore impose weights on the effect of the various aberrations by the choice of parameters on the BEAM card. One might, for example, adjust the strength of the correction of the chromatic aberrations wy lhe clivice of the $\Delta \mathrm{p} / \mathrm{p}$ parameter. In particular, when using a BEAM constraint, one should not attempt to minimize or eliminate chromatic aberrations if $\Delta p / p$ is set equal to zero on the beam card (type code 1.0 ).

Correlations (the 12.0 card) may also be included in the initial beam specification. 


\section{Sextupole strength constraints}

Five parameters must be specified for a constraint on sextupole strength.

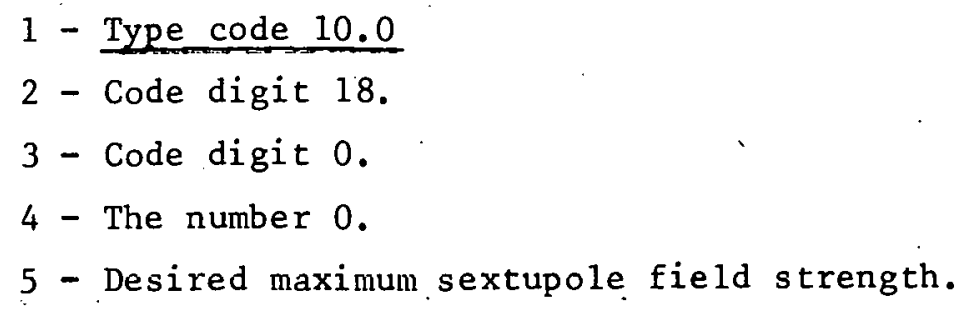

A single sextupole constraint card applies to all sextupoles which follow. The maximum field strength is treated as a standard deviation and may be exceeded on an optimal fit.

One can employ this constraint to find the optimal locations for sextupoles. By placing inversely coupled drift lengths before and after the sextupole its longitudinal position may be varied. By constraining the field strength the sextupole can be slid to a position where the coupling coefficients to the aberrations will be largest. One will need to experiment with adjusting the maximum field strength to achieve the best configuration. 
Internal constraints

A set of upper and lower bounds on the value of each type of parameter is in the memory of the program. If a correction is computed for a parameter which would take its value outside this range, it is reset to the limit of the range. The current limits are:

\begin{tabular}{l|c} 
Type code & Limits \\
\hline 1.0 & $0<$ input beam \\
2.0 & $-60<$ pole-face rotation $<60$ (deg) \\
3.0 & $0<$ drift \\
4.0 & $0<$ magnet length \\
5.0 & $0<$ quad length. \\
20.0 & $-360<$ beam rotation $<360$ (deg).
\end{tabular}

These limits apply only when a parameter is being varied. Fixed values that exceed this range may be used as desired.

These constraints were included to avoid physically meaningless solutions. 
Corrections and covariance matrix

When the program is fitting, it makes a series of runs through the beam line. From each run it calculates the chi-squared and the corrections to be made to the varied parameters. For each iteration a single line is printed containing these quantities.

The program calculates the corrections to be made using a matrix inversion procedure. However, because some problems are difficult, it proceeds with caution. The corrections actually made are sometimes a fixed fraction of those calculated. This fraction, used as a scaling factor, is the first item appearing on the line of printed output. The second factor is the chi-squared before the calculated corrections are made. Following are the corrections to be made to the varied parameters. They are in the order in which they appear in the beam line. If several parameters are coupled, they are considered as one and their position is determined by the first to appear.

When convergence has occurred, the final value of the chi-squared and the covariance matrix are printed. The covariance matrix is symmetric, so only a triangular matrix is shown. The diagonal elements give the change in each varied parameter needed to produce a unit increase in the chi-squared. The off-diagonal elements give the correlations between the varied parameters.

The appearance of the chi-squared and covariance matrix is: * COVARIANCE (FIT $\left.\chi^{2}\right)$

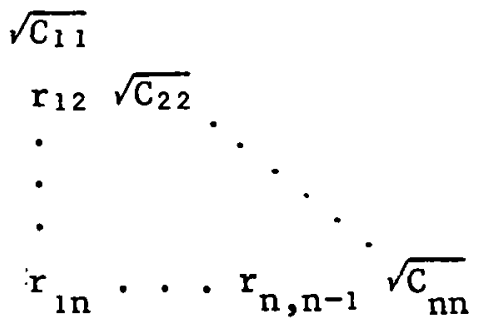

For more details on the mathematics of the fitting, the user should consult the Appendix. For an example of the output of the program he (or she) should refer to the section on output format. 


\section{ACCELERATION: Type code 11.0}

An energy gain is reflected in both the divergence and the width of the beam. This element provides a simulation of a travelling wave linear accelerator energy gain over a field free drift length (i.e. no externally applied magnetic field).

There are five parameters:

1 - Type code 11.0

2 - Accelerator length (metres).

3 - Energy gain (GeV).

4 - $\phi$ (phase lag in degrees).

$5-\lambda$ (wavelength in ciII).

The new beam energy is printed as nutput,

The energy of the reference trajectory is assumed to increase linear1y over the entire accelerator length. If this is not the case, an appropriate model may be constructed by combining separate 11.0 elements. An 11.0 element with a zero energy gain is identical to a drift length.

None of the parameters may be varied.

Second-order matrix'elements have not been incorporated in the program for the accelerator section.

The units of parameters 2,3 , and 5 are changed by $15,8,15.11 .$, and 15 . 5. type code entries respectively. 


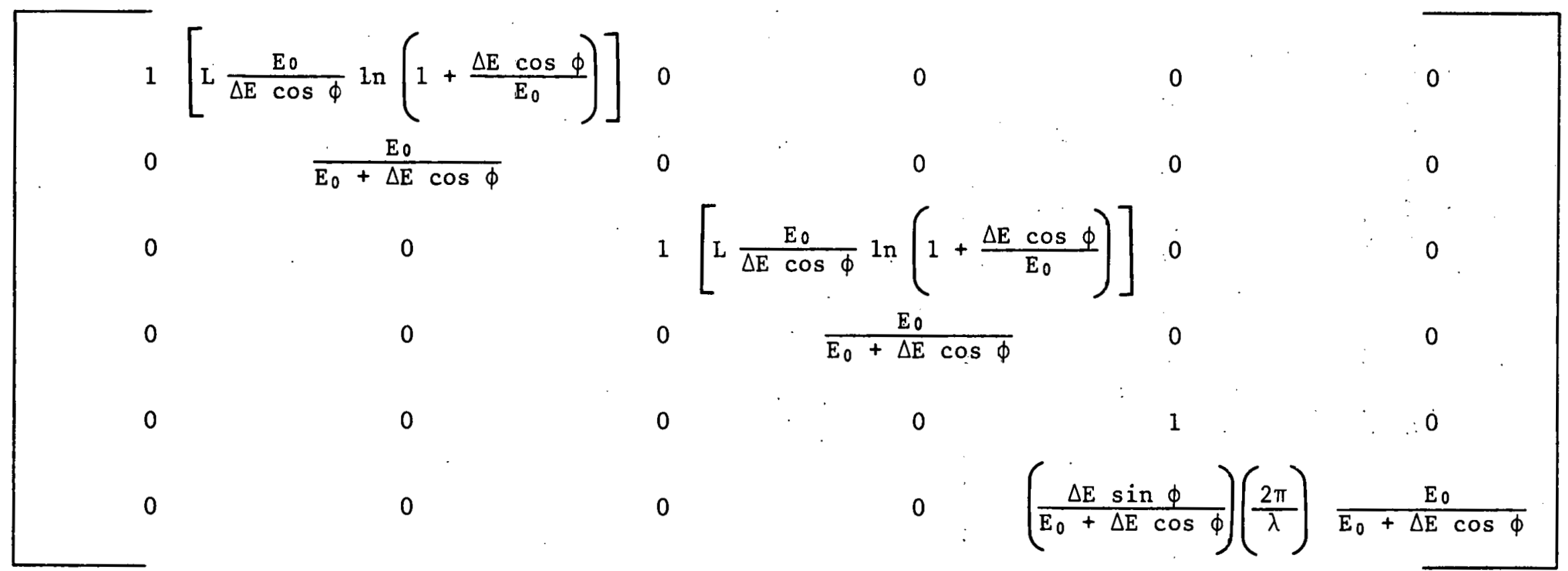

Definitions: $\quad L=$ effective length of accelerator sector

$$
E_{0}=\text { particle energy at start of sector }
$$

$\Delta \mathrm{E}=$ energy gain over sector length

$\phi=$ phase $1 \mathrm{ag}$ of the reference particle behind the crest of the accelerating wave, i.e. if $\phi$ is positive then for some $\ell>0$ the particles having this value are riding the crest of the wave; the units of $\phi$ are degrees

$\lambda=$ wavelength of accelerating wave; the units of $\lambda$ are those of $\ell$ (normally $\mathrm{cm}$ ).

This matrix element assumes that $E_{0} \gg m_{0} c^{2}$ (fully relativistic). 


\section{BEAM (rotated ellipse): Type code 12.0}

To allow the output beam from some point in a system to become the input beam of some succeeding system, provision has been made for reentering the correlation matrix which appears as a triangular matrix in the beam output. (See section under type code 1.0 and/or the Appendix for definitions.)

There are 16 parameters:

$$
1 \text { - Type code } 12.0
$$

2 to 16 - The 15 correlations $(r(i j)$ ) among the 6 beam components in the order printed (by rows).

Several cards may be used to insert the 15 correlations, if necessary.

Since this element is solely an extension of the beam input, a 12.0 element must immediately be preceded by a 1.0 (BEAM) element entry.

The effect of this element in the printed output is shown only in the beam matrix. If the beam matrix is printed automatically, it is not printed directly after the BEAM element but only after the correlation matrix has been inserted. 
Output PRINT CONTROL instructions: Type code 13.0

A number of control codes which transmit output print instructions to the program have been consolidated into a single type code:

There are two parameters:

1 - Type code 13.0

2 - Code number.

The effects of the various code numbers will be described below (not in numerical order).

Printed output controls 1.2,2.,23., 4.,24.

Several codes are available to control various aspects of the printed output. Most type codes produce a line of output that advertises their existence. Those that do not, usually have an obvious effect upon the remainder of the output and thus make their presence clear.

(13. 2. ;): This code instructs the program to suppress the printing of the beam $(\sigma)$ matrix.

(13. 1. ;): This code temporarily overrides the (13. 2. ;) suppress code. It causes a single beam matrix to be printed at that point in the sys tem.

(13. 3. ;): This code permanently overrides the (13. 2. ;) suppress code and restores the normal code in which every beam matrix is printed.

(13. 4. ;): This code instructs the program to print the current transformation matrix R1 (TRANSFORM 1). If the program is computing a second-order matrix, this second-order transformation matrix will be included in the print-out. This matrix is cumulative from the last RI (TRANSFORM 1) update. The units of the elements of the printed matrix are consistent with the input units associated with the type code 1.0 (BEAM) entry.

(13. 24. ;): This code causes the TRANSFORM 2 matrix, R2, to be printed. The format and units of R2 are identical with those of R1 which is printed by the (13.4. ;) code. For a list of elements which update the R2 matrix, see type code 6 . 
The units of the tabulated matrix elements in either the firstorder $R$ or sigma matrix or second-order $T$ matrix of a TRANSPORT printout will correspond to the units chosen for the BEAM card. For example, the $R(12)=(x / \theta)$ matrix element will normally have the dimensions of $\mathrm{cm} / \mathrm{mr}$; and the $\mathrm{T}(236)=(\theta / \mathrm{y} \delta)$ matrix element will have the dimensions $\mathrm{mr} /(\mathrm{cm}-$ percent $) \Delta \mathrm{p} / \mathrm{p}$ and so forth.

\section{Coordinate layout control 12}

One can produce a layout of a beam line in any Cartesian coordinate system one chooses. The coordinates printed represent the $x, y$, and $z$ position, respectively, of the reference trajectory at the interface between two elements. In the printed output the values given are those at the exit of the element listed above and at the entrance of the element 1 isted immediately below.

A layout is specified by placing a (13. 12. ;) card before the beam card. If no additional cards are inserted the reference trajectory of the beam line will be assumed to start at the origin and proceed along the positive z-axis. The y-axis will point up and the $x$-axis to the left. One can also specify other starting coordinates and orientations by placing certain other cards before the beam card. For a description of such cards see type code 16.0 (special parameters).

The three cocrdinates and the accumulated length along the reference trajectory are printed to the left of the beam matrix. They appear whenever the beam matrix appears. The user should read in this section under printed output controls for a description of the elements which control the printing of the heam matrix,

The calculation of the coordinates is done from the parameters of the physical elements as given in the data. Therefore, if effective lengths are given for magnetic elements, the coordinates printed will be thuse at the effective field boundary. 'lhe effects of fringing fields in bending magnets are not taken into account.

Punched output controls 29, 30, 31, 32, 33, 34, 35, 36

If the control is equal to 29 , all of the terms in the first-order matrix and the $x$ and $y$ terms of the second-order matrix are punched. 
If the control is equal to 30 , all of the terms of the first-order matrix and all second-order matrix elements are punched out.

If the control, $\mathrm{n}$, is greater than 30 , all of the first-order terms are punched and the second-order matrix elements which correspond to $(n-30$.$) i.e., if n=32$, the second-order theta matrix elements are punched out. If $\mathrm{n}=31$, the second order $\mathrm{x}$ matrix elements are punched and so forth. 


\section{ARBITRARY TRANSFORMATION input: Type code 14.0}

To allow for the use of empirically determined fringing fields and other specific (perhaps non-phase-space-conserving) transformations, provision has been made for reading in an arbitrary transformation matrix. The first-order $6 \times 6$ matrix is read in row by row.

There are eight parameters for each row of a first-order matrix entry:

1 - Type code 14.0

2 to 7 - The six numbers comprising the row. The units must be those used to print the transfer matrix; in other words, consistent with the BEAM input/output.

8 - Row number (1, to 6.)

A complete matrix must be read and applied one row at a time. Rows that do not differ from the unit transformation need not be read.

For example, (14. -.1 .9 0.0.0.0.2. ;) introduces a transformation matrix whose second row is given but which is otherwise a unit matrix. Note that this transformation does not conserve phase space because $R(22)=0.9$, i.e. the determinant of $R \neq 1$.

Any of the components of a row may be varied; however, there are several restrictions.

Type code 14.0 ements that immediately follow one another will all be used to form a oingle transformation matrix. If distinct matrices are desired, another element must be inserted to separate the type code 14.0 cards. Several do-nothing elements are available; for example, a zero length drift (3.0. ;) is a convenient one.

When the last of a sequence of type code 14.0 cards is read, the assembled transformation matrix will be printed in the output. 
Note that

$$
\left(\begin{array}{ll}
1 & 0 \\
a_{21} & a_{22}
\end{array}\right)\left(\begin{array}{ll}
a_{12} & a_{12} \\
0 & 1
\end{array}\right) \neq\left(\begin{array}{ll}
a_{11} & a_{12} \\
a_{21} & a_{22}
\end{array}\right)
$$

Hence, a matrix formed by successive 14 . (3. 0 . ;), 14 - elements is not always equal to the one formed by leaving out the $(3.0 . ;)$ element.

If components of a 14.0 card are to be varied it must be the last 14.0 card in its matrix. This will force a matrix to be split into factors if more than one row has variable components.

If it is desired to read in the second-order matrix coefficients for the $i^{\text {th }}$ row, then the following 22 additional numbers may be read in ${ }^{* *}$.

9 - continuation code 0 .

10 to 30 - the 21 coefficients:

\begin{tabular}{|c|c|c|c|c|c|}
\hline $\mathrm{T}(\ddot{i} 11)$ & $\mathrm{T}(\mathrm{i} 12)$ & $\mathrm{T}$ (i13) & $\mathrm{T}(\mathrm{i} 14)$ & $\mathrm{T}(\mathrm{i} 15)$ & $\mathrm{T}(\mathrm{i} 16)$ \\
\hline $\mathrm{T}$ (i22) & $\mathrm{T}(\mathrm{i} 23)$ & $T($ i24) & $\mathrm{T}(\mathrm{i} 25)$ & $T(i 26)$ & $\mathrm{T}(\mathrm{i} 33)$ \\
\hline $\mathrm{T}(\mathrm{i} 34)$ & $T(i 35)$ & $T(i 36)$ & $\mathrm{T}(\mathrm{i} 44)$ & $T(i 45)$ & $T(i$ \\
\hline 1 & (i56) & $\mathrm{T}(\mathrm{i} 66)$ & & & \\
\hline
\end{tabular}

in that order, where $i$ is the row number. It is necessary to read in the first-order matrix row which corresponds to the second-order matrix row being read in.

As in the first-order case, full rows not different from the identity matrix [i.e., $R(i i)=1$, all other $R(i j)=0$, and all $T(i j k)=0]$ need not be read. in.

\footnotetext{
**) This feature frees the user from making repetitive, expensive, second-order runs through a fixed portion of his system while experimenting with other magnets. This is done by reading the full matrix of this portion (obtained from a previous run) back into the machine as a single "arbitrary matrix."
} 
Input-output UNITS: Type code 15.0

TRANSPORT is designed with a standard set of units that have been used throughout this manual. However, to accommodate other units conveniently, provision has been macie for redefining the units to be employed. This is accomplished by insertion of one or more of the following elements.

There are four parameters to be specified:

1 - Type code 15.0

2 - Code digit.

3 - The abbreviation of the unit (see examples below). This will be printed on the output listing. It must be enclosed in eingle quotes and is a meximum of three characters long (four for energy). The format for insertion is the same as for labels.

4 - The scale factor (if needed). The scale factor is the size of the new unit relative to the standard TRANSPORT unit. For example, if the new unit is inches and the standard TRANSPORT unit $\mathrm{cm}$, the scale factor is (2.54). 
The various units that may be changed are:

\begin{tabular}{|c|c|c|c|}
\hline $\begin{array}{l}\text { Code } \\
\text { Digit }\end{array}$ & Quantity & $\begin{array}{l}\text { Standard } \\
\text { TRANSPORT Unit }\end{array}$ & $\begin{array}{c}\text { Symbols used in } \\
\text { SLAC-75 }\end{array}$ \\
\hline 1.0 & $\begin{array}{l}\text { horizontal and vertical } \\
\text { transverse dimensions, } \\
\text { magnet apertures and mis- } \\
\text { alignment displacements. }\end{array}$ & $\mathrm{cm}$ & $\mathrm{x}, \mathrm{y}$ \\
\hline 2.0 & $\begin{array}{l}\text { horizontal and vertical } \\
\text { angles and misalignment } \\
\text { rotation angles }\end{array}$ & $\mathrm{mr}$ & $\theta, \phi$ \\
\hline 3.0 & $\begin{array}{l}\text { vertical beam extent } \\
\text { (only) }{ }^{*} \text { and hending } \\
\text { magnet gap height }\end{array}$ & $\mathrm{cm}$ & $\mathrm{y}$ \\
\hline 4.0 & $\begin{array}{l}\left.\text { vertical beam divergence }{ }^{*}\right) \\
\text { (on } 1 y)\end{array}$ & $\mathrm{mr}$ & $\phi$ \\
\hline 5.0 & $\begin{array}{l}\text { pulsed beam length and } \\
\text { wave length in accelerator }\end{array}$ & $\mathrm{cm}$ & $\ell$ \\
\hline 6.0 & momentum spread & percent (PC) & $\delta$ \\
\hline 7.0 & undefined & & \\
\hline 8.0 & length (1ongitudinal) & metres (M) & $t$ \\
\hline & $\begin{array}{l}\text { of elements, layout co- } \\
\text { ordinated and bending } \\
\text { magnet pole face curvatures }\end{array}$ & & . \\
\hline 9.0 & magnetic fields & $\mathrm{kG}$ & B \\
\hline 10.0 & mass & electron mass & $\mathrm{m}$ \\
\hline 11.0 & momentum and & $\mathrm{GeV} / \mathrm{c}$ & $p(0)$ \\
\hline & $\begin{array}{l}\text { energy gain in accelerator } \\
\text { section }\end{array}$ & $\mathrm{GeV}$ & $\Delta E$ \\
\hline
\end{tabular}

*) These codes should not be used if the coordinate rotation (20.0) type code is used anywhere in the system. 
Units are not normally restored at the end of a problem step. Once changed, they remain the same for a11. succeeding problem steps in an input deck until a 0 indicator card is encountered, at which time they are reset to standard TRANSPORT units. The units may be reset to standard units by inserting a (15. ;) type code entry.

The 15.0 elements are the first cards in a deck (immediately following the title card and the 0 or 1 indicator card) and should not be inserted in any other location. They produce no printed output during the calculation, their effect being visible only in the output from other elemento.

Example: To change leuglh to feer, width to inches, and momentum to $\mathrm{MeV} / \mathrm{c}$, add to the front of the deck the elements

$$
\begin{array}{rrrl}
\text { 15. } & \text { 8. ' } \mathrm{FT} & 0.3048 \text {; } \\
\text { 15. 1. ' } \mathrm{IN} \text { ' } 2.54 \text {; } \\
\text { 15. 11. 'MEV' } 0.001 \text {; }
\end{array}
$$

The scale factor, 0.3048 , multiplies a length expressed in the new unit, feet, to convert it to the reference unit, metres, etc.

For the conventional units listed below, it is sufficient to stop with the label (the conversion factor is automatically inserted by the program). If units other than those listed below are desired, then the label and the appropriate conversion factor wust be included. If the automatic feature is used with older versions of the program, there must be no blank spaces between the quotes and the labels. 
Input-output units: Type code 15.0

(Conversion factors for dimension changes versus code digit and label)

\begin{tabular}{|c|c|c|c|c|c|c|c|c|c|c|c|}
\hline LABEL & \multicolumn{11}{|c|}{ CODE DIGIT } \\
\hline & 1. & 2. & 3. & 4. & 5. & 6. & 7. & 8. & 9. & 10. & 11. \\
\hline 'CM' & 1. & --- & 1. & --- & 1. & --- & --- & .01 & -- & -- & --- \\
\hline 'M' & 100. & -- & 100. & --- & 100. & --- & --- & 1. & --- & -- & --- \\
\hline 'IN' & 2.54 & --- & 2.54 & --- & 2.54 & --- & $--\dot{x}$ & .0254 & --- & -- & $-\cdots$ \\
\hline 'FT' & 30.48 & --- & 30.48 & --- & 30.48 & --- & -- & .3048 & -- & -- & --- \\
\hline 'MM' & .1 & --- & .1 & --- & .1 & --- & --- & .001 & -- & $\cdots$ & -- \\
\hline 'R' & -- & 1000. & -- & 1000. & --- & --- & --- & -- & -- & --- & -- \\
\hline 'MR' & -- & 1. & --- & 1. & -- & --- & --- & -- & -- & -- & -- \\
\hline 'PC' & -- & --- & -- & -- & -- & 1. & -- & -- & --- & -- & --- \\
\hline $\mathrm{P} / 10^{\circ}$ & -- & --- & -- & --- & --- & .1 & --- & -- & -- & --- & -- \\
\hline 'N' & -- & -- & -- & --- & --- & 100. & -- & -- & -- & -- & --- \\
\hline 'MEV' & --- & --- & -- & --- & -- & -- & -- & $-\cdots$ & -- & --- & .001 \\
\hline 'GEV' & --- & $\cdots$ & -- & --- & --- & -- & --- & --- & -- & --- & 1. \\
\hline 'KG' & --- & --- & --- & --- & --- & -- & -- & --- & 1. & --- & -- \\
\hline 'G' & --- & --- & --- & --- & --- & -- & --- & --- & .001 & -- & $\because-$ \\
\hline
\end{tabular}

$P C$ is an abbreviation for percent $P / 10$ means one-tenth of a percent $\mathrm{N}$ means 100 percent 
SPECIAL INPUT PARAMETERS: Type code 16.0

A number of constants are used by the program which do not appear as parameters in elements of any other type code. A special element has been provided to allow the designer to set their values. These special parameter entries must always precede the physical element(s) to which they apply. Once introduced, they apply to all succeeding elements in the beam line unless reset to zero or to new values.

There are three parameters:

1 - Type code 16.0

2 - Code digit.

3 - Value of the constant.

A number of such constants have been defined in this manner. All have a normal value that is initialized at the beginning of each run. Code digits for special parameters

1. $\varepsilon(1)$ - a second-order measure of magnetic field inhomogeneity in bending magnets. If

$B(x)=B(0)\left[1-n\left(\frac{x}{\rho_{0}}\right)+B\left(\frac{x}{\rho_{0}}\right)^{2}-\ldots\right]$

is the field expansion in the median ( $y=0)$ plane, then

$\varepsilon(1)$ is defined as

$\varepsilon(1)=B\left(\frac{1}{\rho_{0}}\right)$

(where $\rho_{0}$ is measured in units nf horizontal beam width normally $\mathrm{cm}$ ). This parameter affects second-order calculations unly. Normally the value it 0 . It may be varied in second-order fitting.

3. $(M / m)$ - Mass of the particles comprising the beam, in units of the electron mass; normally 0. A non-zero mass introduces the dependence of pulse length on velocity, an important effect in low-energy pulsed beams.

4. W/2 - Horizontal half-aperture of hending magnet, in the came units as horizontal beam width, normally 0 (i.e. effect of hurizontal half aperture is ignored). 
5. $g / 2$ - Vertical half-aperture of bending magnet, in the same units as vertical beam height; this parameter must be inserted if the effect of the spatial extent of the fringing fields upon transverse focusing is to be taken into account. (See type codes 2.0 and 4.0 as a cross reference) normally 0.

6. L - Cumulative length of system, in the same units as system length. It is set to zero initially, then increased by the length of each element, and finally printed at the end of the system. This element allows the cumulative length to be reset as desired.

7. $K_{1}$ - An integral related to the extent of the fringing field of a bending magnet. See section under type code 2.0 and. SLAC-75 page 74 for further explanation. If the (16. 5. g/2. ;) element has been inserted, the program inserts.. default value of $\mathrm{K}_{1}=\frac{1}{2}$ unless a $\left(16,7 . \mathrm{K}_{1}\right.$. ;) element is introduced, in which case the program uses the $K_{I}$ value selected by the user. The table below shows typical values for various types of magnet designs.

8. $\mathrm{K}_{2}$. - A second integral related to the extent of the fringing field. Default value of $\mathrm{K}_{2}=0$ unless specified by a (16. 8. $\mathrm{K}_{2}$. ;) entry.

Typical values of $K_{1}$ and $K_{2}$ are given below for four types of fringing field boundaries:
a) a linear drop-off of the field,
b) a clamped "Rogowski" fringing field,
c) an unclamped "Rogowski" fringing field,
d) a "square-edged" non-saturating magnet 


\begin{tabular}{|c|c|c|}
\hline Model & $\mathrm{K}_{1}$ & $\left.\mathrm{~K}_{2}{ }^{*}\right)$ \\
\hline Linear & $1 / 6$ & 3.8 \\
Clamped Rogowski & 0.4 & 4.4 \\
Unclamped Rogowski & 0.7 & 4.4 \\
Square-edged magnet & 0.45 & 2.8 \\
\hline
\end{tabular}

12. $1 / R_{1}$ - Where $R_{1}$ is the radius of curvature (in units of longitudinal length, normally metres) of the entrance face of bending magnets. (See figure on $\mathrm{p}$.

13. $1 / R_{2}$ - Where $R_{2}$ is the radius of curvature (in units of longitudinal length, normally metres) of the exit face of bending magnets. (See figure on $p$.

The pole face curvatures $\left(1 / R_{1}\right)$ and $\left(1 / R_{2}\right)$ affect the system only in second-order, creating an effective sextupole component in the neighbourhond of the magnet. If the parameters are not specified, they are assumed to be zero, i.e. no curvature and hence no sextupole component. Either parameter (or both) may be varied in second-order fitting.

*) For most applications $\mathrm{K}_{2}$ is unimportant. If you find it is important to your result you should probably be making a more accurate calculation with a ray-tracing program (see References at the end of the manual.) 


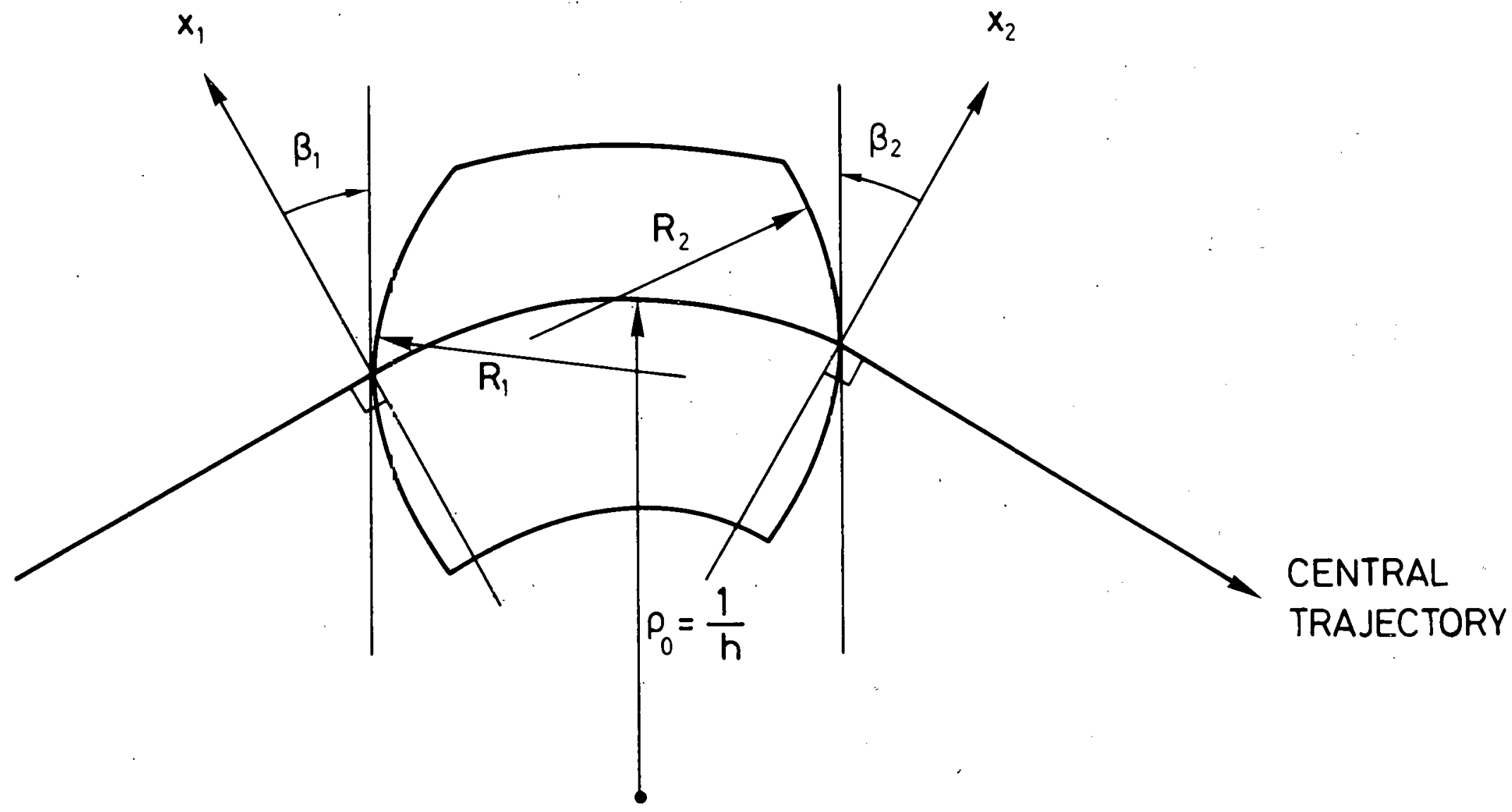

FIELD BOUNDARIES FOR BENDING MAGNETS

The TRANSPORT sign conventions for $x, \beta, R$ and $h$ are all positive as shown in the figure. The positive y direction is out of the paper. Positive $\beta^{\prime}$ s imply transverse focusing. Positive $R^{\prime}$ s (convex curvatures) represent negative sextupole components of strength $\mathrm{S}=(-\mathrm{h} / 2 \mathrm{R}) \sec ^{3}$ B. (See SLAC-75, page 71.) 
Tilt-to-focal plane (16. 15. a. ;) element

Very often it is desired to have a listing of the second-order aberrations along the focal plane of a system rather than perpendicular to the optic axis, i.e. along the $x$ coordinate. If the focal plane makes an angle $\alpha$ with respect to the $\mathrm{x}$ axis (measured clockwise) then provision has been made to rotate to this focal plane and print ont the second-order aberrations. This is achieved by the following procedures:

Alpha is the focal-plane tilt angle (in degrees) measured from the perpendicular to the optic axis ( $\alpha$ is normally zero).

The programming procedure for a tilt in the $x$ (bend)-plane (rotation about $\mathrm{y}$ axis) is:

$$
\begin{aligned}
& \text { 16. 15. a. ; } \\
& \text { 3. } 0 . \text {; (a necessary do-nothing element) } \\
& \text { 13. 4. ; } \\
& \text { 16. 15. }-\alpha \text {; (rotate back to zero) } \\
& \text { 3. } 0 . \text {; (a necessary do-nothing element) } \\
& \text { 16. 15. 0. ; (to turn off rotation element) }
\end{aligned}
$$


The programming procedure for a tilt in the $y$-plane (rotation about $\mathrm{x}$-axis) is :

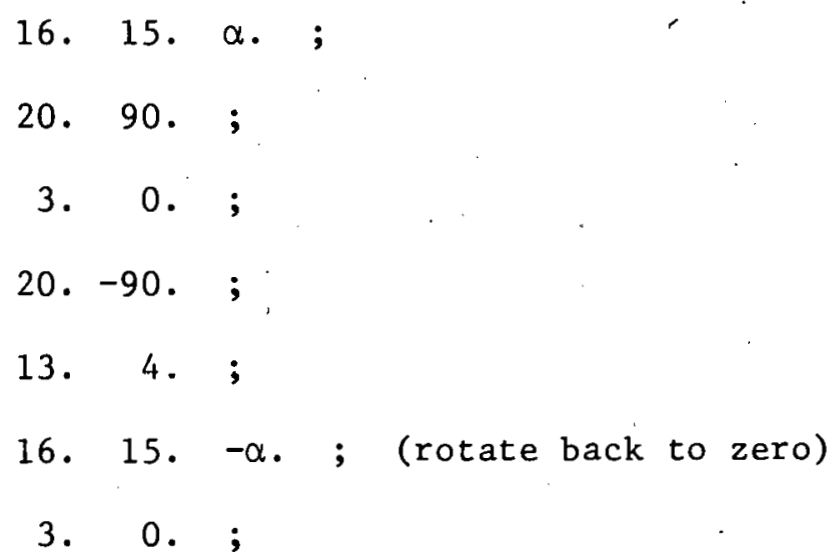

16. 15. 0 ; ; (to turn off rotation element) 


\section{Initial beam line coordinates and direction}

When requesting a beam line coordinate layout via a (13. 12, ;) element one can employ any coordinate system one desires. The position and direction of the beginning of the reference trajectory in this coordinate system are given on elements 16. 16. through 16.20. Such cards should be placed before the beam card, but after any units changes. Their meanings are as follows:

16.
16.
16.
16. $\quad \begin{aligned} & \mathrm{x}_{0}, \mathrm{y}_{0}, \text { and } \mathrm{z}_{0}, \text { respectively, the coordinates of } \\ & \text { the initial point of the reference trajectory } \\ & \text { in the units chosen for longitudinal length, } \\ & \text { 16. }\end{aligned}$

When specifying the initial orientation of the reference trajectory via the two angles, one must give the horizontal angle first. The meaning of the two angles is given in the following figure. Any of the above five parameters not explicitly specified will be taken to equal zero. 


\section{REFERENCE}

\section{TRAJECTORY}

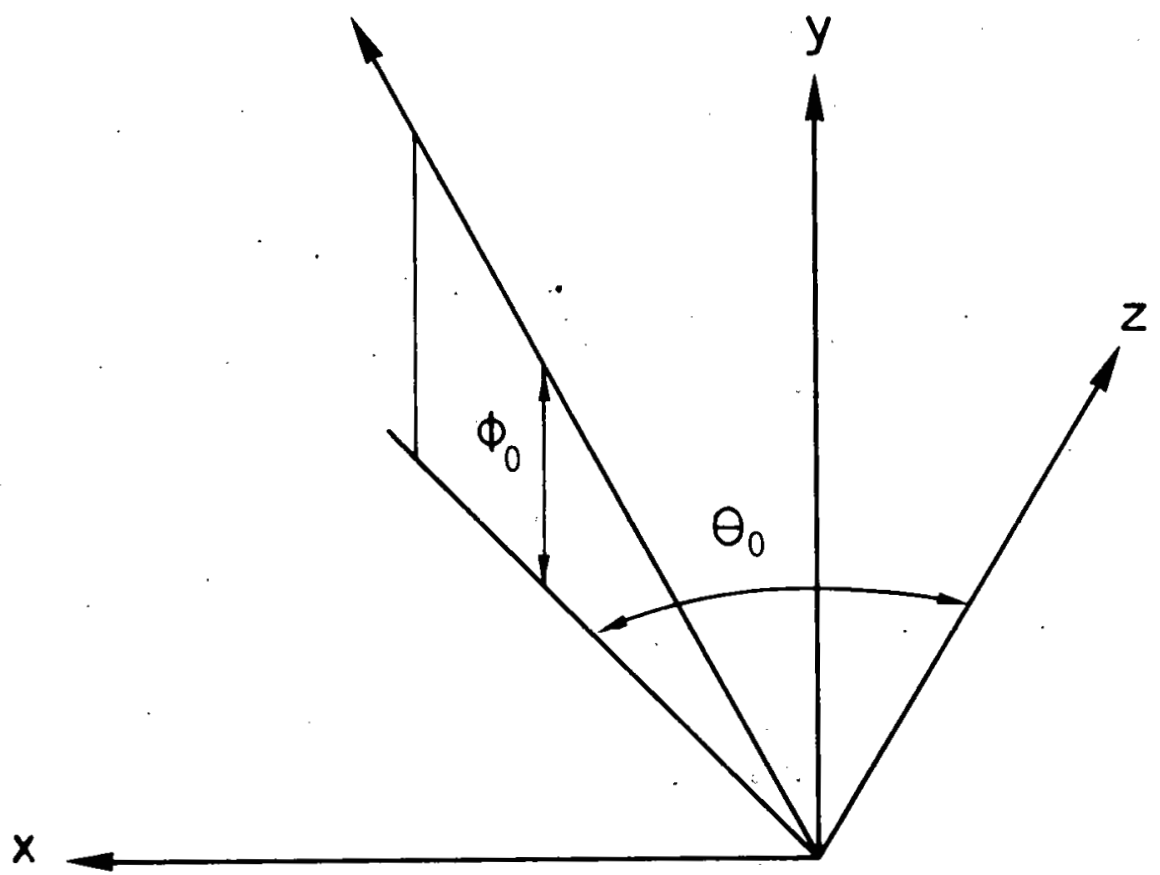

SPECIFICATION OF INITIAL ANGLES $\theta_{0}$ AND $\phi_{0}$ FOR BEAM LINE LAYOUT. 
SECOND-ORDER CALCULATION: Type code 17.0

A second-order calculation may be obtained provided no alignments are employed. A special element instructs the program to calculate the secondorder matrix elements. It must be inserted immediately following the beam (1. element).

Only one parameter should be specified:

1 - Type code 17.0 (signifying a second-order calculation is to be made).

To print out the second order T1 matrix terms at a given location in the system, the (13. 4. ;) print control card is used. For T2, the (13. 24. ;) print control card is used. The update rules are the same as those for the corresponding first-order $\mathrm{R}$ matrix. See SLAC-75 for definitions of subscripts in the second order $T(i j k)$ matrix elements.

The values of the BEAM (sigma) matrix components may he perturhed from their first-order value by the second-order aberrations. In a second-order TRANSPORT calculation, the initial beam is assumed to have a Gaussian distribution. For exact details the reader should consult the Appendix. For the beam matrix to be calculated correctly, there should be no elements which update the $\mathrm{R} 2$ matrix. If a centroid shift is present, it must immediately follow the beam (type code 1.0) or beam rotated ellipse (type code 12.0 ) card.

Only second-order fitting may be done in a second-order run. See the section on type code 10.0 for a list of quantities that may be constrained in a second-order run. If a beam constraint is to be imposed in second-order, there must be no centroid shifts present anywhere.

Second-order matrices are included in the program for quadrupoles, bending magnets (including fringing fields), the arbitrary matrix, sextupoles, and solenolds. They have not been calculated for the acceleration (type code 11.0) element. 
SEXTUPOLE: TYPe code 18.0

Sextupole (hexapole) magnets are used to modify second-order aberrations in beam transport systems. The action of a sextupole on beam particles is a second and higher order effect, so in first order runs (absence of the 17.0 card) this element will act as a drift space.

There are four parameters:

1 - Type code 18.0

2 - Effective length (metres).

3 - Field at pole tips (kG). Both positive and negative fields are possible (see figures below).

4 - Half-aperture $(\mathrm{cm})$. Radius of circle tangent to pole tips.

Other orientations of the sextupole may be obtained using the beam rotation element (type code 20.0 ).

The pole tip field may be varied in second-order fitting. It may also be constrained not to exceed a certain specified maximum field. (See the explanation of vary codes in the section on type code 10.0). Such a constraint allows one to take into account the physical realities of limitations on pole tip fields.

See SLAC-75 for a tabulation of sextupole matrix elements. The TRANSPORT input format for a typical data set is:

18. L. b. a. $\sqrt{\text { Label if desired (not to exceed }} \begin{gathered}4 \text { spaces) } \\ \text { (n) }\end{gathered}$ 


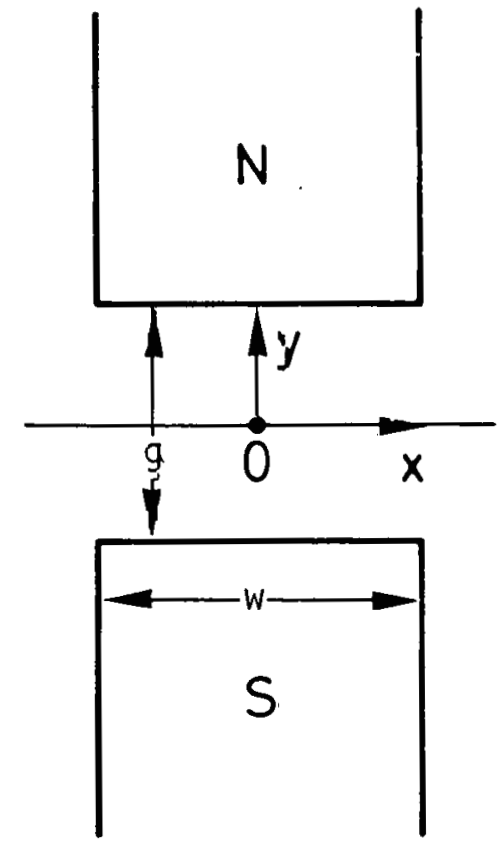

DIPOLE

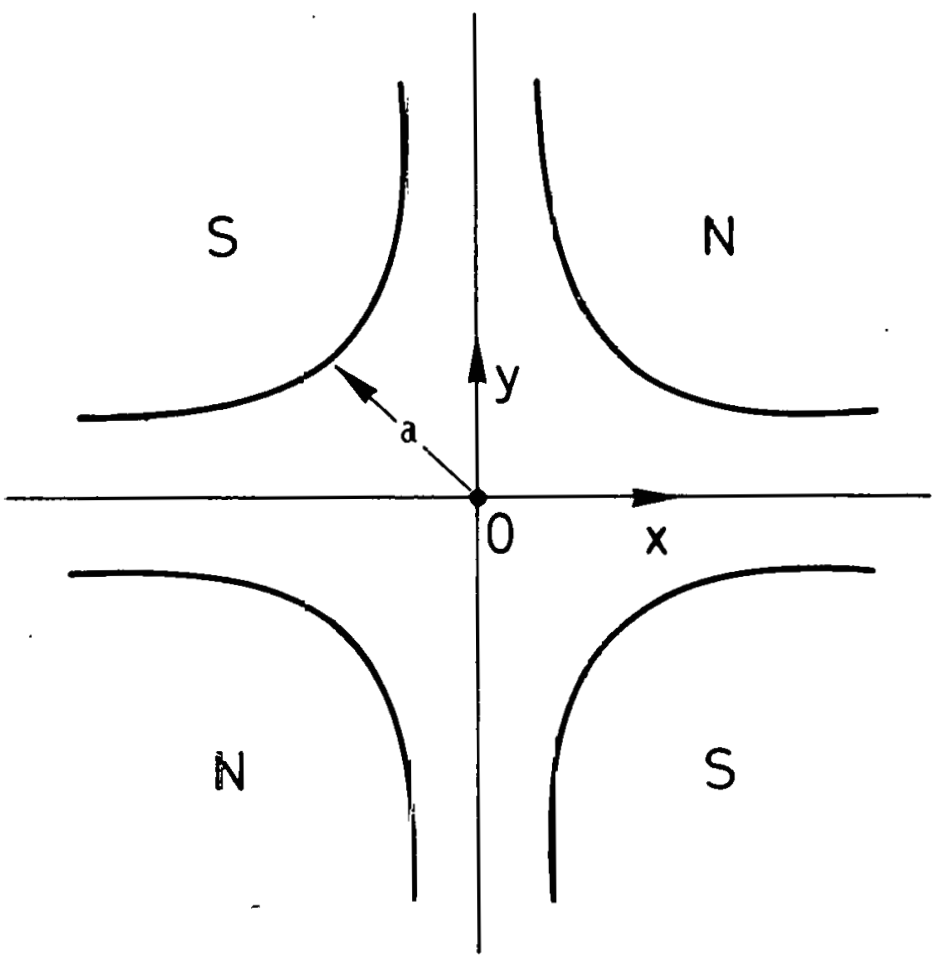

QUADRUPOLE

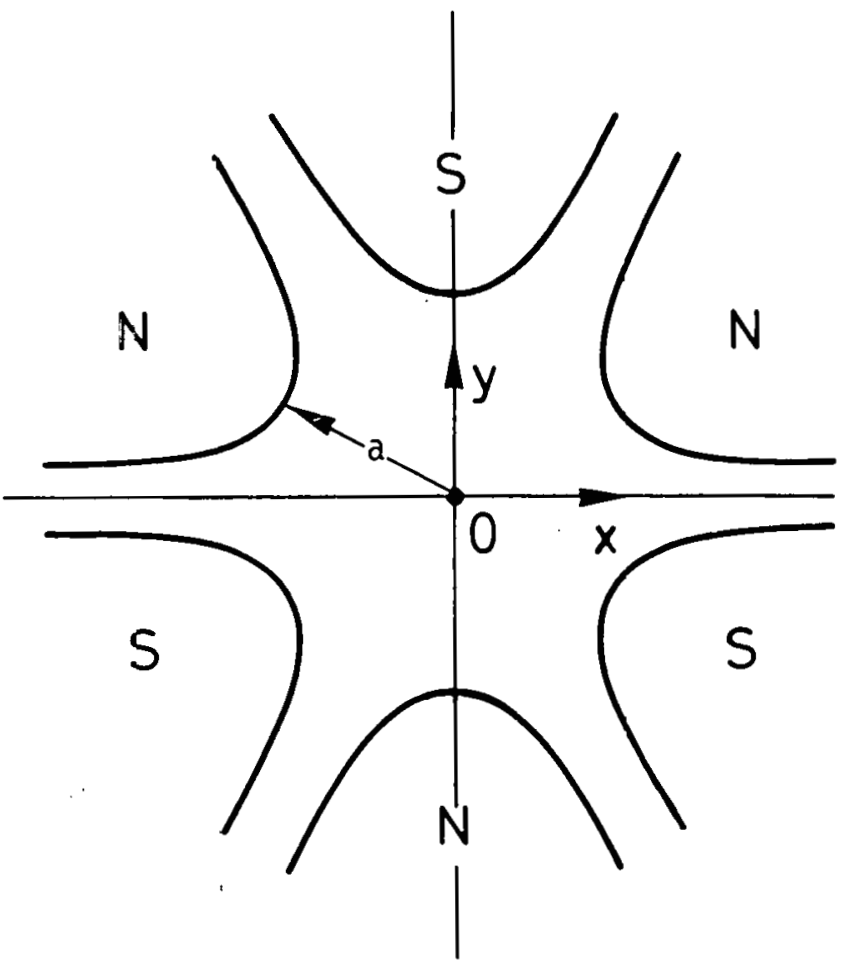

SEXTUPOLE

ILLUSTRATION OF THE MAGNETIC MIJPLANE ( $\mathrm{x}$ AXIS) FOR DIPOLE, QUADRUPOLE AND SEXTUPOLE ELEMENTS. THE MAGNET POLARITIES INDIZATE YULTIPDLE ELEMENTS THAT ARE POSITIVE WITH FESPECT TO EACH OTHER. 
SOLENOID: Type code 19.0

The solenoid is most often used as a focusing element in systems passing low-energy particles. Particles in a solenoidal field travel along helical trajectories. The solenoid fringing field effects necessary to produce the focusing are included.

There are three parameters:

1 - Type code 19.0

2 - Effective length of the solenoid (metres).

3 - The field (kG). A positive field, by convention; points in the direction of positive $z$ for positively charged particles.

The length and the field may be varied in first-order fitting. Both first- and second-order matrix calculations are available for the solenoid.

A typical input format is:

19. L. B. ' ' ; 


\section{First-order solenoid matrix}

Solenoid $\mathrm{R}$ matrix

Definitions: $L=$ effective length of solenoid

$$
\begin{aligned}
\mathrm{K}= & \mathrm{B}(0) /\left(2 \mathrm{~B} \rho_{0}\right), \text { where } \mathrm{B}(0) \text { is the field inside the } \\
& \text { solenoid and }\left(\mathrm{B} \rho_{0}\right) \text { is the (momentum) of the central } \\
& \text { trajectory. } \\
\mathrm{C}= & \cos \mathrm{KL} \\
\mathrm{S}= & \operatorname{cin} \mathrm{KL}
\end{aligned}
$$

For a derivation of this tranformation see report SLAC-4 by R. Helin.

Alternate forms of matrix representation of the solenoid:

$\mathrm{R}($ Solenoid $)=\left[\begin{array}{c|c|c|c|c|c}\mathrm{C}^{2} & \frac{1}{\mathrm{~K}} \mathrm{SC} & \mathrm{SC} & \frac{1}{\mathrm{~K}} \mathrm{~S}^{2} & 0 & 0 \\ -\mathrm{KSC} & \mathrm{C}^{2} & -\mathrm{KS}^{2} & \mathrm{SC} & 0 & 0 \\ -\mathrm{SC} & -\frac{1}{\mathrm{~K}} \mathrm{~S}^{2} & \mathrm{C}^{2} & \frac{1}{\mathrm{~K}} \mathrm{SC} & 0 & 0 \\ \mathrm{KS}^{2} & -\mathrm{SC} & -\mathrm{KSC} & \mathrm{C}^{2} & 0 & 0 \\ 0 & 0 & 0 & 0 & 1 & 0 \\ 0 & 0 & 0 & 0 & 0 & 1\end{array}\right]$


Rotating the transverse coordinates about the $z$ axis by an angle, $=-\mathrm{KL}$ decouples the $\mathrm{x}$ and $\mathrm{y}$ first-order terms, i.e.

$$
\mathrm{R}(-\mathrm{KL}) \cdot \mathrm{R} \text { (Solenoid) }=\left(\begin{array}{c|c|c|c|c|c}
\mathrm{C} & \frac{\mathrm{l}}{\mathrm{K}} \mathrm{S} & 0 & 0 & 0 & 0 \\
-\mathrm{KS} & \mathrm{C} & 0 & 0 & 0 & 0 \\
0 & 0 & \mathrm{C} & \frac{1}{\mathrm{~K}} \mathrm{~S} & 0 & 0 \\
0 & 0 & -\mathrm{KS} & \mathrm{C} & 0 & 0 \\
0 & 0 & 0 & 0 & 1 & 0 \\
0 & 0 & 0 & 0 & 0 & 1
\end{array}\right)
$$


COORDINATE ROTATION: Type code 20.0

The transverse coordinates $\mathrm{x}$ and $\mathrm{y}$ may be rotated through an angle $\alpha$ about the $z$ axis (the axis tangent to the central trajectory at the point in question) ${ }^{* *}$ ). Thus a rotated bending magnet, quadrupole, or sextupole may be inserted into a beam transport system by preceding and following the element with the appropriate coordinate rotation. (See examples below.) The positive sense of rotation is clockwise about the positive $z$ axis.

There are two parameters to be specified for a coordinate rotation:

1 - Type code 20.0 (signifying a beam coordinate rotation).

2 - The angle of rotation $\alpha$ (degrees).

The angle of rotation may be varied in a first-order fitting (see type code 10.0 ).

Note

This transformation assumes that the units of ( $x$ and $y$ ) and $(\theta$ and $\phi)$ are the same. This is always true unless a 15.03 .0 or a 15.04 .0 type code has been used.

**) See SLAC-75), page 45 and Fig. 4, page 1.2 for definitions of $x, y$, and $z$ coordinates. 
Examples:

For a bending magnet, the beam rotation matrix may be used to specify a rotated magnet.

\section{Excomple No. 1}

A bend up is represented by rotating the $x, y$ coordinates by -90.0 degrees as follows:

20. -90 .

Labels (not to exceed 4 spaces) if desired

2. $B(1)$. ' ;

4. L. B. ก. ' ' ;

2. $\beta(2)$. ', ;

20. +90. ' ' ; (returns coordinates to their initial orientation)

A bend down is accomplished via a +90 degree rotation.

20. +90 . ' ;

2 .

4.

2.

20. -90 . ' ' ;

A bend to the left (looking in the direction of beam travel) is accomplished by rutating the $x$, y coordinates by 180 degrees, e.g. 20. 180. ' ' ;

2.

4.

2.

20. -180 . ' ' ; 
Excomple No. 2

A quadrupole rotated clockwise by 60 degrees about the positive $z$ axis would be specified as follows:

20. 60. ' '

5. L. B. a.' ' ;

20. -60 . ' ' ;

\section{Beam rotation matrix}

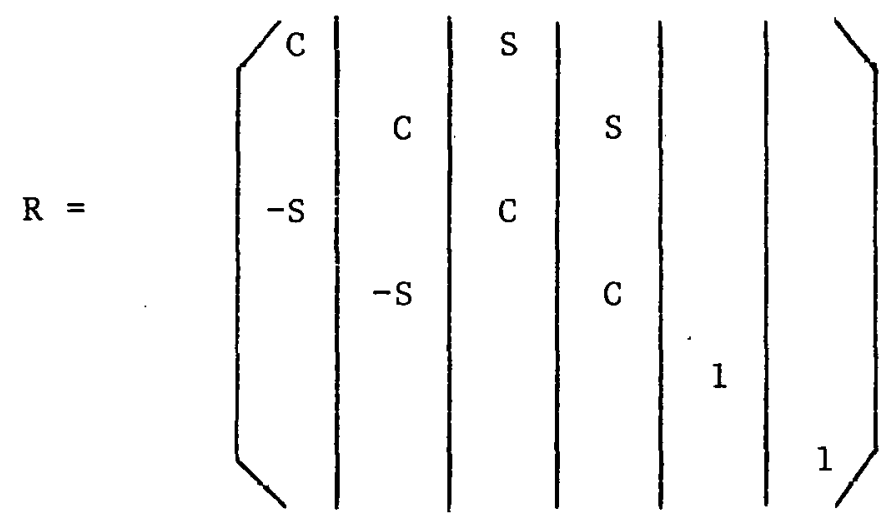

where $\quad C=\cos \alpha$,

$\mathrm{s}=\sin \alpha$,

$\alpha=$ angle of coordinate rotation about the beam axis,

blank spaces are zeros.

e.g. for $\alpha=+90$ degrees, this matrix interchanges rows 1 and 2 with 3 and 4 of the accumulated R matrix as follows:

$\left[\begin{array}{cccc}0 & 0 & 1 & 0 \\ 0 & 0 & 0 & 1 \\ -1 & 0 & 0 & 0 \\ 0 & -1 & 0 & 0\end{array}\right]\left[\begin{array}{llll}K(11) & R(12) & R(13) & R(14) \\ R(21) & R(22)) & R(23) & R(24) \\ R(31) & R(32) & R(33) & R(34) \\ R(41) & R(42) & R(43) & R(44)\end{array}\right]\left[\begin{array}{cccc}R(31) & R(32) & \bar{R}(33) & R(34) \\ R(41) & R(43) & R(43) & R(44) \\ -R(11) & -R(12) & -R(13) & -R(14) \\ -R(21) & -R(22) & -R(23) & -R(24)\end{array}\right]$

(The rest of the matrix is unchanged.) 
STRAY MAGNETIC FIELD: Type code 21.0

1 - Element No. 21.0

2. - Code No. n. $n=4$ : horizontal deflection

$\mathrm{n}=2$ : vertical deflection.

$3-\langle\overline{\mathrm{BL}}\rangle \quad$ mean value of $\int \mathrm{Bd} z$.

$4- \pm\langle\sigma B L\rangle \quad+$ : Gaussian random number generator;

affects beam first moment.

-: uncertainty in $\int \mathrm{Bd} z$ - affects beam second moment.

Uses the misalignment element (8.) to calculate an angular deflection equal to $\int \frac{B d z}{(B \rho)}$

This type code is not functioning in the present version of the prograḿ. 
SENT INEL

Each step of every problem in a TRANSPOR'I data set must be terminated with the word SENTINEL. The word SENTINEL need not be on a separate card. For a description of the form of a TRANSPORT data set see the section on input format.

An entire run, consisting of one or several problems, is indicated by an additional card containing the word SENTINEL. Thus, at the end of the entire data set the word SENTINEL will appear twice.

\section{Acknowledgements}

R. Helm's suggestions and criticisms at SLAC have been invaluable throughout the development of the program and the underlying theory. R. Pordes has ably assisted D. Carey at NAL during the more recent developments of the program. 


\section{REFERENCES}

1. E.D. Courant and H.S. Snyder, Theory of the Alternating Gradient Synchrotron, Ann. Phys. (NY) $\underline{3}, 1-48$ (1958).

2. S. Penner, Calculations of properties of magnetic deflection systems, Rev. Sci. Instrum. 32, 150-160 (1961).

3. K.L. Brown, R. Belbeoch, and P. Bounin, First- and second-order magnetic optics matrix equations for the midplane of uniformfield wedge magnets, Rev. Sci. Instrum. 35, 481-485 (1964).

4. K.L. Brown, A first- and second-order matrix theory for the design of beam transport systems and charged particle spectrometers, SLAC Report No. 75, or Advances Particle Phys. 1, 71-134 (1967).

5. K.L. Brown and S.K. Howry, TRANSPORT/360 a computer program for designing charged particle beam transport systems, SLAC Report No. 91 (1970). The present manual supersedes the above reference.

6. K.L. Brown, A systematic procedure for designing high resolving power beam transport systems or charged particle spectrometers, Proc. 3rd Int. Conf. on Magnet Technology, Hamburg, Germany, May 1970, p. 348, or SLAC-PUB-762 June 1970.

7. H. Enge and S. Kowalski have developed a Ray-Tracing program using essentially the same terminology as TRANSPORT. Any experienced user of TRANSPORT should find it easy to adapt to the M.I.T. program.

\section{SUGGESTED BIBL IOGRAPHY}

A.P. Banford, The transport of charged particle beams (E. and F.N. Spon Ltd., London, 1966).

K.G. Steffen, High-energy beam optics, Interscience Monographs and Texts in Physics and Astronomy, Vol. 17 (John Wiley and Sons, NY, 1965). 
Table 1

Summary of TRANSPORT type codes 
Summary of TRANSPORT type codes.

\begin{tabular}{|c|c|c|c|c|c|c|c|c|c|}
\hline PHYSICAL ELEMENT & $\begin{array}{l}\text { TYPE } \\
\text { CODE }\end{array}$ & $\begin{array}{l}\text { 2nd } \\
\text { ENTRY }\end{array}$ & $\begin{array}{l}3=\mathrm{d} \\
\text { ENTRY }\end{array}$ & $\begin{array}{l}\text { 4th } \\
\text { ENTRY }\end{array}$ & $\begin{array}{l}\text { 5:h } \\
\text { ElNTRY. }\end{array}$ & $\begin{array}{l}\text { 6-h } \\
\text { ElNTRY }\end{array}$ & $\begin{array}{l}\text { 7th } \\
\text { ENTRY }\end{array}$ & $\begin{array}{l}\text { 8th } \\
\text { ENTRY }\end{array}$ & $\begin{array}{l}\text { 9th } \\
\text { ENTRY }\end{array}$ \\
\hline BEAM & $1 . v v r v w 0$ & $x(\mathrm{~cm})$ & $\theta(m)$ & $y(m)$ & $\phi(m r)$ & $\ell(\mathrm{cm})$ & $\delta$ (percent) & $\mathrm{P}_{0}$ & \\
\hline $\begin{array}{l}\text { r.m.s. ADDITION TO } \\
\text { BEAM ENVELOPE }\end{array}$ & 1. wrovroo & $\Delta x(\mathrm{~cm})$ & $\Delta \exists(\mathrm{mr})$ & $\Delta y(\mathrm{~cm})$ & $\Delta \rightarrow(m r)$ & $\Delta 2(\mathrm{~cm})$ & $\Delta \delta($ percent $)$ & $\begin{array}{l}\Delta \mathrm{P} \\
(\mathrm{GeV} / \mathrm{c})\end{array}$ & 0 \\
\hline POLE FACE ROTATION & $2 . \mathrm{v}$ & $\begin{array}{l}\text { ANGLE JF } \\
\text { ROTATIJN (degrees) }\end{array}$ & & r & & & & & \\
\hline DRIFT & $3 . v$ & LENGTH (metres) & & & & & & & \\
\hline BENLING MAGNET & $4 . v v r$ & LENGTH (metres) & FIELD (ki) & $\begin{array}{l}\text { FIELD GRADIENT } \\
\text { (n-value) }\end{array}$ & & & & & \\
\hline QUADRUPOLE & 5. wi] & LENGTH (metres) & FIELDD (KG) & HALF-APERTURE (cm) & & & & & \\
\hline TRANSFORM 1 IJPDATE & 6.0 & 0.0 & & & & & & & \\
\hline TRANSFORM 2 UPDATE & 6.0 & 0.0 & 2.0 & & & & & & \\
\hline BEAM CENTROID SHIFT & 7.0 & SHIFT $(x) ! c m)$ & SIFT $(\epsilon)(\mathrm{mr})$ & $\operatorname{SHIFT}(y)(\mathrm{cm})$ & $9 \mathrm{HIFI}(\phi)(\mathrm{nr})$ & GIFT $(l)(\mathrm{cm})$ & SHIFT ( $\delta$ percent) & & \\
\hline ALIGNMENT TOLERANCE & 8.vrrvvo & DISPLACEMENT $(x)(\mathrm{cm})$ & FOTATION $(\theta)(\mathrm{mr})$ & DISPLACEMENT $(y)(\mathrm{cm})$ & ROTATION $(\phi)(m r)$ & IISPLACEMENT $(z)(\mathrm{cm})$ & ROTATION $(\alpha)(\mathrm{mr})$ & $\begin{array}{l}\text { CODE } \\
\text { NUMBR }\end{array}$ & \\
\hline REPEAT CONTROL & 9.0 & $\begin{array}{l}\text { NUMBEF. GF } \\
\text { REPEATS }\end{array}$ & & 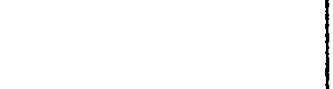 & & & & & \\
\hline FITTING CONSTRAINTS & 10.0 & \pm 1 & $\mathrm{~J}$ & $\begin{array}{l}\text { DESIRED VALUE OF } \\
(\mathrm{I}, \mathrm{J}) \text { MATRIX } \\
\text { ELEMENTS }\end{array}$ & $\begin{array}{l}\text { F.CCURACY :F } \\
\text { EIT }\end{array}$ & & & & \\
\hline \multicolumn{10}{|c|}{$\begin{array}{ll}\text { Note: } & +I \text { is used for fitting a beam }(\sigma) \text { matrix element. } \quad-I \text { is used for fitting an Rl matrix element. } \\
& -(I+20) \text { is used for fitting an Rz metrix e]enent. }\end{array}$} \\
\hline ACCELERATOR & $11 . \mathrm{C}$ & LENGTH (metres) & $\begin{array}{l}\text { E (energy gain! } \\
\text { (GeV) }\end{array}$ & $\begin{array}{l}\phi \text { (phase 1ag) } \\
\text { (degrees) }\end{array}$ & IWAVELENCTH) $(\mathrm{cm})$ & & & & \\
\hline $\begin{array}{l}\text { BEAM } \\
\text { (Rotated Ellipse) }\end{array}$ & 12.0 & THE FIFTEEN COERELET & IONS AMODG THE SIX & MEMTS (This entry mus & ST be preceded by & type code 1.0 entry & & & \\
\hline $\begin{array}{l}\text { INPITT/OUTPUT } \\
\text { OPTIONS }\end{array}$ & 13.0 & $\begin{array}{l}\text { CONTRCL } \\
\text { CODE KURBER }\end{array}$ & & & & & & & \\
\hline ARBITRARY R MATRIX & 14. rovervo & $\mathrm{R}(\mathrm{J}, 1)$ & $R(J, 2)$ & $\mathrm{R}(J, 3)$ & $\mathrm{R}(\mathrm{J}, 4)$ & $\mathbb{R}(J, 5)$ & $R(J, 6)$ & $\mathrm{J}$ & \\
\hline
\end{tabular}




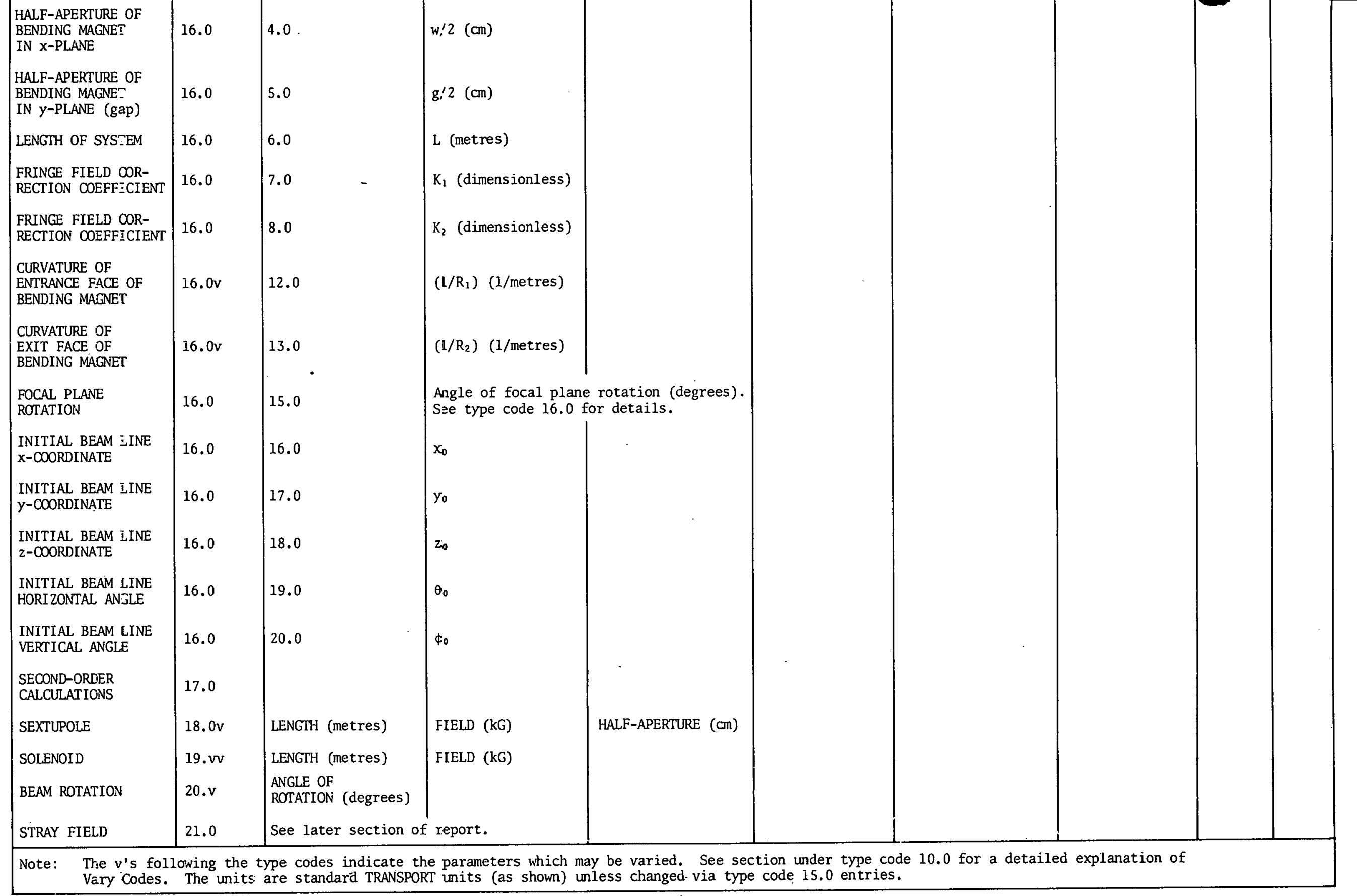

